FIAN-TD-2007-25

arXiv: 0712.3526 [hep-th]

Modified, December 2011

\title{
Cubic interaction vertices for fermionic and bosonic arbitrary spin fields
}

\author{
R.R. Metsaev* \\ Department of Theoretical Physics, P.N. Lebedev Physical Institute, \\ Leninsky prospect 53, Moscow 119991, Russia
}

\begin{abstract}
Using the light-cone gauge approach to relativistic field dynamics, we study arbitrary spin fermionic and bosonic fields propagating in flat space of dimension greater than or equal to four. Generating functions of parity invariant cubic interaction vertices for totally symmetric and mixedsymmetry massive and massless fields are obtained. For the case of totally symmetric fields, we derive restrictions on the allowed values of spins and the number of derivatives. These restrictions provide a complete classification of parity invariant cubic interaction vertices for totally symmetric fermionic and bosonic fields. As an example of application of the light-cone formalism, we obtain simple expressions for the Yang-Mills and gravitational interactions of massive arbitrary spin fermionic fields. For some particular cases, using our light-cone cubic vertices, we discuss the corresponding manifestly Lorentz invariant and on-shell gauge invariant cubic vertices.
\end{abstract}

Keywords: Light-cone approach, interaction vertices, bosonic and fermionic higher-spin fields.

PACS-2011: 11.10.-z; 11.30-j; 11.30.Cp

\footnotetext{
*E-mail: metsaev@1pi.ru
} 


\section{Introduction}

The light-cone gauge approach to relativistic field and string dynamics [1, 2] offers conceptual and technical simplifications into the study of various problems of quantum field and string theories. This approach hides the Lorentz symmetries and makes the notation somewhat cumbersome but eventually turns out to be effective. A number of important problems of modern quantum field and string theories have been solved in the framework of this approach. For example, we mention the solution to the light-cone gauge string field theory [3, 4] and the construction of a superfield formulation for supersymmetric theories (see e.g. Refs.[5]-[8].). Sometimes, theories formulated in the framework of light-cone gauge approach turn out to be a good starting point for deriving a Lorentz covariant formulation (see e.g. Refs.[9]). Use of the light-cone formalism for the building interaction vertices of massless higher-spin fields may be found in Refs.[10]-[14]. Applications of the light-cone formalism for the studying AdS/CFT correspondence are discussed in Refs. [15, 16]. Study of super $p$-branes and string bit models in the light-cone gauge may be found in Refs. [17, [18].

The problem of gravitational interaction of massless higher-spin fields attacked in Ref.[19] was solved in Refs.[20] (for review, see Refs.[21]). This is to say that cubic interaction vertices for totally symmetric massless higher-spin fields in $A d S_{4}$ space were found in Ref.[19], while nonlinear equations of motion to all orders in the coupling constant for totally symmetric massless higherspin fields were obtained in Refs.[20]. At present time it is clear, due to results in Refs. [19, 20], that self-consistent theories of massless higher-spin gauge fields require formulating the theories in $A d S$ background. Recent discussion of interacting massless higher-spin AdS fields theories may be found in Refs.[22].

One of interesting problems of modern research in the theories of higher-spin fields is related to Lagrangian formulation of equations of motions for massless higher-spin fields found in Refs.[20]. In this area of research, some interesting results were recently reported in Ref.[23] (in earlier literature, see Ref.[24]). In order to quantize higher-spin field theories and investigate their ultraviolet behavior, it would be desirable to find an Lagrangian formulation of higher-spin field theories. Since the massless higher-spin field theories involve infinite-dimensional gauge symmetries, one expects that such theories may be ultraviolet finite. We think that the light-cone approach might be helpful in developing light-cone gauge Lagrangian formulation of higher-spin fields theories and understanding their quantum behavior.

In AdS space, the light-cone gauge formulation of free fields dynamics was developed in Ref.[15] (see also Refs.[25, 26]). Because light-cone gauge formulations of fields in AdS and flat spaces share many common properties we believe that methods developed in flat space might be useful and helpful in studying theories of interacting higher-spin AdS fields. In other words, we believe that most of our approach to higher-spin fields in flat space, which we consider in this paper, can straightforwardly be generalized to the case of higher-spin AdS fields.

In Ref.[27], we applied light-cone gauge formalism to the studying cubic interaction vertices of higher-spin massless and massive bosonic fields in flat space. We found generating functions of parity invariant cubic interaction vertices for massive and massless fields of arbitrary symmetry. Also we derive restrictions on the allowed values of spins and the number of derivatives, which provide the complete classification of cubic interaction vertices for totally symmetric massless and massive fields. In earlier literature, application of light-cone formalism for studying interacting higher-spin fields in flat space may be found in Refs.[10]-[14]. In the framework of Lorentz covariant approaches, discussion of interacting higher-spin fields in flat space may be found in Refs.[28] (in earlier literature, see Refs.[29]). For interesting review, see Ref.[30]. 
In this paper, we apply the light-cone formalism for studying cubic interaction vertices for higher-spin bosonic and fermionic fields in flat space. In Ref.[27], we studied cubic interaction vertices involving three bosonic fields, while in the present paper, we study cubic interaction vertices involving two fermionic fields and one bosonic field. In view of possible potentially interesting applications of the higher-spin field theory to superstring theory, it is worthwhile to study cubic vertices for higher-spin fields in flat space of dimension $d \geq 4$. We do this in our paper.

This paper is organized as follows.

In Sec. 2, we discuss the light-cone gauge approach to free fermionic and bosonic fields. We start with the light-cone gauge description of totally symmetric and mixed-symmetry fields in terms of ket-vectors. After this, we review realization of the Poincaré algebra symmetries on space of the ket-vectors.

In Sec. 3, we describe restrictions imposed by the Poincaré algebra symmetries on cubic interaction vertices. We present the complete system of equations for cubic interaction vertices which allows us to determine the vertices uniquely.

In Sec. 4, we discuss cubic vertices for massless fields, i.e., we consider vertices involving two massless fermionic fields and one massless bosonic field. We find generating form for the cubic vertices of massless mixed-symmetry and totally symmetric fields. For the case of totally symmetric fields, we find restrictions on the allowed number of derivatives and spin values of the fields entering the cubic vertices. These restrictions provide the complete classification of the parity invariant cubic vertices for the totally symmetric fields. We use these results to discuss the no-go theorem for the gravitational interaction of higher-spin fermionic fields.

Secs. 5, 10 are devoted to cubic interaction vertices involving both massless and massive fields. As before we discuss the generating form for the cubic vertices of mixed-symmetry and totally symmetric fields. Also, we find restrictions on the allowed number of derivatives and spin values of the totally symmetric fields entering the cubic interaction vertices. We apply our general results to derive the Yang-Mills and gravitational interactions of massive totally symmetric arbitrary spin fermionic fields. Our approach allows us to obtain simple expressions for the cubic vertices of these interactions. We obtain also simple expressions for cubic interaction vertices involving one massless arbitrary spin bosonic field and two massive spin- $\frac{1}{2}$ fermionic fields.

In Sec. 11, we discuss cubic vertices for massive fields: two massive fermionic fields and one massive bosonic field. Generating form of the vertices is obtained for the mixed-symmetry and totally symmetric fields. We derive restrictions on the allowed values of spins and the number of derivatives for cubic vertices of the totally symmetric massive fermionic and bosonic fields.

In Appendix A, we review the notation and conventions used in this paper.

\section{Free light-cone gauge massive and massless fields}

The method proposed in Ref.[1] reduces the problem of finding a new dynamical system to the problem of finding a new solution to commutators of defining symmetry algebra. In our case, the defining symmetries are generated by the Poincare algebra. We begin therefore with a discussion of the realization of the Poincare algebra on the space of massive and massless fields. In this section, we consider free fields.

The Poincaré algebra of $d$-dimensional Minkowski space contains translation generators $P^{A}$ and rotation generators $J^{A B}$ of the Lorentz algebra $s o(d-1,1)$. Non-trivial Poincaré algebra commutators are given by

$$
\left[P^{A}, J^{B C}\right]=\eta^{A B} P^{C}-\eta^{A C} P^{B}, \quad\left[J^{A B}, J^{C E}\right]=\eta^{B C} J^{A E}+3 \text { terms }
$$


where the vector indices of the $s o(d-1,1)$ algebra take values $A, B, C, E=0,1, \ldots, d-1$ and $\eta^{A B}$ stands for the mostly positive flat metric tensor.

To discuss the light-cone approach, in place of the Lorentz frame coordinates $x^{A}$ we introduce the light-cone frame coordinates $x^{+}, x^{-}, x^{I}, I=1, \ldots, d-2$, and treat $x^{+}$as an evolution parameter (for the detailed discussion of the notation, see Appendix A). In the light-cone frame, the Poincaré algebra generators can be separated into two groups:

$$
\begin{array}{llll}
P^{+}, \quad P^{I}, \quad J^{+I}, \quad J^{+-}, & J^{I J}, & \text { kinematical generators } ; \\
P^{-}, \quad J^{-I}, & & \text { dynamical generators }
\end{array}
$$

where the vector indices of the $s o(d-2)$ algebra take values $I, J=1, \ldots, d-2$. For $x^{+}=0$, the kinematical generators in the field realization are quadratic in the physical fields, while the dynamical generators receive higher-order interaction-dependent corrections 1

In light-cone frame, the commutators of the Poincaré algebra can be obtained from (2.1) by using the light-cone metric having the following non vanishing elements: $\eta^{+-}=\eta^{-+}=1, \eta^{I J}=$ $\delta^{I J}$. We adapt the following hermitian conjugation rules for the Poincare algebra generators:

$$
P^{ \pm \dagger}=P^{ \pm}, \quad P^{I \dagger}=P^{I}, \quad J^{I J \dagger}=-J^{I J}, \quad J^{+-\dagger}=-J^{+-}, \quad J^{ \pm I \dagger}=-J^{ \pm I} .
$$

To find a realization of the Poincaré algebra on the space of massive and massless fields we use the light-cone gauge description of those fields.

\subsection{Light-cone gauge massive and massless field}

We now review the light-cone gauge description of free fields propagating in Minkowski space. Throughout the paper a dimension of Minkowski space is assumed to be equal to $d$. An arbitrary spin field of the Poincaré algebra is labelled by mass parameter $m$ and by spin labels $s_{1}, \ldots, s_{\nu}$ for bosonic field and by spin labels $s_{1}+\frac{1}{2}, \ldots, s_{\nu}+\frac{1}{2}$, for fermionic field, where all $s_{n}, n=1, \ldots \nu$, are integers. For massless fields, $\mathrm{m}=0, \nu=\left[\frac{d-2}{2}\right]$, while for massive fields, $\mathrm{m} \neq 0, \nu=\left[\frac{d-1}{2}\right]$. In order to obtain a description of mixed-symmetry fields it is sufficient to set $\nu>1$. In what follows, a particular value of $\nu$ does not matter. We now discuss bosonic and fermionic fields in turn.

Bosonic massless and massive mixed-symmetry fields. In order to streamline the lightcone gauge description of mixed-symmetry fields we introduce a finite set of oscillators $\alpha_{n}^{I}, n=$ $1, \ldots, \nu$, for the discussion of massless fields and a finite set of oscillators $\alpha_{n}^{I}, \zeta_{n}, n=1, \ldots, \nu$, for the discussion of massive fields. Using such set of the oscillators, we introduce the following ket-vectors to discuss the physical D.o.F of mixed-symmetry massless and massive bosonic fields having spin labels $s_{1}, \ldots, s_{\nu}$ :

$$
\begin{array}{rll}
\left|\phi_{s_{1} \ldots s_{\nu}}(p, \alpha)\right\rangle & \equiv \prod_{n=1}^{\nu} \alpha_{n}^{I_{1}^{n}} \ldots \alpha_{n}^{I_{s_{n}}^{n}} \phi_{s_{1} \ldots s_{\nu}}^{I_{1}^{1} \ldots I_{s_{1}}^{1} \ldots I_{1}^{\nu} \ldots I_{s_{\nu}}^{\nu}}(p)|0\rangle, & \text { massless field }, \\
\left|\phi_{s_{1} \ldots s_{\nu}}(p, \alpha)\right\rangle & \equiv \prod_{n=1}^{\nu} \sum_{t_{n}=0}^{s_{n}} \zeta_{n}^{s_{n}-t_{n}} \alpha_{n}^{I_{1}^{n}} \ldots \alpha_{n}^{I_{t_{n}}^{n}} \phi_{s_{1} \ldots s_{\nu}}^{I_{1}^{1} \ldots I_{t_{1}}^{1} \ldots I_{1}^{\nu} \ldots I_{t_{\nu}}^{\nu}}(p)|0\rangle, & \text { massive field. }
\end{array}
$$

In (2.5), (2.6) and below, $\alpha$ occurring in the argument of massless field ket-vector $|\phi(p, \alpha)\rangle$ denotes a set of the oscillators $\left\{\alpha_{n}^{I}\right\}$, while $\alpha$ occurring in the argument of massive field ket-vector $|\phi(p, \alpha)\rangle$

\footnotetext{
${ }^{1}$ For $x^{+} \neq 0$, the kinematical generators take the form $G=G_{1}+x^{+} G_{2}$, where $G_{1}$ is quadratic in fields, while $G_{2}$ contains higher order terms in fields.
} 
denotes a set of the oscillators $\left\{\alpha_{n}^{I}, \zeta_{n}\right\}$. Momentum $p$ occurring in the argument of ket-vector $|\phi(p, \alpha)\rangle$ and $\delta$ - functions denotes a set of the momenta $\left\{p^{I}, \beta \equiv p^{+}\right\}$. Note that we do not explicitly show the dependence of the ket-vector $|\phi(p, \alpha)\rangle$ on the evolution parameter $x^{+}$. Also, note that ket-vector (2.5) is a degree- $s_{n}$ homogeneous polynomial in the oscillators $\alpha_{n}^{I}$, while ketvector (2.6) is a degree- $s_{n}$ homogeneous polynomial in the oscillators $\alpha_{n}^{I}, \zeta_{n}$.

Physical D.o.F of massless and massive fields are described by irreps of the $s o(d-2)$ and $s o(d-1)$ algebras respectively. In order for ket-vectors (2.5) and (2.6) to describe the respective irreps of the $s o(d-2)$ and $s o(d-1)$ algebras we should impose certain algebraic constraints on the ket-vectors. But to avoid unnecessary complications, we impose only the tracelessness constraints,

$$
\begin{array}{lll}
\bar{\alpha}_{m}^{I} \bar{\alpha}_{n}^{I}\left|\phi_{s_{1} \ldots s_{\nu}}(p, \alpha)\right\rangle=0, & m, n=1, \ldots, \nu, & \text { for massless field } \\
\left(\bar{\alpha}_{m}^{I} \bar{\alpha}_{n}^{I}+\bar{\zeta}_{m} \bar{\zeta}_{n}\right)\left|\phi_{s_{1} \ldots s_{\nu}}(p, \alpha)\right\rangle=0, & m, n=1, \ldots, \nu, & \text { for massive field } .
\end{array}
$$

The tracelessness constraints by themselves are not enough to single out irreps of the $s o(d-2)$ and $s o(d-1)$ algebras from the ket-vectors. This implies that ket-vectors (2.5) and (2.6) actually describe finite sets of the respective massless and massive fields.

In what follows, to develop the light-cone gauge description of arbitrary spin mixed-symmetry bosonic fields on an equal footing we use the ket-vector defined by

$$
|\phi(p, \alpha)\rangle \equiv \sum_{s_{1}, \ldots, s_{\nu}=0}^{\infty}\left|\phi_{s_{1} \ldots s_{\nu}}(p, \alpha)\right\rangle .
$$

Totally symmetric massless and massive bosonic fields. Totally symmetric fields are popular in various studies because these fields are considerably simpler than the mixed-symmetry fields and therefore allow to illustrate many important features of higher-spin fields in a relatively straightforward way. In order to obtain a description of massless and massive totally symmetric fields it is sufficient to introduce one sort of oscillators, i.e., we set $\nu=1$ in (2.5) and (2.6) respectively. This is to say that we introduce the following ket-vectors to discuss physical D.o.F. of totally symmetric spin- $s$ massless and massive fields:

$$
\begin{aligned}
& \left|\phi_{s}(p, \alpha)\right\rangle=\alpha^{I_{1}} \ldots \alpha^{I_{s}} \phi^{I_{1} \ldots I_{s}}(p)|0\rangle, \quad \text { massless field } \\
& \left|\phi_{s}(p, \alpha)\right\rangle=\sum_{t=0}^{s} \zeta^{s-t} \alpha^{I_{1}} \ldots \alpha^{I_{t}} \phi^{I_{1} \ldots I_{t}}(p)|0\rangle, \quad \text { massive field. }
\end{aligned}
$$

We note that ket-vector of massless field (2.10) is a degree- $s$ homogeneous polynomial in the oscillator $\alpha^{I}$, while ket-vector of massive field (2.11) is a degree-s homogeneous polynomial in the oscillators $\alpha^{I}, \zeta$,

$$
\begin{array}{ll}
\left(\alpha^{I} \bar{\alpha}^{I}-s\right) \mid \phi_{s}(p, \alpha\rangle=0, & \text { for massless field } \\
\left(\alpha^{I} \bar{\alpha}^{I}+\zeta \bar{\zeta}-s\right)\left|\phi_{s}(p, \alpha)\right\rangle=0, & \text { for massive field }
\end{array}
$$

Physical D.o.F of massless and massive fields are described by irreps of the $s o(d-2)$ and $s o(d-1)$ algebras respectively. In order for ket-vectors (2.10) and (2.11) to realize irreps of the $s o(d-2)$ and $s o(d-1)$ algebras respectively we impose the respective tracelessness constraints

$$
\bar{\alpha}^{I} \bar{\alpha}^{I}\left|\phi_{s}(p, \alpha)\right\rangle=0, \quad \text { for massless field, }
$$




$$
\left(\bar{\alpha}^{I} \bar{\alpha}^{I}+\bar{\zeta}^{2}\right)\left|\phi_{s}(p, \alpha)\right\rangle=0, \quad \text { for massive field }
$$

As in the case of mixed-symmetry fields in order to treat the totally symmetric arbitrary spin bosonic fields on an equal footing we use the ket-vector defined by

$$
|\phi(p, \alpha)\rangle \equiv \sum_{s=0}^{\infty}\left|\phi_{s}(p, \alpha)\right\rangle .
$$

Mixed-symmetry massless and massive fermionic fields. In order to simplify the presentation of light-cone gauge description of mixed-symmetry fermionic fields we use, as before, the finite set of bosonic oscillators $\alpha_{n}^{I}, n=1, \ldots, \nu$, for the discussion of massless fields and the finite set of bosonic oscillators $\alpha_{n}^{I}, \zeta_{n}, n=1, \ldots, \nu$, for the discussion of massive fields. In addition to the bosonic oscillators, we introduce fermionic oscillators denoted by $\theta^{\mathrm{a}}, p_{\theta \mathrm{a}}, \eta^{\mathrm{a}}, p_{\eta \mathrm{a}}$, where subscript and superscript a is used to indicate spinor indices (for the detailed description of the fermionic oscillators, see Appendix A). Using such set of the oscillators, we introduce the following ket-vectors to discuss the physical D.o.F of massless and massive mixed-symmetry fermionic fields having spin labels $s_{1}+\frac{1}{2}, \ldots, s_{\nu}+\frac{1}{2}$ :

$$
\begin{aligned}
& \left|\psi_{s_{1} \ldots s_{\nu}}(p, \alpha)\right\rangle=\left(p_{\theta} \psi_{s_{1} \ldots s_{\nu}}(p, \alpha)+\psi_{s_{1} \ldots s_{\nu}}^{\dagger}(p, \alpha) \eta\right)|0\rangle, \\
& \psi_{s_{1} \ldots s_{\nu}}(p, \alpha) \equiv \prod_{n=1}^{\nu} \alpha_{n}^{I_{1}^{n}} \ldots \alpha_{n}^{I_{s_{n}}^{n}} \psi_{s_{1} \ldots s_{\nu}}^{I_{1}^{1} \ldots I_{s_{1}}^{1} \ldots I_{1}^{\nu} \ldots I_{s_{\nu}}^{\nu}}(p) \\
& \psi_{s_{1} \ldots s_{\nu}}^{\dagger}(p, \alpha) \equiv \prod_{n=1}^{\nu} \alpha_{n}^{I_{1}^{n}} \ldots \alpha_{n}^{I_{s_{n}}^{n}} \psi_{s_{1} \ldots s_{\nu}}^{\dagger I_{1}^{1} \ldots I_{s_{1}}^{1} \ldots I_{1}^{\nu} \ldots I_{s_{\nu}}^{\nu}}(p) \\
& \psi_{s_{1} \ldots s_{\nu}}(p, \alpha) \equiv \prod_{n=1}^{\nu} \sum_{t_{n}=0}^{s_{n}} \zeta_{n}^{s_{n}-t_{n}} \alpha_{n}^{I_{1}^{n}} \ldots \alpha_{n}^{I_{t_{n}}^{n}} \psi_{s_{1} \ldots s_{\nu}}^{I_{1}^{1} \ldots I_{t_{1}}^{1} \ldots I_{1}^{\nu} \ldots I_{t_{\nu}}^{\nu}}(p) \\
& \psi_{s_{1} \ldots s_{\nu}}^{\dagger}(p, \alpha) \equiv \prod_{n=1}^{\nu} \sum_{t_{n}=0}^{s_{n}} \zeta_{n}^{s_{n}-t_{n}} \alpha_{n}^{I_{1}^{n}} \ldots \alpha_{n}^{I_{t_{n}}^{n}} \psi_{s_{1} \ldots s_{\nu}}^{\dagger I_{1}^{1} \ldots I_{t_{1}}^{1} \ldots I_{1}^{\nu} \ldots I_{t_{\nu}}^{\nu}}(p) \text {. }
\end{aligned}
$$

In (2.17)-2.19) and below, the spinor indices of the fermionic oscillators and component fermionic fields are implicit. We note that, for massless field, ket-vector (2.17) is a degree- $s_{n}$ homogeneous polynomial in the oscillator $\alpha_{n}^{I}$, while, for massive field, ket-vector (2.17) is a degree- $s_{n}$ homogeneous polynomial in the oscillators $\alpha_{n}^{I}, \zeta_{n}$.

In order for ket-vector (2.17) to describe the irreps of the $s o(d-2)$ and $s o(d-1)$ algebras we should impose certain algebraic constraints on the ket-vector. But to avoid unnecessary complications, we impose only $\gamma$-tracelessness constraints of $s o(d-2)$ and $s o(d-1)$ algebras,

$$
\begin{aligned}
& \gamma^{I} \bar{\alpha}_{n}^{I} \theta\left|\psi_{s_{1} \ldots s_{\nu}}(p, \alpha)\right\rangle=0, \\
& p_{\eta} \gamma^{I} \bar{\alpha}_{n}^{I}\left|\psi_{s_{1} \ldots s_{\nu}}(p, \alpha)\right\rangle=0 ; \\
& n=1, \ldots, \nu, \quad \text { for massless field } \\
& \left(\gamma^{I} \bar{\alpha}_{n}^{I}+\gamma_{*} \bar{\zeta}_{n}\right) \theta\left|\psi_{s_{1} \ldots s_{\nu}}(p, \alpha)\right\rangle=0 \\
& p_{\eta}\left(\gamma^{I} \bar{\alpha}_{n}^{I}-\gamma_{*} \bar{\zeta}_{n}\right)\left|\psi_{s_{1} \ldots s_{\nu}}(p, \alpha)\right\rangle=0 \text {, } \\
& n=1, \ldots, \nu, \quad \text { for massive field. }
\end{aligned}
$$


This implies that ket-vector (2.17) actually describes finite sets of massless and massive fields.

Throughout this paper, to discuss the light-cone gauge description of arbitrary spin mixedsymmetry fermionic fields an equal footing we use the ket-vector defined by

$$
|\psi(p, \alpha)\rangle \equiv \sum_{s_{1}, \ldots, s_{\nu}=0}^{\infty}\left|\psi_{s_{1} \ldots s_{\nu}}(p, \alpha)\right\rangle
$$

Fermionic massless and massive totally symmetric fields. In order to obtain the light-cone gauge description of a fermionic totally symmetric field we use the bosonic oscillators $\alpha^{I}$ for the discussion of massless field and the bosonic oscillators $\alpha^{I}, \zeta$ for the discussion of massive field. In addition to those bosonic oscillators, we use the fermionic oscillators $\theta^{\mathrm{a}}, p_{\theta \mathrm{a}}, \eta^{\mathrm{a}}, p_{\eta \mathrm{a}}$. Using such oscillators, we collect physical D.o.F of massless and massive totally symmetric spin- $\left(s+\frac{1}{2}\right)$ fermionic fields into the ket-vector defined by

$$
\left|\psi_{s}(p, \alpha)\right\rangle=\left(p_{\theta} \psi_{s}(p, \alpha)+\psi_{s}^{\dagger}(p, \alpha) \eta\right)|0\rangle
$$

where we use the notation

$$
\begin{array}{rlr}
\psi_{s}(p, \alpha) & \equiv \alpha^{I_{1}} \ldots \alpha^{I_{s}} \psi^{I_{1} \ldots I_{s}}(p) \\
\psi_{s}^{\dagger}(p, \alpha) & \equiv \alpha^{I_{1}} \ldots \alpha^{I_{s}} \psi^{\dagger I_{1} \ldots I_{s}}(p) & \\
\psi_{s}(p, \alpha) & \equiv \sum_{t=0}^{s} \zeta^{s-t} \alpha^{I_{1}} \ldots \alpha^{I_{t}} \psi^{I_{1} \ldots I_{t}}(p), & \\
\psi_{s}^{\dagger}(p, \alpha) & \equiv \sum_{t=0}^{s} \zeta^{s-t} \alpha^{I_{1}} \ldots \alpha^{I_{t}} \psi^{\dagger I_{1} \ldots I_{t}}(p), & \text { massless field },
\end{array}
$$

In (2.23)-(2.26) and the subsequent expressions, we do not show the spinor indices of the oscillators and component fermionic fields explicitly. For massless field, ket-vector (2.23) is a degree-s homogeneous polynomial in the oscillator $\alpha^{I}$, while, for massive field, ket-vector (2.23) is degree-s homogeneous polynomial in the oscillators $\alpha^{I}, \zeta$,

$$
\begin{array}{ll}
\left(\alpha^{I} \bar{\alpha}^{I}-s\right)\left|\psi_{s}(p, \alpha)\right\rangle=0, & \text { for massless field } \\
\left(\alpha^{I} \bar{\alpha}^{I}+\zeta \bar{\zeta}-s\right)\left|\psi_{s}(p, \alpha)\right\rangle=0, & \text { for massive field }
\end{array}
$$

We recall that physical D.o.F of massless and massive fields in $d$-dimensional Minkowski space are described by irreps of the respective $s o(d-2)$ and $s o(d-1)$ algebras. For the ket-vector (2.23) to be a carrier of the respective $s o(d-2)$ and $s o(d-1)$ algebra irreps, we impose the following $\gamma$-tracelessness constraints:

$$
\begin{array}{ll}
\gamma^{I} \bar{\alpha}^{I} \theta\left|\psi_{s}(p, \alpha)\right\rangle=0 & \text { for massless field } \\
p_{\eta} \gamma^{I} \bar{\alpha}^{I}\left|\psi_{s}(p, \alpha)\right\rangle=0 & \\
\left(\gamma^{I} \bar{\alpha}^{I}+\gamma_{*} \bar{\zeta}\right) \theta\left|\psi_{s}(p, \alpha)\right\rangle=0, & \text { for massive field } \\
p_{\eta}\left(\gamma^{I} \bar{\alpha}^{I}-\gamma_{*} \bar{\zeta}\right)\left|\psi_{s}(p, \alpha)\right\rangle=0, &
\end{array}
$$


To develop the light-cone gauge description of arbitrary spin totally symmetric fermionic fields on an equal footing we use the ket-vector defined by

$$
|\psi(p, \alpha)\rangle \equiv \sum_{s=0}^{\infty}\left|\psi_{s}(p, \alpha)\right\rangle .
$$

\subsection{Realization of Poincaré algebra symmetries on light-cone gauge fields}

We now discuss a realization of the Poincaré algebra on the space of massless and massive lightcone gauge fields. A representation of kinematical generators (2.2) in terms of differential operators acting on the ket-vectors $|\phi\rangle$ and $|\psi\rangle$ is given by 2

$$
\begin{array}{ll}
P^{I}=p^{I}, & P^{+}=\beta, \\
J^{+I}=\partial_{p^{I}} \beta, & J^{+-}=\partial_{\beta} \beta+M^{+-}, \quad J^{I J}=p^{I} \partial_{p^{J}}-p^{J} \partial_{p^{I}}+M^{I J}, \\
& \beta \equiv p^{+}, \quad \partial_{\beta} \equiv \partial / \partial \beta, \quad \partial_{p^{I}} \equiv \partial / \partial p^{I},
\end{array}
$$

where $M^{+-}$is a spin operator of $s o(1,1)$ algebra, while $M^{I J}$ is a spin operator of the $s o(d-2)$ algebra,

$$
\left[M^{I J}, M^{K L}\right]=\delta^{J K} M^{I L}+3 \text { terms. }
$$

The representation of dynamical generators (2.3) in terms of differential operators acting on the ket-vectors $|\phi\rangle$ and $|\psi\rangle$ is given by

$$
\begin{aligned}
& P^{-}=p^{-}, \quad p^{-} \equiv-\frac{p^{I} p^{I}+\mathrm{m}^{2}}{2 \beta} \\
& J^{-I}=-\partial_{\beta} p^{I}+\partial_{p^{I}} P^{-}+\frac{1}{\beta}\left(M^{I J} p^{J}+\mathrm{m} M^{I}\right)-\frac{p^{I}}{\beta} M^{+-}
\end{aligned}
$$

where $\mathrm{m}$ is the mass parameter and $M^{I}$ is a spin operator transforming in the vector representation of the $s o(d-2)$ algebra. This operator satisfies the commutators

$$
\left[M^{I}, M^{J K}\right]=\delta^{I J} M^{K}-\delta^{I K} M^{J}, \quad\left[M^{I}, M^{J}\right]=-M^{I J} .
$$

From (2.34), (2.37), we see that the operators $M^{I J}$ and $M^{I}$ satisfy commutators of the $s o(d-1)$ algebra. The particular form of the operators $M^{+-}, M^{I J}$, and $M^{I}$ depends on the choice of the realization of spin D.o.F of physical fields. We now present realization of these operators on space of the ket-vectors $|\phi\rangle$ and $|\psi\rangle$ discussed in Section 2.1 (see (2.9), (2.16), (2.22), (2.30)). Realization of the spin operator $M^{+-}$on space of the ket-vectors $|\phi\rangle$ and $|\psi\rangle$ is given by

$$
\begin{array}{ll}
M^{+-}=0, & \text { for bosonic fields } \\
M^{+-}=-\frac{1}{2} p_{\theta} \theta-\frac{1}{2} \eta p_{\eta}, & \text { for fermionic fields. }
\end{array}
$$

\footnotetext{
${ }^{2}$ In this paper, without loss of generality, we analyze the Poincare algebra generators and their commutators for $x^{+}=0$.
} 
Realization of the operators $M^{I J}$ and $M^{I}$ on space of the ket-vectors $|\phi\rangle$ and $|\psi\rangle$ corresponding to the bosonic and fermionic mixed-symmetry massive fields is given by

$$
\begin{array}{ll}
M^{I J}=\sum_{n=0}^{\nu} \alpha_{n}^{I} \bar{\alpha}_{n}^{J}-\alpha_{n}^{J} \bar{\alpha}_{n}^{I}, & \text { for mix.-symm. boson. field, } \\
M^{I}=\sum_{n=0}^{\nu} \zeta_{n} \bar{\alpha}_{n}^{I}-\alpha_{n}^{I} \bar{\zeta}_{n}, & \\
M^{I J}=\sum_{n=0}^{\nu} \alpha_{n}^{I} \bar{\alpha}_{n}^{J}-\alpha_{n}^{J} \bar{\alpha}_{n}^{I}+\frac{1}{2} p_{\theta} \gamma^{I J} \theta+\frac{1}{2} p_{\eta} \gamma^{I J} \eta, & \\
M^{I}=\sum_{n=0}^{\nu} \zeta_{n} \bar{\alpha}_{n}^{I}-\alpha_{n}^{I} \bar{\zeta}_{n}-\frac{1}{2} p_{\theta} \gamma^{I} \gamma_{*} \theta+\frac{1}{2} p_{\eta} \gamma^{I} \gamma_{*} \eta, &
\end{array}
$$

Realization of the operators $M^{I J}$ and $M^{I}$ on space of the ket-vectors $|\phi\rangle$ and $|\psi\rangle$ corresponding to the bosonic and fermionic totally symmetric massive fields takes the form

$$
\begin{array}{ll}
M^{I J}=\alpha^{I} \bar{\alpha}^{J}-\alpha^{J} \bar{\alpha}^{I}, & \text { for mix.-symm. boson. field, } \\
M^{I}=\zeta \bar{\alpha}^{I}-\alpha^{I} \bar{\zeta}, & \\
M^{I J}=\alpha^{I} \bar{\alpha}^{J}-\alpha^{J} \bar{\alpha}^{I}+\frac{1}{2} p_{\theta} \gamma^{I J} \theta+\frac{1}{2} p_{\eta} \gamma^{I J} \eta, & \text { for mix.-symm. ferm. field. } \\
M^{I}=\zeta \bar{\alpha}^{I}-\alpha^{I} \bar{\zeta}-\frac{1}{2} p_{\theta} \gamma^{I} \gamma_{*} \theta+\frac{1}{2} p_{\eta} \gamma^{I} \gamma_{*} \eta, &
\end{array}
$$

From (2.36), we see that in the massless limit, $m \rightarrow 0$, the generators of the Poincare algebra are independent of the operator $M^{I}$. This implies that the free light-cone gauge dynamics of massive fields have a smooth limit to the free light-cone gauge dynamics of massless fields.

The above expressions provide a realization of the Poincaré algebra in terms of differential operators acting on the physical fields collected into the ket-vectors $|\phi\rangle$ and $|\psi\rangle$. We now write a field theoretical realization of this algebra in terms of the ket-vectors $|\phi\rangle,|\psi\rangle$. As mentioned above the kinematical generators $G^{\text {kin }}$ are realized quadratically in the ket-vectors $|\phi\rangle$, $|\psi\rangle$, while the dynamical generators $G^{\text {dyn }}$ are realized non-linearly. At the quadratic level, both $G^{\text {kin }}$ and $G^{\text {dyn }}$ admit the representation

$$
\begin{aligned}
G_{[2]}=G_{[2]}^{\mathrm{bos}} & +G_{[2]}^{\mathrm{fer}}, \\
G_{[2]}^{\mathrm{bos}} & =\int d^{d-1} p\langle\phi(p)|\beta G| \phi(p)\rangle, \\
G_{[2]}^{\mathrm{fer}} & =\int d^{d-1} p\langle\psi(p)|G| \psi(p)\rangle,
\end{aligned}
$$

$d^{d-1} p \equiv d \beta d^{d-2} p$, where $G$ are the differential operators given in (2.31), (2.32), (2.35), (2.36) and the notation $G_{[2]}$ is used for the field theoretical free generators. In (2.45), (2.46) and below, the bra-vectors are defined as $\langle\phi(p)| \equiv(|\phi(p)\rangle)^{\dagger},\langle\psi(p)| \equiv(|\psi(p)\rangle)^{\dagger}$. The bosonic ket-vector $|\phi\rangle$ and fermionic ket-vector $|\psi\rangle$ satisfy the Poisson-Dirac commutators

$$
\left.\left[|\phi(p, \alpha)\rangle,\left|\phi\left(p^{\prime}, \alpha^{\prime}\right)\right\rangle\right]\right|_{\text {equal } x^{+}}=\frac{1}{2 \beta} \delta^{d-1}\left(p+p^{\prime}\right)|\rangle|\rangle^{\prime},
$$




$$
\left.\left[|\psi(p, \alpha)\rangle,\left|\psi\left(p^{\prime}, \alpha^{\prime}\right)\right\rangle\right]\right|_{\text {equal } x^{+}}=\frac{1}{2} \delta^{d-1}\left(p+p^{\prime}\right)|\rangle|\rangle^{\prime},
$$

where |\rangle|\rangle$^{\prime}$ is defined by

$$
|\rangle|\rangle^{\prime}= \begin{cases}\Pi|0\rangle\left|0^{\prime}\right\rangle & \text { for bosonic fields } \\ \left(p_{\theta} \eta^{\prime}+\eta p_{\theta}^{\prime}\right) \Pi|0\rangle\left|0^{\prime}\right\rangle & \text { for fermionic fields }\end{cases}
$$

and $\Pi$ is a unity operator on a space of ket-vectors given in (2.5),(2.6). Note that, in terms of component fermionic fields (2.18),(2.19), the commutator in (2.48) is realized as the anti-commutator. With these definitions, we have the standard commutators

$$
\begin{aligned}
& {\left.\left[|\phi\rangle, G_{[2]}\right]\right|_{{\text {equal } x^{+}}}=G|\phi\rangle,} \\
& {\left.\left[|\psi\rangle, G_{[2]}\right]\right|_{{\text {equal } x^{+}}}=G|\psi\rangle .}
\end{aligned}
$$

In the framework of the Lagrangian approach, the light-cone gauge action takes the standard form

$$
S=\int d x^{+} d^{d-1} p\left(\left\langle\phi(p)\left|\mathrm{i} \beta \partial^{-}\right| \phi(p)\right\rangle+\left\langle\psi(p)\left|\mathrm{i} \partial^{-}\right| \psi(p)\right\rangle\right)+\int d x^{+} P^{-},
$$

where $P^{-}$is the light-cone frame Hamiltonian. Representation for the light-cone action given in (2.52) is valid for the free and for the interacting theory. The Hamiltonian of free theory can be obtained by using relations (2.35), (2.44).

The internal symmetry can be incorporated into the theory under consideration by adopting the Chan-Paton method in string theory [31] (see e.g. Ref. [32]).

\section{Equations for cubic interaction vertices}

We now discuss the Poincaré algebra dynamical generators given in (2.3). In theories of interacting fields, the dynamical generators of the Poincaé algebra receive corrections containing higher powers of physical fields. The dynamical generators can be expanded as

$$
G^{\mathrm{dyn}}=\sum_{n=2}^{\infty} G_{[n]}^{\mathrm{dyn}}
$$

where $G_{[n]}^{\mathrm{dyn}}$ stands for the functional that has $n$ powers of physical fields. 3 In what follows, the $G_{[2]}^{\mathrm{dyn}}$ and $G_{[3]}^{\mathrm{dyn}}$ contributions appearing in expansion (3.1) will be referred to as the respective quadratic and cubic dynamical generators. The quadratic dynamical generators for massless and massive mixed-symmetry fields were discussed in Sec.2. Our purpose is to find cubic dynamical generators $G_{[3]}^{\mathrm{dyn}}$ for the massless and massive mixed-symmetry fields. The cubic dynamical generators can be found by using

i) restrictions imposed by kinematical symmetries;

ii) dynamical light-cone principle.

The detailed treatment of restrictions imposed by the kinematical symmetries and the dynamical light-cone principle may be found in Sec.3 in Ref.[27]. Therefore to avoid the repetition we briefly outline our procedure of the derivation of the cubic dynamical generators.

\footnotetext{
${ }^{3}$ The dynamical generators of supersymmetric Yang-Mills theories do not receive corrections of the order higher than four in fields (see e.g. Refs.[5]), while the dynamical generators of (super)gravity theories are nontrivial for all $n \geq 2$ (see e.g. Refs.[33]). Note also that the generators of the closed string field theories, which involve the graviton field, terminate at cubic correction $G_{[3]}^{\mathrm{dyn}}[4]$. This implies that, in string theory, the general covariance is realized in a somewhat nontrivial way. Interesting discussion of this theme may be found in Ref. [34].
} 


\subsection{Restrictions imposed by kinematical symmetries}

In this section, we discuss the restrictions imposed by kinematical symmetries on the cubic dynamical generators $P_{[3]}^{-}$and $J_{[3]}^{-I}$. Namely, we describe those properties of the cubic dynamical generators that can be obtained from commutators between $P^{-}, J^{-I}$ and the kinematical generators given in (2.2). This is to say that, using commutators between kinematical generators (2.2) and the dynamical generators $P^{-}, J^{-I}$, we find the following representation for the cubic dynamical generators:

$$
\begin{aligned}
& P_{[3]}^{-}=\int d \Gamma_{3}\left\langle\Phi_{[3]} \mid p_{[3]}^{-}\right\rangle, \\
& J_{[3]}^{-I}=\int d \Gamma_{3}\left(\left\langle\Phi_{[3]} \mid j_{[3]}^{-I}\right\rangle-\frac{1}{3}\left(\sum_{a=1}^{3} \partial_{p_{a}^{I}}\left\langle\Phi_{[3]}\right|\right)\left|p_{[3]}^{-}\right\rangle\right),
\end{aligned}
$$

where we use the notation

$$
\begin{aligned}
\left|p_{[3]}^{-}\right\rangle & \equiv p_{[3]}^{-} \prod_{a=1}^{3}|0\rangle_{a}, \quad\left|j_{[3]}^{-I}\right\rangle \equiv j_{[3]}^{-I} \prod_{a=1}^{3}|0\rangle_{a}, \\
\left\langle\Phi_{[3]}\right| & \equiv\left\langle\psi\left(p_{1}, \alpha_{1}\right)\right|\left\langle\psi\left(p_{2}, \alpha_{2}\right)\right|\left\langle\phi\left(p_{3}, \alpha_{3}\right)\right|, \\
d \Gamma_{3} & \equiv(2 \pi)^{d-1} \delta^{d-1}\left(\sum_{a=1}^{3} p_{a}\right) \prod_{a=1}^{3} \frac{d^{d-1} p_{a}}{(2 \pi)^{(d-1) / 2}} .
\end{aligned}
$$

Here and below, the indices $a, b=1,2,3$ label three interacting fields involved in cubic vertices.

For generosity, we assume that the bra-vectors $\left\langle\psi\left(p_{1}, \alpha_{1}\right)\right|,\left\langle\psi\left(p_{2}, \alpha_{2}\right)\right|$ describe two fermionic fields having different mass values and internal charges. Note that, in general, the generators $P_{[3]}^{-}$ (3.2) are not hermitian. Hermitian $P_{[3]}^{-}$can be obtained in two obvious ways. The first hermitian representative of $P_{[3]}^{-}$is given by $P_{[3]}^{-}+$h.c., while the second hermitian representative of $P_{[3]}^{-}$is given by $1 P_{[3]}^{-}+$h.c.. So, in general, there are two families of hermitian generators $P_{[3]}^{-}$. At the end of Section 11.1.1, we present some examples of those two families of hermitian vertices.

Densities $p_{[3]}^{-}, j_{[3]}^{-I}$ in (3.2), (3.3) depend on the momenta $\mathbb{P}^{I}, \beta_{a}, a=1,2,3$, and variables related to the spin D.o.F, which we denote by $\alpha$ :

$$
\begin{aligned}
p_{[3]}^{-}= & p_{[3]}^{-}\left(\mathbb{P}, \beta_{a} ; \alpha\right), \\
j_{[3]}^{-I}= & j_{[3]}^{-I}\left(\mathbb{P}, \beta_{a} ; \alpha\right), \\
& \mathbb{P}^{I} \equiv \frac{1}{3} \sum_{a=1}^{3} \check{\beta}_{a} p_{a}^{I}, \quad \check{\beta}_{a} \equiv \beta_{a+1}-\beta_{a+2}, \quad \beta_{a} \equiv \beta_{a+3} .
\end{aligned}
$$

We note also that the densities $p_{[3]}^{-}, j_{[3]}^{-I}$ should satisfy the following equations

$$
\begin{aligned}
& \mathbf{J}^{I J}\left|p_{[3]}^{-}\right\rangle=0, \\
& \left(\mathbb{P}^{I} \partial_{\mathbb{P}^{I}}+\sum_{a=1}^{3} \beta_{a} \partial_{\beta_{a}}-\mathbf{M}^{+-\dagger}\right)\left|p_{[3]}^{-}\right\rangle=0, \\
& \mathbf{J}^{I J}\left|j_{[3]}^{-K}\right\rangle+\delta^{I K}\left|j_{[3]}^{-J}\right\rangle-\delta^{J K}\left|j_{[3]}^{-I}\right\rangle=0,
\end{aligned}
$$




$$
\left(\mathbb{P}^{I} \partial_{\mathbb{P} I}+\sum_{a=1}^{3} \beta_{a} \partial_{\beta_{a}}-\mathbf{M}^{+-\dagger}\right)\left|j_{[3]}^{-K}\right\rangle=0
$$

where we use the notation

$$
\begin{array}{rrl}
\mathbf{J}^{I J} \equiv \mathbf{L}^{I J}+\mathbf{M}^{I J}, & \mathbf{L}^{I J} \equiv \mathbb{P}^{I} \partial_{\mathbb{P}^{J}}-\mathbb{P}^{J} \partial_{\mathbb{P}^{I}} \\
\mathbf{M}^{I J} \equiv \sum_{a=1}^{3} M^{(a) I J}, & \mathbf{M}^{+-\dagger} \equiv \sum_{a=1}^{3} M^{(a)+-\dagger}
\end{array}
$$

From (3.2), (3.3), we see that the problem of finding the cubic generators $P_{[3]}^{-}, J_{[3]}^{-I}$ is reduced to the problem of finding the respective densities $p_{[3]}^{-}, j_{[3]}^{-I}$ subject to kinematical symmetry restrictions given in (3.10)-(3.13). The restrictions given in (3.10)-(3.13) by themselves are not enough to determine the densities $p_{[3]}^{-}, j_{[3]}^{-I}$ uniquely. We proceed therefore to the discussion of the light-cone dynamical principle.

\subsection{Restrictions imposed by light-cone dynamical principle}

To determine the densities $p_{[3]}^{-}, j_{[3]}^{-I}$ uniquely we use method which we refer to as the light-cone dynamical principle. The light-cone dynamical principle is summarized as the following three steps:

Step 1. Using commutators of the Poincaré algebra for the dynamical generators $P^{-}$and $J^{-I}$, we find restrictions imposed on the densities $p_{[3]}^{-}, j_{[3]}^{-I}$. At this step, we make sure that the density $j_{[3]}^{-I}$ can be expressed in terms of the density $p_{[3]}^{-}$.

Step 2. We require the densities $p_{[3]}^{-}, j_{[3]}^{-I}$ to be polynomials in the momentum $\mathbb{P}^{I}$. We refer to this requirement as the light-cone locality condition.

Step 3. We find the density $p_{[3]}^{-}$that cannot be removed by field redefinitions.

We now proceed with discussing the restrictions imposed by the light-cone dynamical principle on the densities $p_{[3]}^{-}, j_{[3]}^{-I}$. In what follows, the density $p_{[3]}^{-}$will be referred to as cubic interaction vertex. We now discuss the three steps of our method in turn.

Step 1. At this step, we find the restrictions imposed by the Poincare algebra commutators between the dynamical generators. All that is required is to consider the commutators

$$
\begin{aligned}
& {\left[P^{-}, J^{-I}\right]=0,} \\
& {\left[J^{-I}, J^{-J}\right]=0 .}
\end{aligned}
$$

In the cubic approximation, commutators (3.16) lead to the equation for the densities $\left|p_{[3]}^{-}\right\rangle,\left|j_{[3]}^{-I}\right\rangle$ given by

$$
\mathbf{P}^{-}\left|j_{[3]}^{-I}\right\rangle=-\mathbf{J}^{-I \dagger}\left|p_{[3]}^{-}\right\rangle,
$$

where $\mathbf{P}^{-}, \mathbf{J}^{-I \dagger}$ are defined as

$$
\begin{aligned}
& \mathbf{P}^{-} \equiv \frac{\mathbb{P}^{I} \mathbb{P}^{I}}{2 \hat{\beta}}-\sum_{a=1}^{3} \frac{\mathrm{m}_{a}^{2}}{2 \beta_{a}} \\
& \mathbf{J}^{-I \dagger} \equiv-\frac{1}{3 \hat{\beta}} \mathcal{X}^{I},
\end{aligned}
$$




$$
\begin{aligned}
& \mathcal{X}^{I} \equiv X^{I J} \mathbb{P}^{J}+X^{I}+X \partial_{\mathbb{P}^{I}} \\
& X^{I J} \equiv \sum_{a=1}^{3} \check{\beta}_{a}\left(\left(\beta_{a} \partial_{\beta_{a}}-M^{(a)+-\dagger}\right) \delta^{I J}-M^{(a) I J}\right) \\
& X^{I} \equiv \sum_{a=1}^{3} \frac{3 \hat{\beta} \mathrm{m}_{a}}{\beta_{a}} M^{(a) I} \\
& X \equiv-\sum_{a=1}^{3} \frac{\hat{\beta} \check{\beta}_{a} \mathrm{~m}_{a}^{2}}{2 \beta_{a}} \\
& \hat{\beta} \equiv \beta_{1} \beta_{2} \beta_{3} .
\end{aligned}
$$

Taking (3.20) into account, we can rewrite Eq.(3.18) as

$$
\left|j_{[3]}^{-I}\right\rangle=\frac{1}{3 \hat{\beta} \mathbf{P}^{-}} \mathcal{X}^{I}\left|p_{[3]}^{-}\right\rangle,
$$

i.e., as we have promised, the density $j_{[3]}^{-I}$ is expressed in terms of vertex $p_{[3]}^{-}$(3.7). Plugging $j_{[3]}^{-I}$ (3.26) into (3.17), we make sure that, in the cubic approximation, commutators (3.17) are satisfied automatically. Thus, we see that commutators (3.16), (3.17) amount to relation (3.26).

Before proceeding to the second step of our method, we note that plugging $j_{[3]}^{-I}(3.26)$ into Eqs.(3.12), (3.13), we make sure that these equations are satisfied provided the density $p_{[3]}^{-}$is satisfied Eqs.(3.10), (3.11). Also, we note that, we exhausted all commutators of the Poincaré algebra in the cubic approximation. Equations for cubic vertex (3.10), (3.11) and relation (3.26) provide the complete list of restrictions imposed by commutators of the Poincare algebra on the densities $p_{[3]}^{-}, j_{[3]}^{-I}$. We see that the restrictions imposed by commutators of the Poincaré algebra by themselves are not sufficient to determine the cubic interaction vertex $p_{[3]}^{-}$uniquely. To find the cubic interaction vertex $p_{[3]}^{-}$we proceed to the next steps of our method.

Step 2. At this step, we impose the light-cone locality condition: we require the densities $p_{[3]}^{-}$, $j_{[3]}^{-I}$ to be polynomials in the momentum $\mathbb{P}^{I}$. As regards the vertex $p_{[3]}^{-}$, we require this vertex to be local (i.e. polynomial in $\mathbb{P}^{I}$ ) from the very beginning. However it is clear from relation (3.26) that a local $p_{[3]}^{-}$does not lead automatically to a local density $j_{[3]}^{-I}$. From (3.26), we see that the light-cone locality condition for $j_{[3]}^{-I}$ amounts to the equation

$$
\mathcal{X}^{I}\left|p_{[3]}^{-}\right\rangle=\mathbf{P}^{-}\left|V^{I}\right\rangle
$$

where a vertex $\left|V^{I}\right\rangle$ is restricted to be polynomial in $\mathbb{P}^{I}$.

Step 3. The last requirement we impose on the vertex $p_{[3]}^{-}$is motivated by the desire to deal with vertex that cannot be removed by field redefinitions. Using local (i.e. polynomial in the transverse momentum $\mathbb{P}^{I}$ ) field redefinitions, we can remove in the cubic vertex $p_{[3]}^{-}$those terms that are proportional to $\mathbf{P}^{-}$(see Appendix B in Ref.[27].). Since we are interested in the cubic vertex $p_{[3]}^{-}$that cannot be removed by field redefinitions, we impose the following restriction:

$$
\left|p_{[3]}^{-}\right\rangle \neq \mathbf{P}^{-}|V\rangle
$$

where $\mathbf{P}^{-}$is given in (3.19), and a vertex $|V\rangle$ is restricted to be polynomial $\mathbb{P}^{I}$.

Altogether, equations (3.26)-(3.28) exhaust restrictions imposed by the light-cone dynamical principle. 
Complete system of equations for cubic interaction vertex. We now collect equations imposed by the kinematical symmetries and the light-cone dynamical principle on vertex $p_{[3]}^{-}(3.7)$ :

$$
\begin{array}{ll}
\mathbf{J}^{I J}\left|p_{[3]}^{-}\right\rangle=0, & \text { so }(d-2) \text { invariance, } \\
\left(\mathbb{P}^{I} \partial_{\mathbb{P}^{I}}+\sum_{a=1}^{3} \beta_{a} \partial_{\beta_{a}}-\mathbf{M}^{+-\dagger}\right)\left|p_{[3]}^{-}\right\rangle=0, & \text { so }(1,1) \text { invariance, } \\
\mathcal{X}^{I}\left|p_{[3]}^{-}\right\rangle=\mathbf{P}^{-}\left|V^{I}\right\rangle, & \\
\left|p_{[3]}^{-}\right\rangle \neq \mathbf{P}^{-}|V\rangle, & \text { light-cone dynamical principle. } \\
\left|j_{[3]}^{-I}\right\rangle=\frac{1}{3 \hat{\beta}}\left|V^{I}\right\rangle, & \\
\left|p_{[3]}^{-}\right\rangle,|V\rangle,\left|V^{I}\right\rangle \text { are polynomials in } \mathbb{P}^{I}, &
\end{array}
$$

Equations (3.29), (3.30) reflect the invariance of the vertex $\left|p_{[3]}^{-}\right\rangle$under the respective $s o(d-2)$ and $s o(1,1)$ rotations. Equations given in (3.31) summarize the restrictions obtained by using the light-cone dynamical principle.

To summarize, equations (3.29)-(3.31) constitute a complete system of equations on vertex $p_{[3]}^{-}$ (3.7). These equations allow us to determine the cubic vertex uniquely (up to coupling constants).

General structure of cubic vertices in Lorentz covariant and light-cone gauge approaches. Manifestly Lorentz invariant vertices are beyond the scope of this paper. However for the reader convenience and in order to introduce some terminology we discuss general structure of covariant vertices which are related to light-cone gauge vertices we study in this paper. Let $\Psi=\Psi(x)$ be a set of fermionic arbitrary spin Dirac fields of the Lorentz algebra, while $\Phi=\Phi(x)$ is a set of arbitrary spin bosonic fields of the Lorentz algebra, where the argument $x$ stands for space-time coordinates. Let $\gamma$ be Dirac $\gamma$-matrices, while $\partial$ stands for derivatives w.r.t the space-time coordinates. In general, vertices for massless and massive fields involve contributions with different powers of $\partial$. Therefore, to classify cubic vertices we need two labels at least. We can use labels $k_{\min }^{\text {cov }}$ and $k_{\max }^{\text {cov }}$ which are the respective minimal and maximal numbers of $\partial$ appearing in a manifestly Lorentz invariant vertex. Using this notation we note that, in Lorentz covariant approach, on-shell cubic vertices involving two fermionic fields and one bosonic field can schematically be presented as power series expansion in $\partial$,

$$
\mathcal{L}^{\mathrm{cov}}=\bar{\Psi} M_{\max } \partial^{k_{\max }^{\mathrm{cov}}} \Psi \Phi+\ldots+\bar{\Psi} M_{\min } \partial^{k_{\min }^{\mathrm{cov}}} \Psi \Phi
$$

where matrices $M_{\max }$ and $M_{\min }$ are constructed out of flat metric tensor and Dirac $\gamma$-matrices. Let us restrict our attention to vertices that do not involve Levi-Civita antisymmetric tensor and matrix $\Gamma_{*}$ (for definition the matrix $\Gamma_{*}$, see Appendix A). We shall refer to such vertices as parity invariant vertices. For the parity invariant vertices, the matrix $M_{\max }$ involves either even number of gamma-matrices or odd number of gamma-matrices. Using this, we separate vertices into two groups: K-vertices and F-vertices. This is to say that if matrix $M_{\max }$ in (3.32) involves even number of $\gamma$-matrices then the vertex is referred to as $\mathrm{K}$-vertex, while, if matrix $M_{\max }$ in (3.32) involves odd number of $\gamma$-matrices then the vertex is referred to as F-vertex. For example, using the nomenclature of $\mathrm{K}$ - and F-vertices, we note that the covariant vertex $\bar{\psi} \psi \phi$ is referred to as $\mathrm{K}$-vertex, while the covariant vertex describing electro-magnetic interaction $\bar{\psi} \gamma^{A} \psi \phi^{A}$ is referred to as F-vertex in this paper. 
In this paper, we restrict our attention to the light-cone vertices corresponding to the parity invariant cubic vertices (3.32). Such light-cone gauge cubic vertices can also be presented in the form similar to one in (3.32),

$$
\mathcal{L}^{\text {l.c. }}=\bar{\psi} M_{\max }^{\text {l.c. }} \partial^{k_{\text {max }}^{1 . c .}} \psi \phi+\ldots+\bar{\psi} M_{\min }^{\text {l.c. }} \partial^{k_{\min }^{1 . c .}} \psi \phi
$$

where light-cone frame matrices $M_{\max }^{\text {l.c. }}$ and $M_{\min }^{\text {l.c. }}$ are constructed out of the flat delta-Kronecker of the $s o(d-2)$ algebra and light-cone frame Dirac $\gamma$-matrices. To classify light-cone vertices we also use nomenclature of $\mathrm{K}$-vertices and F-vertices. This is to say the light-cone counterparts of the covariant K- and F-vertices will also be refereed to as K- and F-vertices respectively. Note however that, for the case of light-cone $\mathrm{K}$-vertex in (3.33), the matrix $M_{\text {max }}^{\text {l.c. involves odd number }}$ of $\gamma$-matrices, while, for the case of light-cone F-vertex in (3.33), the matrix $M_{\max }^{\text {l.c. }}$ involves even number of $\gamma$-matrices.

For the examples of on-shell manifestly Lorentz invariant vertices considered in this paper and their light-cone counterparts, we find the following relation:

$$
k_{\max }^{\mathrm{cov}}=k_{\max }^{\text {l.c. }}-1 \text {. }
$$

We note also that for vertices involving two massless fermionic and one massless bosonic fields one has the relations $k_{\max }^{\mathrm{cov}}=k_{\min }^{\mathrm{cov}}, k_{\max }^{\text {l.c. }}=k_{\min }^{\text {l.c. }}$. Taking this into account, we introduce the notation $k^{\text {cov }}$ for a number of derivatives appearing in on-shell Lorentz invariant vertices of massless fields and notation $k^{\text {l.c. }}$ for a number of transverse derivatives appearing in light-cone gauge vertices of massless fields to obtain the following relation 4

$$
k^{\mathrm{cov}}=k^{\text {l.c. }}-1, \quad \text { for massless fields. }
$$

Throughout this paper we drop the superscript l.c. and use the following simplified notation:

$$
k \equiv k^{\text {l.c. }}, \quad k_{\max } \equiv k_{\max }^{\text {l.c. }}, \quad k_{\min } \equiv k_{\min }^{\text {l.c. }} .
$$

We now turn to equations for cubic vertices in (3.29)-3.31). Up to this point our treatment has been applied to vertices for massive as well as massless fields. From now on, we separately consider vertices involving only massless fields, vertices involving both massless and massive fields, and vertices involving only massive fields. Depending on the values of mass parameters of fields entering cubic vertices, we can separately consider cubic vertices with the following mass values:

$$
\begin{aligned}
& \left.\mathrm{m}_{1}\right|_{F}=0,\left.\quad \mathrm{~m}_{2}\right|_{F}=0,\left.\quad \mathrm{~m}_{3}\right|_{B}=0 ; \\
& \left.\mathrm{m}_{1}\right|_{F}=\left.\mathrm{m}_{2}\right|_{F}=0,\left.\quad \mathrm{~m}_{3}\right|_{B} \neq 0 ; \\
& \left.\mathrm{m}_{1}\right|_{B}=\left.\mathrm{m}_{2}\right|_{F}=0,\left.\quad \mathrm{~m}_{3}\right|_{F} \neq 0 ; \\
& \left.\mathrm{m}_{1}\right|_{F}=\left.\mathrm{m}_{2}\right|_{F} \equiv \mathrm{m} \neq 0,\left.\quad \mathrm{~m}_{3}\right|_{B}=0 ; \\
& \left.\mathrm{m}_{1}\right|_{B}=\left.\mathrm{m}_{2}\right|_{F} \equiv \mathrm{m} \neq 0,\left.\quad \mathrm{~m}_{3}\right|_{F}=0 ; \\
& \left.\mathrm{m}_{1}\right|_{F} \neq 0,\left.\quad \mathrm{~m}_{2}\right|_{F} \neq 0,\left.\quad \mathrm{~m}_{1}\right|_{F} \neq\left.\mathrm{m}_{2}\right|_{F},\left.\quad \mathrm{~m}_{3}\right|_{B}=0 ;
\end{aligned}
$$

\footnotetext{
${ }^{4}$ For the case of vertices involving only massless bosonic fields, one has the relation $k^{\text {cov }}=k^{\text {l.c. }}$.
} 


$$
\begin{aligned}
& \left.\mathrm{m}_{1}\right|_{B} \neq 0,\left.\quad \mathrm{~m}_{2}\right|_{F} \neq 0,\left.\quad \mathrm{~m}_{1}\right|_{B} \neq\left.\mathrm{m}_{2}\right|_{F},\left.\quad \mathrm{~m}_{3}\right|_{F}=0 ; \\
& \left.\mathrm{m}_{1}\right|_{F} \neq 0,\left.\quad \mathrm{~m}_{2}\right|_{F} \neq 0,\left.\quad \mathrm{~m}_{3}\right|_{B} \neq 0 .
\end{aligned}
$$

The notation $\left.\mathrm{m}_{a}\right|_{B}$ implies that $\mathrm{m}_{a}$ is a mass parameter of bosonic field carrying the external line index $a$, while the notation $\left.\mathrm{m}_{b}\right|_{F}$ implies that $\mathrm{m}_{b}$ is a mass parameter of fermionic field carrying the external line index $b$. Vertices with mass parameters in (3.37) describe interaction of massless fields, while the ones in (3.44) describe interaction of massive fields. Vertices with mass parameters given in (3.38), (3.39) describe interaction of two massless fields with one massive field, while the vertices in (3.40) - (3.43) describe interaction of two massive fields with one massless field.

We now present solutions to defining equations (3.29)-(3.31) for cubic vertices with mass parameters given in (3.37)-(3.44) in turn. The solutions can be found by using the same methods we used to analyze cubic vertices for bosonic fields in Ref.[27] (see Section 4.2 and Appendix D in Ref.[27]). Therefore to avoid the repetitions we just present the results for cubic vertices and discuss their properties.

\section{Cubic vertices for massless fermionic and bosonic fields}

In this section, we discuss the cubic interaction vertex for the massless mixed-symmetry fields (3.37). We consider vertex involving two massless mixed-symmetry fermionic fields having the mass parameters $\mathrm{m}_{1}=0$ and $\mathrm{m}_{2}=0$ and one massless mixed-symmetry bosonic field having the mass parameter $\mathrm{m}_{3}=0$ :

$$
\left.\mathrm{m}_{1}\right|_{F}=0,\left.\quad \mathrm{~m}_{2}\right|_{F}=0,\left.\quad \mathrm{~m}_{3}\right|_{B}=0 .
$$

Equations for the vertex involving three massless fields are obtainable from (3.31) by letting $\mathrm{m}_{a} \rightarrow$ $0, a=1,2,3$. We find the following two types of solutions for cubic vertices:

$$
\begin{array}{ll}
p_{[3]}^{-}=K^{(12)} V^{\mathrm{K}}\left(B_{n}^{(a)} ; Z_{m n q}\right), & \text { K-vertex; } \\
p_{[3]}^{-}=F_{n} V^{\mathrm{F}}\left(B_{n}^{(a)} ; Z_{m n q}\right), & \text { F-vertex } ;
\end{array}
$$

where $V^{\mathrm{K}}, V^{\mathrm{F}}$ are arbitrary polynomials of $B_{n}^{(a)}$ and $Z_{m n q}$ and we use the notation

$$
\begin{aligned}
& K^{(12)}=\frac{1}{\beta_{1} \beta_{2}} p_{\theta_{1}} \mathbb{P}^{I} \gamma^{I} \gamma_{*} \eta_{2}, \\
& F_{n}=\frac{1}{\beta_{1} \beta_{2}} p_{\theta_{1}}\left(\frac{\check{\beta}_{3}}{\beta_{3}} \mathbb{P}^{I}-\gamma^{I J} \mathbb{P}^{J}\right) \eta_{2} \alpha_{n}^{(3) I}, \\
& B_{n}^{(a)} \equiv \frac{\alpha_{n}^{(a) I} \mathbb{P}^{I}}{\beta_{a}}, \quad a=1,2,3, \\
& Z_{m n q} \equiv B_{m}^{(1)} \alpha_{n q}^{(23)}+B_{n}^{(2)} \alpha_{q m}^{(31)}+B_{q}^{(3)} \alpha_{m n}^{(12)}, \\
& \alpha_{m n}^{(a b)} \equiv \alpha_{m}^{(a) I} \alpha_{n}^{(b) I} .
\end{aligned}
$$


We recall that, for the mixed-symmetry fields, the subindices $m, n, q$ take the following values:

$$
m, n, q=1, \ldots, \nu,
$$

where $\nu$ is arbitrary integer $\nu>1$. Note that the quantities $B_{n}^{(a)}, \alpha_{m n}^{(a b)}$ and $Z_{m n q}$ are the respective degree-1, 2, and 3 homogeneous polynomials in the oscillators. In what follows, we refer to degree-1, 2, and 3 homogeneous polynomials in the oscillators as linear, quadratic, and cubic forms respectively.

We now make comments on the solution obtained.

i) We see that solution for vertices in (4.2), (4.3) is governed by the prefactors $K^{(12)}, F_{n}$, and the generating functions $V^{\mathrm{K}}, V^{\mathrm{F}}$. The generating functions are arbitrary polynomials of the linear forms $B_{n}^{(a)}$ and the cubic forms $Z_{m n q}$.

ii) Equations for vertices allow solutions for the generating functions $V^{\mathrm{K}}, V^{\mathrm{F}}$ that depend on the quadratic forms $\alpha_{m n}^{(11)}, \alpha_{m n}^{(22)}, \alpha_{m n}^{(33)}$. However, in view of tracelessness constraint (see (2.7), (2.20)), such quadratic forms do not contribute to the Hamiltonian. Therefore we drop dependence on the quadratic forms $\alpha_{m n}^{(a a)}$ in the generating functions $V^{\mathrm{K}}, V^{\mathrm{F}}$.

iii) The prefactors, the linear forms $B_{n}^{(a)}$, and cubic forms $Z_{m n q}$ are homogeneous polynomials in momentum $\mathbb{P}^{I}$. Appearance of the prefactors, linear forms and cubic forms that are homogeneous polynomials in $\mathbb{P}^{I}$ is characteristic feature of vertices for massless fields.

iv) We note that the prefactor $K^{(12)}$ involves only odd number of transverse $\gamma$-matric, while the prefactors $F_{n}$ involve only even number of the transverse $\gamma$-matrices.

v) Solution for the generating functions $V^{\mathrm{K}}, V^{\mathrm{F}}$ given in (4.2), (4.3) is complete solution, while the solution for the prefactors $K^{(12)}, F_{n}$ given in (4.4), (4.5) is not complete solution. This is to say that for the vertices of mixed-symmetry fields there are extra solutions for the prefactors that involve contributions of higher than second order in $\gamma$-matrices. We note also for the vertices of totally symmetric fields those extra solutions are trivial.

To understand the remaining interesting properties of the vertices we consider cubic vertices for totally symmetric massless fields.

\subsection{Cubic vertices for massless totally symmetric fields}

We now consider the parity invariant cubic interaction vertices for massless totally symmetric fields. To this end it is sufficient to use one sort of oscillators, i.e. to set $\nu=1$ in (4.9). To simplify our presentation of cubic vertices we drop oscillator's subscript $n=1$ and use the simplified notation $\alpha^{I} \equiv \alpha_{1}^{I}$. Then the cubic vertex is obtained from solution given in (4.2), (4.3) by using the identifications

$$
\alpha^{(a) I} \equiv \alpha_{1}^{(a) I}, \quad a=1,2,3,
$$

and ignoring contribution of oscillators carrying a subscript $n>1$. Adopting notation (4.10) for prefactor $F \equiv F_{1}$, linear forms $B^{(a)} \equiv B_{1}^{(a)}(4.6)$, quadratic forms $\alpha^{(a b)} \equiv \alpha_{11}^{(a b)}(4.8)$, and cubic form $Z \equiv Z_{111}$ (4.7), we get

$$
\begin{array}{ll}
p_{[3]}^{-}=K^{(12)} V^{\mathrm{K}}\left(B^{(a)} ; Z\right), & \text { K-vertex; } \\
p_{[3]}^{-}=F V^{\mathrm{F}}\left(B^{(a)} ; Z\right), & \text { F-vertex; }
\end{array}
$$

where $V^{\mathrm{K}}, V^{\mathrm{F}}$ are arbitrary polynomials of $B^{(a)}$ and $Z$ and we use the notation

$$
K^{(12)}=\frac{1}{\beta_{1} \beta_{2}} p_{\theta_{1}} \mathbb{P}^{I} \gamma^{I} \gamma_{*} \eta_{2}
$$




$$
\begin{gathered}
F=\frac{1}{\beta_{1} \beta_{2}} p_{\theta_{1}}\left(\frac{\check{\beta}_{3}}{\beta_{3}} \mathbb{P}^{I}-\gamma^{I J} \mathbb{P}^{J}\right) \eta_{2} \alpha^{(3) I}, \\
B^{(a)} \equiv \frac{\alpha^{(a) I} \mathbb{P}^{I}}{\beta_{a}}, \quad a=1,2,3, \\
Z \equiv B^{(1)} \alpha^{(23)}+B^{(2)} \alpha^{(31)}+B^{(3)} \alpha^{(12)} \\
\alpha^{(a b)} \equiv \alpha^{(a) I} \alpha^{(b) I} .
\end{gathered}
$$

We now make comment on the solution obtained.

i) Solution for vertices in (4.11), (4.12) is governed by prefactors $K^{(12)}, F$ and by generating functions $V^{\mathrm{K}}, V^{\mathrm{F}}$. The generating functions are arbitrary polynomials of the three linear forms $B^{(a)}$ and the one cubic form $Z$. As compared to the case for mixed-symmetry fields, the number of linear forms and cubic forms is decreased and this simplifies considerably the study of the solution. ii) Equations for vertices allow solutions for $V^{\mathrm{K}}, V^{\mathrm{F}}$ that depend on the quadratic forms $\alpha^{(11)}, \alpha^{(22)}$, $\alpha^{(33)}$ (4.17). However, due to traceless constraint (see (2.14), (2.28) ), such quadratic forms do not contribute to the Hamiltonian. Therefore we drop dependence on the quadratic forms $\alpha^{(a a)}$ in the generating functions $V^{\mathrm{K}}, V^{\mathrm{F}}$.

iii) Solution for the generating functions $V^{\mathrm{K}}, V^{\mathrm{F}}$ given in (4.11), (4.12) is complete solution. We note that, in contrast to the vertices for mixed-symmetry fields, solution for the prefactors given in (4.13), (4.14) is also complete solution.

\subsubsection{Cubic vertices for totally symmetric fields with fixed but arbitrary spin values}

Vertices (4.11), (4.12) describe interaction of towers of massless totally symmetric bosonic and fermionic fields (2.16), (2.30). We now consider vertices involving two massless totally symmetric spin $s^{(1)}+\frac{1}{2}$ and $s^{(2)}+\frac{1}{2}$ fermionic fields and one massless totally symmetric spin- $s^{(3)}$ bosonic field:

$$
\begin{array}{lll}
\mathrm{m}_{1}=0, & \mathrm{~m}_{2}=0, & \mathrm{~m}_{3}=0, \\
s^{(1)}+\frac{1}{2}, & s^{(2)}+\frac{1}{2}, & s^{(3)} .
\end{array}
$$

The massless spin $s^{(1)}+\frac{1}{2}$ and $s^{(2)}+\frac{1}{2}$ fermionic fields are described by the respective ket-vectors $\left|\psi_{s^{(1)}}\right\rangle$ and $\left|\psi_{s^{(2)}}\right\rangle$, while the massless spin- $s^{(3)}$ bosonic field is described by the ket-vector $\left|\phi_{s^{(3)}}\right\rangle$. The ket-vectors of massless fermionic fields are obtainable from (2.23) by replacement $s \rightarrow s^{(a)}$, $\alpha^{I} \rightarrow \alpha^{(a) I}, a=1,2$, in (2.23), while the ket-vector of massless bosonic field is obtainable from (2.10) by replacement $s \rightarrow s^{(3)}, \alpha^{I} \rightarrow \alpha^{(3) I}$ in (2.10). Taking into account that the ket-vectors for massless fields $\left|\psi_{s^{(1)}}\right\rangle,\left|\psi_{s^{(2)}}\right\rangle,\left|\phi_{s^{(3)}}\right\rangle$ are the respective degree- $s^{(a)}, a=1,2,3$, homogeneous polynomials in the oscillators $\alpha^{(a) I}$, it is easy to see that the vertices we are interested in must satisfy the equations

$$
\left(\alpha^{(a) I} \bar{\alpha}^{(a) I}-s^{(a)}\right)\left|p_{[3]}^{-}\right\rangle=0, \quad a=1,2,3,
$$

which tell us that the vertices should be degree- $s^{(a)}$ homogeneous polynomials in the oscillators $\alpha^{(a) I}$. Taking into account that the prefactor $F$ and linear forms $B^{(a)}$ are degree- 1 homogeneous polynomials in the oscillators, while the cubic form $Z$ is the degree- 3 homogeneous polynomial in the oscillators (see (4.14)-(4.16)), we find the general solution of Eqs.(4.19),

$$
p_{[3]}^{-}\left(s^{(1)}+\frac{1}{2}, s^{(2)}+\frac{1}{2}, s^{(3)} ; k\right)=K^{(12)} Z^{\frac{1}{2}(\mathbf{s}-k+1)} \prod_{a=1}^{3}\left(B^{(a)}\right)^{s^{(a)}+\frac{1}{2}(k-\mathbf{s}-1)}, \quad \text { for K-vertex; }
$$




$$
\begin{gathered}
p_{[3]}^{-}\left(s^{(1)}+\frac{1}{2}, s^{(2)}+\frac{1}{2}, s^{(3)} ; k\right)=F Z^{\frac{1}{2}(\mathbf{s}-k)}\left(B^{(3)}\right)^{-1} \prod_{a=1}^{3}\left(B^{(a)}\right)^{s^{(a)}+\frac{1}{2}(k-\mathbf{s})}, \quad \text { for F-vertex; } \\
\mathbf{s} \equiv \sum_{a=1}^{3} s^{(a)}
\end{gathered}
$$

where integer $k$ is a freedom in our solution. The integer $k$ labels all possible cubic vertices that can be built for massless spin $s^{(1)}+\frac{1}{2}, s^{(2)}+\frac{1}{2}, s^{(3)}$ fields and has a clear physical interpretation. Taking into account that the prefactors $K^{(12)}, F$, and the forms $B^{(a)}, Z$ are degree-1 homogeneous polynomials in the momentum $\mathbb{P}^{I}$, it is easy to see that vertices (4.20), (4.21) are degree- $k$ homogeneous polynomials in $\mathbb{P}^{I}$. To summarize, the vertex $p_{[3]}^{-}\left(s^{(1)}+\frac{1}{2}, s^{(2)}+\frac{1}{2}, s^{(3)} ; k\right)$ describes interaction of two massless fermionic spin $s^{(1)}+\frac{1}{2}, s^{(2)}+\frac{1}{2}$ fields and one bosonic massless spin- $s^{(3)}$ field and the vertex is degree- $k$ homogeneous polynomial in the momentum $\mathbb{P}^{I}$. In Lorentz covariant approach, gauge invariant vertices corresponding to our light-cone vertices (4.20), (4.21) contain $k-1$ number of the derivatives with respect to space-time coordinates.

Restrictions on spin values and number of derivatives in cubic vertices. We now discuss the restrictions to be imposed on spin values $s^{(1)}, s^{(2)}, s^{(3)}$ and the integer $k$. The powers of the forms $B^{(a)}$ and $Z$ in (4.20), (4.21) must be non-negative integers. For this to be the case, it is necessary to impose the following restrictions on the allowed spin values $s^{(1)}, s^{(2)}, s^{(3)}$ and the number of powers of the momentum $\mathbb{P}^{I}$ (the number of the transverse derivatives):

$$
\begin{array}{ll}
\mathbf{s}-2 s_{\min }+1 \leq k \leq \mathbf{s}+1, \quad s_{\min } \equiv \min \left(s^{(1)}, s^{(2)}, s^{(3)}\right), \\
\mathbf{s}-k \text { odd integer, } & \text { for K-vertex } \\
\mathbf{s}-2 \min \left(s^{(1)}, s^{(2)}, s^{(3)}-1\right) \leq k \leq \mathbf{s}, & \\
\mathbf{s}-k \text { even integer, } & \text { for F-vertex . } \\
s^{(3)} \geq 1 \quad \text { when } \quad s^{(3)}=s_{\min }, &
\end{array}
$$

We now consider K- and F- vertices for the space-time dimensions $d>4$ and $d=4$ in turn.

Case $d>4$. Restrictions (4.23), (4.24) lead to a surprisingly simple result for values of allowed $k$ for cubic vertices $p_{[3]}^{-}\left(s^{(1)}+\frac{1}{2}, s^{(2)}+\frac{1}{2}, s^{(3)} ; k\right)$. Indeed, we see from (4.23) and (4.24) that for spin values $s^{(1)}, s^{(2)}, s^{(3)}$, the integer $k$ takes the values

$$
\begin{array}{ll}
k=\mathbf{s}+1, \mathbf{s}-1, \ldots, \mathbf{s}-2 s_{\min }+1, & \text { for K-vertices in } d>4 \\
k=\mathbf{s}, \mathbf{s}-2, \ldots, \mathbf{s}-2 \min \left(s^{(1)}, s^{(2)}, s^{(3)}-1\right), & \text { for F-vertices in } d>4 .
\end{array}
$$

Note that, for F-vertices, we should keep in mind the restriction $s^{(3)} \geq 1$ when $s^{(3)}=s_{\text {min }}$. Vertices (4.20), (4.21), with $k$ in (4.25),(4.26), constitute the complete list of parity invariant cubic vertices for $d>4$. Relations (4.25), (4.26) imply that given spin values $s^{(1)}, s^{(2)}, s^{(3)}$, the numbers of parity invariant cubic interaction vertices that can be constructed are given by

$$
\begin{array}{ll}
\mathrm{N}=s_{\min }+1, & \text { for K-vertices in } d>4 \\
\mathrm{~N}=\min \left(s^{(1)}, s^{(2)}, s^{(3)}-1\right)+1, & \text { for F-vertices in } d>4
\end{array}
$$


Case $d=4$. For $d=4$, the number of allowed vertices is decreased. This is, if $d=4$, then for spin values $s^{(1)}, s^{(2)}, s^{(3)}$, the integer $k$ takes the values 5

$$
\begin{array}{lll}
k=\mathbf{s}+1, \mathbf{s}-2 s_{\min }+1, & \text { when } s^{(3)}=s_{\min } & \text { for K-vertices in } d=4 . \\
k=\mathbf{s}+1, & \text { when } s^{(3)}>s_{\min }, & \text { for K-vertices in } d=4 ; \\
k=\mathbf{s}-2 s_{\min }, & \text { when } s^{(3)}>s_{\text {min }}, & \text { for F-vertices in } d=4 .
\end{array}
$$

This implies that, in $4 d$, there are two parity invariant $\mathrm{K}$-vertices when $s^{(3)}=s_{\min }>0$ and one parity invariant K-vertex when $s^{(3)}=s_{\min }=0$ or $s^{(3)}>s_{\min }$. Also we note that, in $4 d$, there is only one parity invariant F-vertex when $s^{(3)}>s_{\min }$ and there are no parity invariant F-vertices when $s^{(3)}=s_{\text {min }}$.

Formulas (4.20), (4.21) provide a surprisingly simple form for the vertices of massless higherspin fields and also give a simple form for the vertices of the well-known massless low-spin fields having spin values $0, \frac{1}{2}, 1, \frac{3}{2}, 2$. By way of example, we consider light-cone gauge cubic vertices and their Lorentz covariant counterparts that describe interaction of massless spin $-\frac{1}{2},-\frac{3}{2}$ fermionic fields with massless low-spin and higher-spin bosonic fields.

i) Cubic vertex for massless spin- $\frac{1}{2}$ fermionic field $\psi$ and massless scalar field $\phi$ takes the form

$$
p_{[3]}^{-}\left(\frac{1}{2}, \frac{1}{2}, 0 ; 1\right)=K^{(12)} \sim \bar{\psi} \psi \phi .
$$

ii) The minimal interaction of massless spin- $\frac{1}{2}$ fermionic field $\psi$ with a massless spin- 1 bosonic field $\phi^{A}$ is given by

$$
p_{[3]}^{-}\left(\frac{1}{2}, \frac{1}{2}, 1 ; 1\right)=F \sim \bar{\psi} \gamma^{A} \psi \phi^{A},
$$

iii) The non-minimal interaction of massless spin- $\frac{1}{2}$ fermionic field with massless spin- 1 bosonic field takes the form

$$
p_{[3]}^{-}\left(\frac{1}{2}, \frac{1}{2}, 1 ; 2\right)=K^{(12)} B^{(3)} \sim \bar{\psi} \gamma^{A B} \psi F^{A B}, \quad F^{A B} \equiv \partial^{A} \phi^{B}-\partial^{B} \phi^{A} .
$$

iv) The gravitational interaction of massless spin- $\frac{1}{2}$ fermionic field $\psi$ is given by

$$
p_{[3]}^{-}\left(\frac{1}{2}, \frac{1}{2}, 2 ; 2\right)=\left.F B^{(3)} \sim \bar{\psi} \gamma^{A} D^{A} \psi\right|_{[3]},
$$

where $D^{A}$ stands for covariant derivative w.r.t. Lorentz connection and the subscript [3] of covariant Lagrangian is used to indicate the cubic vertex.

v) The non-minimal interaction of massless spin- $\frac{1}{2}$ fermionic field $\psi$ with massless spin- 2 bosonic field $\phi^{A B}$ takes the form

$$
p_{[3]}^{-}\left(\frac{1}{2}, \frac{1}{2}, 2 ; 3\right)=K^{(12)}\left(B^{(3)}\right)^{2} \sim \bar{\psi} \partial^{A} \partial^{B} \psi \phi^{A B} .
$$

vi) Our approach gives simple expressions for cubic vertices of massless spin- $\frac{1}{2}$ fermionic field and massless spin-s bosonic field. Namely, there are the following two cubic vertices for massless spin- $\frac{1}{2}$ fermionic field $\psi$ and massless totally symmetric arbitrary spin- $s$ bosonic field $\phi^{A_{1} \ldots A_{s}}$ :

$$
p_{[3]}^{-}\left(\frac{1}{2}, \frac{1}{2}, s ; s+1\right)=K^{(12)}\left(B^{(3)}\right)^{s} \sim \bar{\psi} \partial^{A_{1}} \ldots \partial^{A_{s}} \psi \phi^{A_{1} \ldots A_{s}},
$$

${ }^{5}$ There is the simple rule which allows to choose nontrivial vertices in $4 d$. This, an appearance of $K^{(12)} Z B^{(3)}-$ factor in K-vertex leads to trivial K-vertex, while appearance of $F B^{(1)} B^{(2)}$-factor in F-vertex leads to trivial F-vertex. This rule can be approved by using helicity formalism in Ref.[10]. 


$$
p_{[3]}^{-}\left(\frac{1}{2}, \frac{1}{2}, s ; s\right)=F\left(B^{(3)}\right)^{s-1} \sim \bar{\psi} \gamma^{A_{1}} \partial^{A_{2}} \ldots \partial^{A_{s}} \psi \phi^{A_{1} \ldots A_{s}}
$$

vii) Higher-derivative interaction of massless spin- $\frac{3}{2}$ fermionic field $\psi^{A}$ (gravitino field) with massless scalar field $\phi$ takes the form

$$
p_{[3]}^{-}\left(\frac{3}{2}, \frac{3}{2}, 0 ; 3\right)=K^{(12)} B^{(1)} B^{(2)} \sim \bar{\Psi}^{A B} \Psi^{A B} \phi, \quad \Psi^{A B} \equiv \partial^{A} \psi^{B}-\partial^{B} \psi^{A},
$$

where $\Psi^{A B}$ is field strength of the spin- $\frac{3}{2}$ fermionic field $\psi^{A}$.

viii) The non-minimal interaction vertices of massless spin- $\frac{3}{2}$ fermionic field $\psi^{A}$ with massless spin-1 bosonic field $\phi^{A}$ having the field strength $F^{A B}$ as in (4.34) take the form

$$
\begin{aligned}
& p_{[3]}^{-}\left(\frac{3}{2}, \frac{3}{2}, 1 ; 2\right)=K^{(12)} Z \sim\left(\bar{\psi}^{A} \psi^{B}-\frac{1}{4} \psi^{C} \gamma^{A B} \psi^{C}\right) F^{A B}, \\
& p_{[3]}^{-}\left(\frac{3}{2}, \frac{3}{2}, 1 ; 3\right)=F B^{(1)} B^{(2)} \sim \bar{\psi}^{A} \partial^{A} \gamma^{B} \psi^{C} F^{B C}, \\
& p_{[3]}^{-}\left(\frac{3}{2}, \frac{3}{2}, 1 ; 4\right)=K^{(12)} B^{(1)} B^{(2)} B^{(3)} \sim \bar{\psi}^{A} \partial^{A} \partial^{B} \psi^{C} F^{B C} .
\end{aligned}
$$

ix) The minimal gravitational interaction of massless spin- $\frac{3}{2}$ fermionic field $\psi^{A}$ is given by

$$
p_{[3]}^{-}\left(\frac{3}{2}, \frac{3}{2}, 2 ; 2\right)=\left.F Z \sim \bar{\psi}^{A} \gamma^{A B C} D_{\text {Lor }}^{B} \psi^{C}\right|_{[3]} \text {. }
$$

x) Light-cone gauge cubic vertices for two massless spin- $\frac{3}{2}$ fermionic fields and one massless arbitrary spin- $s, s \geq 2$, bosonic field take the form

$$
\begin{aligned}
& p_{[3]}^{-}\left(\frac{3}{2}, \frac{3}{2}, s ; s\right)=F Z\left(B^{(3)}\right)^{s-2}, \\
& p_{[3]}^{-}\left(\frac{3}{2}, \frac{3}{2}, s ; s+1\right)=K^{(12)} Z\left(B^{(3)}\right)^{s-1}, \\
& p_{[3]}^{-}\left(\frac{3}{2}, \frac{3}{2}, s ; s+2\right)=F B^{(1)} B^{(2)}\left(B^{(3)}\right)^{s-1}, \\
& p_{[3]}^{-}\left(\frac{3}{2}, \frac{3}{2}, s ; s+3\right)=K^{(12)} B^{(1)} B^{(2)}\left(B^{(3)}\right)^{s} .
\end{aligned}
$$

Using the notation $\mathcal{L}\left(\frac{3}{2}, \frac{3}{2}, s ; k\right)$ for Lorentz covariant vertices corresponding to the respective lightcone gauge vertices $p_{[3]}^{-}\left(\frac{3}{2}, \frac{3}{2}, s ; k\right)$ in (4.44)-(4.47), we obtain

$$
\begin{aligned}
\mathrm{i}^{s-1} \mathcal{L}\left(\frac{3}{2}, \frac{3}{2}, s ; s\right)=\bar{\psi}^{A_{1}} \gamma^{A_{2}} \partial^{A_{3}} \ldots \partial^{A_{s}} \psi^{B} \partial^{B} \phi^{A_{1} \ldots A_{s}} & \\
& +\bar{\psi}^{C} \gamma^{A_{1}} \partial^{A_{2}} \ldots \partial^{A_{s-1}} \Psi^{C A_{s}} \phi^{A_{1} \ldots A_{s}}, \\
\mathrm{i}^{s-1} \mathcal{L}\left(\frac{3}{2}, \frac{3}{2}, s ; s+1\right)= & \bar{\psi}^{A_{1}} \partial^{A_{2}} \ldots \partial^{A_{s}} \psi^{B} \partial^{B} \phi^{A_{1} \ldots A_{s}} \\
& +\bar{\psi}^{C} \partial^{A_{1}} \ldots \partial^{A_{s-1}} \Psi^{C A_{s}} \phi^{A_{1} \ldots A_{s}}, \\
\mathrm{i}^{s-1} \mathcal{L}\left(\frac{3}{2}, \frac{3}{2}, s ; s+2\right)= & \bar{\psi}^{B} \gamma^{A_{1}} \partial^{A_{2}} \ldots \partial^{A_{s}} \psi^{C} \partial^{B} \partial^{C} \phi^{A_{1} \ldots A_{s}},
\end{aligned}
$$




$$
\mathrm{i}^{s-1} \mathcal{L}\left(\frac{3}{2}, \frac{3}{2}, s ; s+3\right)=\bar{\psi}^{B} \partial^{A_{1}} \ldots \partial^{A_{s}} \psi^{C} \partial^{B} \partial^{C} \phi^{A_{1} \ldots A_{s}}
$$

where $\phi^{A_{1} \ldots A_{s}}$ stands for massless totally symmetric spin-s bosonic field. We note that covariant vertices in (4.48)-(4.51) are gauge invariant only under linearized on-shell gauge symmetries. Also, note that, for $s=2$, the vertex in (4.48) is nothing but the vertex of the minimal gravitational interaction of massless spin- $\frac{3}{2}$ fermionic field (4.43).

Thus, we see that the light-cone gauge approach does indeed provide a simple representation for interaction vertices. Another attractive property of the light-cone approach is that it allows treating the interaction vertices on an equal footing. Formulas (4.20), (4.21) provide a convenient representation for other well-known cubic interaction vertices of massless low-spin fields. These vertices and their Lorentz covariant counterparts are collected in Table I.

Table I. Cubic vertices for massless fermionic and bosonic low-spin fields. In the 4th column, $\phi, \psi, \phi^{A}, \psi^{A}, \phi^{A B}$ stand for the respective spin $0,1 / 2,1,3 / 2,2$ massless fields. $F^{A B}$ and $R^{A B C E}$ stand for the respective Yang-Mills field strength and the Riemann tensor, while $\Psi^{A B}=\partial^{A} \psi^{B}-\partial^{B} \psi^{A}$ is field strength of spin 3/2 field $\psi^{A} . D^{A}$ and $D_{L o r}^{A}$ stand for the respective Yang-Mills covariant derivative and Lorentz covariant derivative. $\omega_{A}{ }^{B C}$ stands for the linearized Lorentz connection, $\omega_{A}{ }^{B C}=-\omega_{A}{ }^{B C}$. Most of the covariant vertices in the Table are invariant only under linearized on-shell gauge transformations.

\begin{tabular}{|c|c|c|c|}
\hline $\begin{array}{c}\text { Spin values and } \\
\text { number of derivatives } \\
s^{(1)}+\frac{1}{2}, s^{(2)}+\frac{1}{2}, s^{(3)} ; k\end{array}$ & $\begin{array}{l}\text { dimension } \\
\text { of } \\
\text { space-time }\end{array}$ & $\begin{array}{c}\text { Light-cone vertex } \\
p_{[3]}^{-}\left(s^{(1)}+\frac{1}{2}, s^{(2)}+\frac{1}{2}, s^{(3)} ; k\right)\end{array}$ & $\begin{array}{l}\text { Manifestly Lorentz } \\
\text { invariant vertex }\end{array}$ \\
\hline$\frac{1}{2}, \frac{1}{2}, 0 ; 1$ & $d \geq 4$ & $K^{(12)}$ & $\bar{\psi} \psi \phi$ \\
\hline$\frac{1}{2}, \frac{1}{2}, 1 ; 1$ & $d \geq 4$ & $F$ & $\bar{\psi} \gamma^{A} \psi \phi^{A}$ \\
\hline$\frac{1}{2}, \frac{1}{2}, 1 ; 2$ & $d \geq 4$ & $K^{(12)} B^{(3)}$ & $\bar{\psi} \gamma^{A B} \psi F^{A B}$ \\
\hline$\frac{1}{2}, \frac{1}{2}, 2 ; 2$ & $d \geq 4$ & $F B^{(3)}$ & $\overline{\left(\bar{\psi} \gamma^{A} D^{A} \psi\right)_{[3]}}$ \\
\hline$\frac{1}{2}, \frac{1}{2}, 2 ; 3$ & $d \geq 4$ & $K^{(12)}\left(B^{(3)}\right)^{2}$ & $\bar{\psi} \gamma^{A B} \partial^{C} \psi \omega^{C A B}$ \\
\hline$\frac{3}{2}, \frac{3}{2}, 0 ; 3$ & $d \geq 4$ & $K^{(12)} B^{(1)} B^{(2)}$ & $\overline{\Psi^{A B} \Psi^{A B} \phi}$ \\
\hline$\frac{3}{2}, \frac{3}{2}, 1 ; 2$ & $d \geq 4$ & $K^{(12)} Z$ & $\left(\bar{\psi}^{A} \psi^{B}-\frac{1}{4} \psi^{C} \gamma^{A B} \psi^{C}\right) F^{A B}$ \\
\hline$\frac{3}{2}, \frac{3}{2}, 1 ; 3$ & $d>4$ & $F B^{(1)} B^{(2)}$ & $\bar{\psi}^{A} \partial^{A} \gamma^{B} \psi^{C} F^{B C}$ \\
\hline$\frac{3}{2}, \frac{3}{2}, 1 ; 4$ & $d \geq 4$ & $K^{(12)} B^{(1)} B^{(2)} B^{(3)}$ & $\bar{\psi}^{A} \partial^{A} \partial^{B} \psi^{C} F^{B C}$ \\
\hline$\frac{3}{2}, \frac{3}{2}, 2 ; 2$ & $d \geq 4$ & $F Z$ & $\left(\bar{\psi}^{A} \gamma^{A B C} D_{\text {Lor }}^{B} \psi^{C}\right)_{[3]}$ \\
\hline$\frac{3}{2}, \frac{3}{2}, 2 ; 3$ & $d>4$ & $K^{(12)} Z B^{(3)}$ & $\bar{\psi}^{C} \partial^{A} \Psi^{B C} \phi^{A B}$ \\
\hline$\frac{3}{2}, \frac{3}{2}, 2 ; 4$ & $d>4$ & $F B^{(1)} B^{(2)} B^{(3)}$ & $\bar{\psi}^{A} \gamma^{B} \Psi^{C E} \partial^{A} \omega^{B C E}$ \\
\hline$\frac{3}{2}, \frac{3}{2}, 2 ; 5$ & $d \geq 4$ & $K^{(12)} B^{(1)} B^{(2)}\left(B^{(3)}\right)^{2}$ & $\bar{\Psi}^{A B} \Psi^{C E} R^{A B C E}$ \\
\hline
\end{tabular}


Non-minimal gravitational interaction of higher-spin fields. Using results in (4.25), (4.26), we can present all interaction vertices of massless higher-spin fermionic field with massless spin-2 field (graviton field). Indeed, the interaction of a massless spin- $\left(s+\frac{1}{2}\right)$ fermionic field with massless spin-2 field is described by the vertex $p_{[3]}^{-}\left(s^{(1)}+\frac{1}{2}, s^{(2)}+\frac{1}{2}, s^{(3)} ; k\right)$ with $s^{(1)}=s^{(2)}=s \geq 2, s^{(3)}=2$. For these values of $s^{(a)}$, we obtain

$$
\mathbf{s}=2 s+2, \quad \mathbf{s}-2 \min \left(s^{(1)}, s^{(2)}, s^{(3)}-1\right)=2 s, \quad s_{\min }=2 .
$$

Using (4.52) in (4.25), (4.26), we get the following allowed values of $k$ :

$$
\begin{array}{ll}
k=2 s-1,2 s+1,2 s+3, & \text { K-vertices; } \\
k=2 s, 2 s+2, & \text { F-vertices. }
\end{array}
$$

We recall that minimal gravitational interaction of low-spin massless fermionic fields is described by F-vertices having $k=2$ (see (4.35),(4.43)). However we note, using (4.53), (4.54), that, for $s \geq 2$, the minimal gravitational interaction, i.e. the case $k=2$, is not allowed. Thus, we see that it is the relations (4.53), (4.54) that leave no place for the minimal gravitational interaction of massless higher-spin fermionic fields. On the other hand, we see, from relations (4.53), (4.54), that there are three type K- and two type F-vertices describing the non-minimal (higher-derivative) gravitational interactions of massless higher-spin fermionic fields and the graviton field.

Table II. Cubic vertices for massless fermionic spin- $\frac{5}{2}$ field and massless bosonic spin $0,1,2$ fields. In the 4 th column, $\phi, \phi^{A}$ stand for the respective spin 0,1 massless fields. $F^{A B}$ and $R^{A B C E}$ stand for the respective Yang-Mills field strength and the Riemann tensor, while $\Psi^{A B C E}$ stands for field strength of spin- $\frac{5}{2}$ field. $\omega_{A}{ }^{B C}$ and $\Omega_{A}{ }^{B C}$ stand for the respective linearized Lorentz connection of spin-2 and spin- $\frac{5}{2}$ fields, $\omega_{A}{ }^{B C}=-\omega_{A} B C, \Omega_{A}{ }^{B C}=-\Omega_{A}{ }^{B C}$. Most of the covariant vertices in the Table are invariant only under linearized on-shell gauge transformations.

\begin{tabular}{|c|c|c|c|}
\hline $\begin{array}{l}\text { Spin values } \\
\text { and number } \\
\text { of derivatives } \\
\frac{5}{2}, \frac{5}{2}, s^{(3)} ; k\end{array}$ & $\begin{array}{c}\text { dimension } \\
\text { of } \\
\text { space-time }\end{array}$ & $\begin{array}{c}\text { Light-cone } \\
\text { vertex } \\
p_{[3]}^{-}\left(\frac{5}{2}, \frac{5}{2}, s^{(3)} ; k\right)\end{array}$ & $\begin{array}{c}\text { Manifestly Lorentz } \\
\text { invariant vertex }\end{array}$ \\
\hline$\frac{5}{2}, \frac{5}{2}, 0 ; 5$ & $d \geq 4$ & $K^{(12)}\left(B^{(1)} B^{(2)}\right)^{2}$ & $\bar{\Psi}^{A B C E} \Psi^{A B C E} \phi$ \\
\hline$\frac{5}{2}, \frac{5}{2}, 1 ; 4$ & $d \geq 4$ & $K^{(12)} Z B^{(1)} B^{(2)}$ & $F^{A B}\left(\bar{\Omega}^{A, C E} \Omega^{B}{ }_{C E}-\bar{\Omega}^{C, E A} \Omega_{C, E}{ }^{B}\right)$ \\
\hline$\frac{5}{2}, \frac{5}{2}, 1 ; 5$ & $d>4$ & $F\left(B^{(1)}\right)^{2}\left(B^{(2)}\right)^{2}$ & $\bar{\Psi}^{A B C D} \gamma^{E} \Psi^{A B C D} \phi^{E}$ \\
\hline$\frac{5}{2}, \frac{5}{2}, 1 ; 6$ & $d \geq 4$ & $K^{(12)}\left(B^{(1)}\right)^{2}\left(B^{(2)}\right)^{2} B^{(3)}$ & $\bar{\Psi}^{A C D E} \Psi^{B C D E} F^{A B}$ \\
\hline$\frac{5}{2}, \frac{5}{2}, 2 ; 3$ & $d \geq 4$ & $K^{(12)} Z^{2}$ & $\operatorname{See}^{A e f} .[35]$ \\
\hline$\frac{5}{2}, \frac{5}{2}, 2 ; 4$ & $d>4$ & $F Z B^{(1)} B^{(2)}$ & \\
\hline$\frac{5}{2}, \frac{5}{2}, 2 ; 5$ & $d>4$ & $K^{(12)} Z B^{(1)} B^{(2)} B^{(3)}$ & $\bar{\Psi}^{A C D E} \gamma^{F} \Psi^{B C D E} \omega^{F A B}$ \\
\hline$\frac{5}{2}, \frac{5}{2}, 2 ; 6$ & $d>4$ & $F\left(B^{(1)}\right)^{2}\left(B^{(2)}\right)^{2} B^{(3)}$ & $\bar{\Psi}_{C D}^{A B} \Psi_{E F}^{C D} R_{A B}^{E F}$ \\
\hline$\frac{5}{2}, \frac{5}{2}, 2 ; 7$ & $d \geq 4$ & $K^{(12)}\left(B^{(1)} B^{(2)} B^{(3)}\right)^{2}$ & \\
\hline
\end{tabular}


We note that $\mathrm{K}$-vertices with $k=\mathbf{s}+1$ correspond to gauge theory cubic interaction vertices built entirely in terms of gauge field strengths. The vertices with $k<\mathbf{s}+1$ cannot be built entirely in terms of gauge field strengths. It is the vertices with $k<\mathbf{s}+1$ that are difficult to construct in Lorentz covariant approaches. The light-cone approach treats all vertices on an equal footing.

\section{Cubic vertices for two massless fermionic fields and one massive bosonic field}

We now consider cubic interaction vertex for three mixed-symmetry fields with the mass values

$$
\left.\mathrm{m}_{1}\right|_{F}=\left.\mathrm{m}_{2}\right|_{F}=0,\left.\quad \mathrm{~m}_{3}\right|_{B} \neq 0
$$

i.e. the massless fermionic fields carry external line indices $a=1,2$, while the massive bosonic field corresponds to $a=3$. Equations for the vertex involving two massless fields can be obtained from Eqs.(3.31) in the limit as $\mathrm{m}_{1} \rightarrow 0, \mathrm{~m}_{2} \rightarrow 0$. The solution for $\mathrm{K}$ - and F-vertices is found to be

$$
\begin{aligned}
& p_{[3]}^{-}=K^{(12)} V^{\mathrm{K}}\left(B_{m}^{(3)} ; Q_{m n}^{(a a+1)}\right), \quad \quad \text { K-vertex }, \\
& p_{[3]}^{-}=F_{n} V^{\mathrm{F}}\left(B_{m}^{(3)} ; Q_{m n}^{(a a+1)}\right), \quad \text { F-vertex },
\end{aligned}
$$

where generating functions $V^{\mathrm{K}}, V^{\mathrm{F}}$ are arbitrary polynomials of forms $B_{m}^{(3)}, Q_{m n}^{(a a+1)}$ and we use the notation 7

$$
\begin{aligned}
& K^{(12)} \equiv \frac{1}{\beta_{1} \beta_{2}} p_{\theta_{1}} \mathbb{P}^{I} \gamma^{I} \gamma_{*} \eta_{2} \\
& F_{n} \equiv \frac{1}{\beta_{1} \beta_{2}} p_{\theta_{1}}\left(\left(\frac{\check{\beta}_{3}}{\beta_{3}} \mathbb{P}^{I}-\gamma^{I J} \mathbb{P}^{J}\right) \alpha_{n}^{(3) I}+\frac{2 \beta_{1} \beta_{2}}{\beta_{3}} \mathrm{~m}_{3} \zeta_{n}^{(3)}\right) \eta_{2} \\
& B_{n}^{(a)} \equiv \frac{\alpha_{n}^{(a) I} \mathbb{P}^{I}}{\beta_{a}}, \quad a=1,2 \\
& B_{n}^{(3)} \equiv \frac{\alpha_{n}^{(3) I} \mathbb{P}^{I}}{\beta_{3}}-\frac{\check{\beta}_{3}}{2 \beta_{3}} \mathrm{~m}_{3} \zeta_{n}^{(3)} \\
& Q_{m n}^{(12)} \equiv \alpha_{m n}^{(12)}-\frac{2}{\mathrm{~m}_{3}^{2}} B_{m}^{(1)} B_{n}^{(2)} \\
& Q_{m n}^{(23)} \equiv \alpha_{m n}^{(23)}-\frac{\zeta_{n}^{(3)}}{\mathrm{m}_{3}} B_{m}^{(2)}+\frac{2}{\mathrm{~m}_{3}^{2}} B_{m}^{(2)} B_{n}^{(3)} \\
& Q_{m n}^{(31)} \equiv \alpha_{m n}^{(31)}+\frac{\zeta_{m}^{(3)}}{\mathrm{m}_{3}} B_{n}^{(1)}+\frac{2}{\mathrm{~m}_{3}^{2}} B_{m}^{(3)} B_{n}^{(1)}
\end{aligned}
$$

The quadratic forms $\alpha_{m n}^{(a b)}$ are defined in (4.8).

\footnotetext{
${ }^{6}$ Our result for the vertex $p_{[3]}^{-}\left(\frac{5}{2}, \frac{5}{2}, 2 ; 7\right)$ (see last row in Table II) implies that there is only one Lorentz covariant vertex $\bar{\Psi}_{C D}^{A B} \Psi_{E F}^{C D} R_{A B}^{E F}$ that gives a non-trivial contribution to the 3-point scattering amplitude. Similar statement for the case of vertex $R_{C D}^{A B} R_{E F}^{C D} R_{A B}^{E F}$ was proved in Ref.[36].

${ }^{7}$ We recall that the short notation like $p_{[3]}^{-}\left(Q^{(a a+1)}\right)$ is used to indicate a dependence of $p_{[3]}^{-}$on $Q^{(12)}, Q^{(23)}, Q^{(31)}$.
} 
From (5.2), (5.3), we see that the prefactor $K^{(12)}$ is homogeneous polynomial in $\mathbb{P}^{I}$, while the prefactors $F_{n}$, the linear forms $B_{n}^{(3)}$ and the quadratic forms $Q_{m n}^{(a a+1)}$ are non-homogeneous polynomials in $\mathbb{P}^{I}$. This implies that, in general, the cubic vertices we obtained are non-homogeneous polynomials in $\mathbb{P}^{I}$. The appearance of massive field interaction vertices involving different powers of derivatives is a well-known fact (see e.g. Refs.[37]). We see that the light-cone approach gives a simple explanation to this phenomenon by means of the prefactors $F_{n}$, the linear forms $B_{n}^{(3)}$, and the quadratic forms $Q_{m n}^{(a a+1)}$. We note that solution for the generating functions $V^{\mathrm{K}}, V^{\mathrm{F}}$ given in (5.2), (5.3) is complete solution, while the solution for the prefactors $K^{(12)}, F_{n}$ given in (5.4), (5.5) is not complete solution. Namely, for the vertices of mixed-symmetry fields there are extra solutions for the prefactors that involve contributions of higher than second order in $\gamma$-matrices. We note also for the vertices of totally symmetric fields those extra solutions are trivial. To understand the remaining characteristic properties of solution (5.2), (5.3), we restrict our attention to cubic vertices for totally symmetric fields.

\subsection{Cubic vertices for totally symmetric two massless fermionic fields and one massive bosonic field}

We now restrict ourselves to cubic vertices for two massless totally symmetric fermionic fields and one massive totally symmetric bosonic field with mass parameters as in (5.1). To consider the totally symmetric fields it is sufficient to use one sort of oscillators. Therefore we set $\nu=1$ in (5.2), (5.3). To simplify the formulas we drop the oscillator's subscript $n=1$ and use the simplified notation for oscillators: $\alpha^{I} \equiv \alpha_{1}^{I}, \zeta \equiv \zeta_{1}$. The cubic vertex for totally symmetric fields under consideration can then be obtained from solution in (5.2), (5.3) by making the identifications

$$
\alpha^{(a) I} \equiv \alpha_{1}^{(a) I}, \quad a=1,2 ; \quad \alpha^{(3) I} \equiv \alpha_{1}^{(3) I} \quad \zeta^{(3)} \equiv \zeta_{1}^{(3)}
$$

and ignoring the contribution of oscillators carrying a subscript $n>1$. Adopting simplified notation (5.11) for the prefactors and forms in (5.6)-(5.10):

$$
F \equiv F_{1}, \quad B^{(a)} \equiv B_{1}^{(a)}, \quad Q^{(a b)} \equiv Q_{11}^{(a b)}, \quad \alpha^{(a b)} \equiv \alpha_{11}^{(a b)},
$$

we see that vertices (5.2), (5.3) take the form

$$
\begin{aligned}
& p_{[3]}^{-}=K^{(12)} V^{\mathrm{K}}\left(B^{(3)} ; Q^{(a a+1)}\right), \quad \text { K-vertex }, \\
& p_{[3]}^{-}=F V^{\mathrm{F}}\left(B^{(3)} ; Q^{(a a+1)}\right), \quad \quad \text { F-vertex },
\end{aligned}
$$

where we use the notation

$$
\begin{aligned}
& F \equiv \frac{1}{\beta_{1} \beta_{2}} p_{\theta_{1}}\left(\left(\frac{\check{\beta}_{3}}{\beta_{3}} \mathbb{P}^{I}-\gamma^{I J} \mathbb{P}^{J}\right) \alpha^{(3) I}+\frac{2 \beta_{1} \beta_{2}}{\beta_{3}} \mathrm{~m}_{3} \zeta^{(3)}\right) \eta_{2} \\
& B^{(a)} \equiv \frac{\alpha^{(a) I} \mathbb{P}^{I}}{\beta_{a}}, \quad a=1,2 \\
& B^{(3)} \equiv \frac{\alpha^{(3) I} \mathbb{P}^{I}}{\beta_{3}}-\frac{\check{\beta}_{3}}{2 \beta_{3}} \mathrm{~m}_{3} \zeta^{(3)} \\
& Q^{(12)} \equiv \alpha^{(12)}-\frac{2}{\mathrm{~m}_{3}^{2}} B^{(1)} B^{(2)}
\end{aligned}
$$




$$
\begin{aligned}
Q^{(23)} & \equiv \alpha^{(23)}-\frac{\zeta^{(3)}}{\mathrm{m}_{3}} B^{(2)}+\frac{2}{\mathrm{~m}_{3}^{2}} B^{(2)} B^{(3)}, \\
Q^{(31)} & \equiv \alpha^{(31)}+\frac{\zeta^{(3)}}{\mathrm{m}_{3}} B^{(1)}+\frac{2}{\mathrm{~m}_{3}^{2}} B^{(3)} B^{(1)},
\end{aligned}
$$

while the quadratic forms $\alpha^{(a b)}$ and prefactor $K^{(12)}$ are defined in (4.17) and (5.4) respectively.

Vertices (5.13), (5.14) describe an interaction of the towers of totally symmetric massive bosonic fields in (2.16) and massless fermionic fields in (2.30). As we have already noted solution for vertices given in (5.13), (5.14) is the complete solution. To understand better the characteristic properties of vertices for totally symmetric fields (5.13),(5.14), we consider vertices for fields with fixed but arbitrary spin values.

\subsubsection{Cubic vertices for totally symmetric fields with fixed but arbitrary spin values}

We now turn to cubic vertices for totally symmetric fields with fixed spin values. This is to say that we consider vertices involving two massless spin $s^{(1)}+\frac{1}{2}$ and $s^{(2)}+\frac{1}{2}$ fermionic fields and one massive spin- $s^{(3)}$ bosonic field having mass parameter $\mathrm{m}_{3}$ :

$$
\begin{array}{lll}
\mathrm{m}_{1}=0, & \mathrm{~m}_{2}=0, & \mathrm{~m}_{3} \neq 0, \\
s^{(1)}+\frac{1}{2}, & s^{(2)}+\frac{1}{2}, & s^{(3)} .
\end{array}
$$

The massless spin- $\left(s^{(1)}+\frac{1}{2}\right)$ and $-\left(s^{(2)}+\frac{1}{2}\right)$ fermionic fields are described by the respective ketvectors $\left|\psi_{s^{(1)}}\right\rangle$ and $\left|\psi_{s^{(2)}}\right\rangle$, while the massive spin- $s^{(3)}$ bosonic field is described by the ket-vector $\left|\phi_{s^{(3)}}\right\rangle$. The ket-vectors of massless fields $\left|\psi_{s^{(a)}}\right\rangle, a=1,2$, can be obtained from (2.23) by the replacement $s \rightarrow s^{(a)}, \alpha^{I} \rightarrow \alpha^{(a) I}, a=1,2$, in (2.23), while the ket-vector of the massive field $\left|\phi_{s^{(3)}}\right\rangle$ can be obtained from (2.11) by the replacement $s \rightarrow s^{(3)}, \alpha^{I} \rightarrow \alpha^{(3) I}, \zeta \rightarrow \zeta^{(3)}$ in (2.11). Taking into account that the ket-vectors $\left|\psi_{s}(a)\right\rangle, a=1,2$, are the respective degree- $s^{(a)}$ homogeneous polynomials in the oscillators $\alpha^{(a) I}$, while the ket-vector $\left|\phi_{s}(3)\right\rangle$ is a degree- $s^{(3)}$ homogeneous polynomial in the oscillators $\alpha^{(3) I}, \zeta^{(3)}$, it is easy to understand that the vertices we are interested in must satisfy the equations

$$
\begin{aligned}
& \left(\alpha^{(a) I} \bar{\alpha}^{(a) I}-s^{(a)}\right)\left|p_{[3]}^{-}\right\rangle=0, \quad a=1,2, \\
& \left(\alpha^{(3) I} \bar{\alpha}^{(3) I}+\zeta^{(3)} \bar{\zeta}^{(3)}-s^{(3)}\right)\left|p_{[3]}^{-}\right\rangle=0 .
\end{aligned}
$$

These equations tell us that the vertices must be degree- $s^{(a)}$ homogeneous polynomials in the respective oscillators. Taking into account that the prefactor $F$ and the linear form $B^{(3)}$ are degree-1 homogeneous polynomials in the oscillators, while the quadratic forms $Q^{(a a+1)}$ are degree-2 homogeneous polynomials in the oscillators, we find the general solution of Eqs.(5.22), (5.23) as:

$$
\begin{array}{ll}
p_{[3]}^{-}\left(s^{(1)}+\frac{1}{2}, s^{(2)}+\frac{1}{2}, s^{(3)} ; w\right)=K^{(12)}\left(B^{(3)}\right)^{w} \prod_{a=1}^{3}\left(Q^{(a a+1)}\right)^{y^{(a+2)}}, & \text { K-vertex }, \\
p_{[3]}^{-}\left(s^{(1)}+\frac{1}{2}, s^{(2)}+\frac{1}{2}, s^{(3)} ; w\right)=F\left(B^{(3)}\right)^{w} \prod_{a=1}^{3}\left(Q^{(a a+1)}\right)^{y^{(a+2)}}, & \text { F-vertex }
\end{array}
$$

where integers $y^{(a)}$ are expressible in terms of the $s^{(a)}$ and integer $w$ by the relations

$$
y^{(a)}=\frac{\mathbf{s}-w}{2}-s^{(a)}, \quad a=1,2,
$$




$$
\begin{array}{ll}
y^{(3)}=\frac{\mathbf{s}+w}{2}-s^{(3)}, & \text { for K-vertex; } \\
y^{(a)}=\frac{\mathbf{s}-w-1}{2}-s^{(a)}, \quad a=1,2, & \\
y^{(3)}=\frac{\mathbf{s}+w+1}{2}-s^{(3)}, & \text { for F-vertex; }
\end{array}
$$

The integer $w$ expresses the freedom of the solution and labels all possible cubic interaction vertices that can be constructed for the fields under consideration. For vertices $(5.24),(5.25)$ to be sensible, we impose the restrictions

$$
\begin{array}{lll}
w \geq 0, & y^{(a)} \geq 0, \quad a=1,2,3 ; & \\
\mathbf{s}-w & \text { even integer, } & \text { for K-vertex; } \\
w \geq 0, & y^{(a)} \geq 0, \quad a=1,2,3 ; & \\
\mathbf{s}-w & \text { odd integer }, & \text { for F-vertex; }
\end{array}
$$

which amount to the requirement that the powers of all forms in (5.24), (5.25) be non-negative integers. We note that using relations (5.26), (5.27) allows rewriting the restrictions (5.28),(5.29) as 8

$$
\begin{array}{ll}
\max \left(0, s^{(3)}-s^{(1)}-s^{(2)}\right) \leq w \leq s^{(3)}-\left|s^{(1)}-s^{(2)}\right|, & \text { for K-vertex; } \\
\max \left(0, s^{(3)}-s^{(1)}-s^{(2)}-1\right) \leq w \leq s^{(3)}-1-\left|s^{(1)}-s^{(2)}\right|, & \text { for F-vertex. }
\end{array}
$$

As compared to vertices for three massless fields (4.20), (4.21), the vertices given in (5.24), (5.25) are non-homogeneous polynomials in $\mathbb{P}^{I}$. An interesting property of vertices $(5.24),(5.25)$ is that the maximal and minimal numbers of powers of the momentum $\mathbb{P}^{I}$, denoted by $k_{\max }$ and $k_{\min }$ respectively, are independent of $w$ and given by

$$
\begin{aligned}
& k_{\max }=\mathbf{s}+1, \quad k_{\min }=1, \quad \text { for K-vertex; } \\
& k_{\max }=\mathbf{s}, \quad k_{\min }=0, \quad \text { for F-vertex. }
\end{aligned}
$$

\section{Cubic vertices for one massless fermionic field, one massless bosonic field and one massive fermionic field}

We proceed with the cubic interaction vertex for three mixed-symmetry fields with the mass values

$$
\left.\mathrm{m}_{1}\right|_{B}=\left.\mathrm{m}_{2}\right|_{F}=0,\left.\quad \mathrm{~m}_{3}\right|_{F} \neq 0,
$$

i.e. the massless bosonic field carries external line index $a=1$, the massless fermionic field carries external line index $a=2$, and the massive fermionic field corresponds to $a=3$. Equations for

\footnotetext{
${ }^{8}$ If $w=0$, then restrictions $(5.30)$ become the restrictions well known in the angular momentum theory: $\mid s^{(1)}-$ $s^{(2)} \mid \leq s^{(3)} \leq s^{(1)}+s^{(2)}$, while restriction (5.31) takes the form $\left|s^{(1)}-s^{(2)}\right| \leq s^{(3)}-1 \leq s^{(1)}+s^{(2)}$.

${ }^{9}$ Expressions for $K^{(12)}, F, B^{(3)}$, and $Q^{(a a+1)}(5.12)$ imply that $k_{\max }=1+w+2 \sum_{a=1}^{3} y^{(a)}$. Taking expressions for $y^{(a)}$ (5.26), (5.27) into account, we find $k_{\max }$ in (5.32), (5.33).
} 
the vertex involving two massless fields can be obtained from Eqs.(3.31) in the limit as $\mathrm{m}_{1} \rightarrow 0$, $\mathrm{m}_{2} \rightarrow 0$. The solution for $\mathrm{K}$ - and $\mathrm{F}$-vertices we found is given by

$$
\begin{array}{ll}
p_{[3]}^{-}=K^{(23)} V^{\mathrm{K}}\left(B_{n}^{(3)} ; Q_{m n}^{(a a+1)}\right), & \text { K vertex }, \\
p_{[3]}^{-}=F_{n} V^{\mathrm{F}}\left(B_{n}^{(3)} ; Q_{m n}^{(a a+1)}\right), & \text { F vertex },
\end{array}
$$

where generating functions $V^{\mathrm{K}}, V^{\mathrm{F}}$ are arbitrary polynomials of forms $B_{n}^{(3)}, Q_{m n}^{(a a+1)}$ and we use the notation

$$
\begin{gathered}
K^{(23)} \equiv \frac{1}{\beta_{2} \beta_{3}} p_{\theta_{2}}\left(\mathbb{P}^{I} \gamma^{I} \gamma_{*}+\beta_{2} \mathrm{~m}_{3}\right) \eta_{3} \\
F_{n} \equiv \frac{1}{\beta_{2} \beta_{3}} p_{\theta_{2}}\left(\frac{\check{\beta}_{1}}{\beta_{1}} \mathbb{P}^{I}-\gamma^{I J} \mathbb{P}^{J}+\beta_{2} \mathrm{~m}_{3} \gamma^{I} \gamma_{*}\right) \eta_{3} \alpha_{n}^{(1) I}-\frac{2}{\mathrm{~m}_{3}} K^{(23)} B_{n}^{(1)} \\
B_{n}^{(a)} \equiv \frac{\alpha_{n}^{(a) I} \mathbb{P}^{I}}{\beta_{a}}, \quad a=1,2 \\
B_{n}^{(3)} \equiv \frac{\alpha_{n}^{(3) I} \mathbb{P}^{I}}{\beta_{3}}-\frac{\check{\beta}_{3}}{2 \beta_{3}} \mathrm{~m}_{3} \zeta_{n}^{(3)} \\
Q_{m n}^{(12)} \equiv \alpha_{m n}^{(12)}-\frac{2}{\mathrm{~m}_{3}^{2}} B_{m}^{(1)} B_{n}^{(2)}, \\
Q_{m n}^{(23)} \equiv \alpha^{(23)}-\frac{\zeta_{n}^{(3)}}{\mathrm{m}_{3}} B_{m}^{(2)}+\frac{2}{\mathrm{~m}_{3}^{2}} B_{m}^{(2)} B_{n}^{(3)} \\
Q_{m n}^{(31)} \equiv \alpha_{m n}^{(31)}+\frac{\zeta_{m}^{(3)}}{\mathrm{m}_{3}} B_{n}^{(1)}+\frac{2}{\mathrm{~m}_{3}^{2}} B_{m}^{(3)} B_{n}^{(1)}
\end{gathered}
$$

The quadratic forms $\alpha_{m n}^{(a b)}$ are defined in (4.8).

We note that the prefactors $K^{(23)}$ and $F_{n}$, the linear forms $B_{n}^{(3)}$, and quadratic forms $Q_{m n}^{(a a+1)}$ are non-homogeneous polynomials in the momentum $\mathbb{P}^{I}$. This implies that the cubic interaction vertices given in (6.2), (6.3) are also non-homogeneous polynomials in the momentum $\mathbb{P}^{I}$. We note that solution for $V^{\mathrm{K}}, V^{\mathrm{F}}$ given in (6.2), (6.3) is complete solution, while the solution for the prefactors $K^{(23)}, F_{n}$ given in (6.4), (6.5) is not complete solution. This is to say that for the vertices of mixed-symmetry fields there are extra solutions for the prefactors that involve contributions of higher than second order in $\gamma$-matrices.

\subsection{Cubic vertices for totally symmetric one massless fermionic field, one massless bosonic field and one massive fermionic field}

We proceed with the study of vertices for three totally symmetric fields with the mass values given in (6.1), i.e. the massless bosonic field carries external line index $a=1$, the massless fermionic field carries external line index $a=2$, and the massive fermionic field corresponds to $a=3$. To consider the totally symmetric fields it is sufficient to use one sort of oscillators. Therefore we set $\nu=1$ in 6.2), (6.3). To simplify the formulas we drop the oscillator's subscript $n=1$ and use the simplified notation for oscillators: $\alpha^{(a) I} \equiv \alpha_{1}^{(a) I}, \zeta^{(a)} \equiv \zeta_{1}^{(a)}$. The cubic interaction vertices for 
totally symmetric fields under consideration can then be obtained from solution $(6.2),(6.3)$. The vertices take the form

$$
\begin{array}{ll}
p_{[3]}^{-}=K^{(23)} V^{\mathrm{K}}\left(B^{(3)} ; Q^{(a a+1)}\right), & \mathrm{K} \text { vertex }, \\
p_{[3]}^{-}=F V^{\mathrm{F}}\left(B^{(3)} ; Q^{(a a+1)}\right), & \text { F vertex },
\end{array}
$$

where we use the notation

$$
\begin{aligned}
& F \equiv \frac{1}{\beta_{2} \beta_{3}} p_{\theta_{2}}\left(\frac{\check{\beta}_{1}}{\beta_{1}} \mathbb{P}^{I}-\gamma^{I J} \mathbb{P}^{J}+\beta_{2} \mathrm{~m}_{3} \gamma^{I} \gamma_{*}\right) \eta_{3} \alpha^{(1) I}-\frac{2}{\mathrm{~m}_{3}} K^{(23)} B^{(1)} \\
& B^{(a)} \equiv \frac{\alpha^{(a) I} \mathbb{P}^{I}}{\beta_{a}}, \quad a=1,2 \\
& B^{(3)} \equiv \frac{\alpha^{(3) I} \mathbb{P}^{I}}{\beta_{3}}-\frac{\check{\beta}_{3}}{2 \beta_{3}} \mathrm{~m}_{3} \zeta^{(3)} \\
& Q^{(12)} \equiv \alpha^{(12)}-\frac{2}{\mathrm{~m}_{3}^{2}} B^{(1)} B^{(2)} \\
& Q^{(23)} \equiv \alpha^{(23)}-\frac{\zeta^{(3)}}{\mathrm{m}_{3}} B^{(2)}+\frac{2}{\mathrm{~m}_{3}^{2}} B^{(2)} B^{(3)} \\
& Q^{(31)} \equiv \alpha^{(31)}+\frac{\zeta^{(3)}}{\mathrm{m}_{3}} B^{(1)}+\frac{2}{\mathrm{~m}_{3}^{2}} B^{(3)} B^{(1)}
\end{aligned}
$$

while $\alpha^{(a b)}$ and $K^{(23)}$ are defined in (4.17) and (6.4) respectively.

Vertices (6.11), (6.12) describe an interaction of the towers of bosonic and fermionic fields. We note that solution for vertices given in (6.11), (6.12) provide the complete list of parity invariant cubic vertices for totally symmetric fields with the mass parameters shown in (6.1). To understand the remaining characteristic properties of solution (6.11), (6.12), we consider vertices for totally symmetric fields with fixed spin values.

\subsubsection{Vertices for totally symmetric fields with fixed but arbitrary spin values}

In this section, we restrict ourselves to cubic vertices for totally symmetric fields with fixed spin values. This is to say that we consider vertices for one massless spin- $s^{(1)}$ bosonic field, one massless spin- $\left(s^{(2)}+\frac{1}{2}\right)$ fermionic field and one massive spin- $\left(s^{(3)}+\frac{1}{2}\right)$ fermionic field. This, we consider vertices involving fields with the following spin and mass values:

$$
\begin{array}{lll}
\mathrm{m}_{1}=0, & \mathrm{~m}_{2}=0, & \mathrm{~m}_{3} \neq 0, \\
s^{(1)}, & s^{(2)}+\frac{1}{2}, & s^{(3)}+\frac{1}{2} .
\end{array}
$$

The massless spin- $s^{(1)}$ bosonic field and massless spin- $\left(s^{(2)}+\frac{1}{2}\right)$ fermionic field are described by the respective ket-vectors $\left|\phi_{s^{(1)}}\right\rangle$ and $\left|\psi_{s^{(2)}}\right\rangle$, while the massive spin- $\left(s^{(3)}+\frac{1}{2}\right)$ fermionic field is described by the ket-vector $\left|\psi_{s^{(3)}}\right\rangle$. The ket-vectors of massless fields $\left|\phi_{s^{(1)}}\right\rangle,\left|\psi_{s^{(2)}}\right\rangle$ can be obtained from the respective expressions in (2.10), (2.23) by the replacement $s \rightarrow s^{(a)}, \alpha^{I} \rightarrow \alpha^{(a) I}, a=1,2$, 
while the ket-vector of the massive field $\left|\psi_{s^{(3)}}\right\rangle$ can be obtained from (2.23) by the replacement $s \rightarrow s^{(3)}, \alpha^{I} \rightarrow \alpha^{(3) I}, \zeta \rightarrow \zeta^{(3)}$. Taking into account that the ket-vectors $\left|\phi_{s}(1)\right\rangle$ and $\left|\psi_{s}(2)\right\rangle$ are the respective degree- $s^{(1)}$ and degree- $s^{(2)}$ homogeneous polynomials in the oscillators $\alpha^{(a) I}, a=1,2$, while the ket-vector $\left|\psi_{s^{(3)}}\right\rangle$ is a degree- $s^{(3)}$ homogeneous polynomial in the oscillators $\alpha^{(3) I}, \zeta^{(3)}$, it is easy to understand that the vertices we are interested in must satisfy the equations

$$
\begin{aligned}
& \left(\alpha^{(a) I} \bar{\alpha}^{(a) I}-s^{(a)}\right)\left|p_{[3]}^{-}\right\rangle=0, \quad a=1,2, \\
& \left(\alpha^{(3) I} \bar{\alpha}^{(3) I}+\zeta^{(3)} \bar{\zeta}^{(3)}-s^{(3)}\right)\left|p_{[3]}^{-}\right\rangle=0 .
\end{aligned}
$$

These equations tell us that the vertices must be a degree- $s^{(a)}$ homogeneous polynomials in the respective oscillators. Taking into account that the prefactor $F$ and the form $B^{(3)}$ are degree-1 homogeneous polynomials in the oscillators, while the forms $Q^{(a a+1)}$ are degree-2 homogeneous polynomials in the oscillators, we find the general solution of Eqs. (6.20), (6.21) as:

$$
\begin{array}{ll}
p_{[3]}^{-}\left(s^{(1)}, s^{(2)}+\frac{1}{2}, s^{(3)}+\frac{1}{2} ; w\right)=K^{(23)}\left(B^{(3)}\right)^{w} \prod_{a=1}^{3}\left(Q^{(a a+1)}\right)^{y^{(a+2)}}, & \text { K vertex }, \\
p_{[3]}^{-}\left(s^{(1)}, s^{(2)}+\frac{1}{2}, s^{(3)}+\frac{1}{2} ; w\right)=F\left(B^{(3)}\right)^{w} \prod_{a=1}^{3}\left(Q^{(a a+1)}\right)^{y^{(a+2)}}, & \text { F vertex }
\end{array}
$$

where integers $y^{(a)}$ are expressible in terms of the $s^{(a)}$ and integer $w$ by the relations

$$
\begin{array}{ll}
y^{(a)}=\frac{\mathbf{s}-w}{2}-s^{(a)}, \quad a=1,2, & \\
y^{(3)}=\frac{\mathbf{s}+w}{2}-s^{(3)}, & \text { for K-vertex; } \\
y^{(1)}=\frac{\mathbf{s}-w+1}{2}-s^{(1)} & \\
y^{(2)}=\frac{\mathbf{s}-w-1}{2}-s^{(2)}, & \text { for F-vertex; } \\
y^{(3)}=\frac{\mathbf{s}+w-1}{2}-s^{(3)}, &
\end{array}
$$

The integer $w$ expresses the freedom of the solution and labels all possible cubic interaction vertices that can be constructed for the fields under consideration. For vertices (6.24), (6.25) to be sensible, we impose the restrictions

$$
\begin{array}{lll}
w \geq 0, & y^{(a)} \geq 0, \quad a=1,2,3 ; & \\
\mathbf{s}-w & \text { even integer }, & \text { for K-vertex; } \\
w \geq 0, & y^{(a)} \geq 0, \quad a=1,2,3 ; & \\
\mathbf{s}-w & \text { odd integer }, & \text { for F-vertex; }
\end{array}
$$

which amount to the requirement that the powers of all forms in (6.22), (6.23) be non-negative integers. We note that using relations (6.24), (6.25) allows rewriting the restrictions (6.26), (6.27) 
as 10

$$
\begin{array}{ll}
\max \left(0, s^{(3)}-s^{(1)}-s^{(2)}\right) \leq w \leq s^{(3)}-\left|s^{(1)}-s^{(2)}\right|, & \text { for K-vertex; } \\
\max \left(0, s^{(3)}-s^{(1)}-s^{(2)}+1\right) \leq w \leq s^{(3)}-\left|s^{(1)}-s^{(2)}-1\right|, & \text { for F-vertex }
\end{array}
$$

The maximal and minimal numbers of powers of the momentum $\mathbb{P}^{I}$, denoted by $k_{\max }$ and $k_{\min }$ respectively, are independent of $w$ and given by

$$
\begin{array}{lll}
k_{\max }=\mathbf{s}+1, & k_{\min }=0, & \text { for K-vertex } \\
k_{\max }=\mathbf{s}, & k_{\min }=0, & \text { for F-vertex. }
\end{array}
$$

\section{Cubic vertices for one massless bosonic field and two massive fermionic fields with the same mass values}

The case under consideration is most interesting because it involves the Yang-Mills and gravitational interactions of massive arbitrary spin fermionic fields as particular cases. We now consider the cubic interaction vertex for mixed-symmetry fields having the following mass values,

$$
\left.\mathrm{m}_{1}\right|_{F}=\left.\mathrm{m}_{2}\right|_{F} \equiv \mathrm{m} \neq 0,\left.\quad \mathrm{~m}_{3}\right|_{B}=0
$$

i.e. the massive fermionic fields having the same values of mass parameter carry external line indices $a=1,2$, while the massless bosonic field corresponds to $a=3$. The solution for $\mathrm{K}$ - and F-cubic vertices we found is given by

$$
\begin{array}{ll}
p_{[3]}^{-}=K^{(12)} V^{\mathrm{K}}\left(L_{n}^{(1)}, L_{n}^{(2)}, B_{n}^{(3)} ; Q_{m n}^{(12)} ; Z_{m n q}\right), & \text { K-vertex; } \\
p_{[3]}^{-}=F_{0 n}^{(3)} V^{\mathrm{F}}\left(L_{n}^{(1)}, L_{n}^{(2)}, B_{n}^{(3)} ; Q_{m n}^{(12)} ; Z_{m n q}\right), & \text { F-vertex; }
\end{array}
$$

where generating functions $V^{\mathrm{K}}, V^{\mathrm{F}}$ are arbitrary polynomials of linear, quadratic, and cubic forms. We use the notation

$$
\begin{aligned}
& K^{(12)}=\frac{1}{\beta_{1} \beta_{2}} p_{\theta_{1}}\left(\mathbb{P}^{I} \gamma^{I} \gamma_{*}+\mathrm{m} \check{\beta}_{3}\right) \eta_{2} \\
& F_{0 n}^{(3)}=\frac{1}{\beta_{1} \beta_{2}} p_{\theta_{1}}\left(\frac{\check{\beta}_{3}}{\beta_{3}} \mathbb{P}^{I}-\gamma^{I J} \mathbb{P}^{J}-\mathrm{m} \beta_{3} \gamma^{I} \gamma_{*}\right) \eta_{2} \alpha_{n}^{(3) I} \\
& L_{n}^{(1)} \equiv B_{n}^{(1)}-\frac{1}{2} \mathrm{~m} \zeta_{n}^{(1)}, \quad \quad L_{n}^{(2)} \equiv B_{n}^{(2)}+\frac{1}{2} \mathrm{~m} \zeta_{n}^{(2)} \\
& B_{n}^{(a)} \equiv \frac{\alpha_{n}^{(a) I} \mathbb{P}^{I}}{\beta_{a}}-\frac{\check{\beta}_{a}}{2 \beta_{a}} \mathrm{~m} \zeta_{n}^{(a)}, \quad a \\
& B_{n}^{(3)} \equiv \frac{\alpha_{n}^{(3) I} \mathbb{P}^{I}}{\beta_{3}},
\end{aligned}
$$

\footnotetext{
${ }^{10}$ If $w=0$, then restrictions $(6.28)$ becomes the restrictions well known in the angular momentum theory: $\mid s^{(1)}-$ $s^{(2)} \mid \leq s^{(3)} \leq s^{(1)}+s^{(2)}$, while restriction 6.29) takes the form $\left|s^{(1)}-s^{(2)}-1\right| \leq s^{(3)} \leq s^{(1)}+s^{(2)}-1$
} 


$$
\begin{aligned}
& Q_{m n}^{(12)} \equiv \alpha_{n}^{(12)}-\frac{\zeta_{n}^{(2)}}{\mathrm{m}} B_{m}^{(1)}+\frac{\zeta_{m}^{(1)}}{\mathrm{m}} B_{n}^{(2)}, \\
& Z_{m n q} \equiv L_{m}^{(1)} \alpha_{n q}^{(23)}+L_{n}^{(2)} \alpha_{q m}^{(31)}+B_{q}^{(3)}\left(\alpha_{m n}^{(12)}-\zeta_{m}^{(1)} \zeta_{n}^{(2)}\right),
\end{aligned}
$$

where the quadratic forms $\alpha_{m n}^{(a b)}$ are defined in (4.8).

From (7.4)-(7.10), we see that only the linear forms $B_{n}^{(3)}(7.8)$ are homogeneous polynomials in the momentum $\mathbb{P}^{I}$, while the prefactors $K^{(12)}, F_{0 n}^{(3)}$, the linear forms $L_{n}^{(1)}, L_{n}^{(2)}$, the quadratic forms $Q_{m n}^{(12)}$, and the cubic forms $Z_{m n q}$ are non-homogeneous polynomials in $\mathbb{P}^{I}$. Because the prefactors $K^{(12)}, F_{0 n}^{(3)}$ are non-homogeneous polynomials in $\mathbb{P}^{I}$ all cubic interaction vertices for fields with mass parameters as in (7.1) are also non-homogeneous polynomials in $\mathbb{P}^{I}$. Note also that, because the linear forms $B_{n}^{(3)}$ are homogeneous polynomials in $\mathbb{P}^{I}$, the minimal number of powers of $\mathbb{P}^{I}$ in cubic vertices $(7.2),(7.3)$ is not equal to zero in general. As before, solution for the generating functions $V^{\mathrm{K}}, V^{\mathrm{F}}$ given in (7.2), (7.3) is complete solution, while the solution for the prefactors $K^{(12)}, F_{0 n}^{(3)}$ given in (7.4),(7.5) is not complete solution. Namely, for the vertices of mixed-symmetry fields there are extra solutions for the prefactors that involve contributions of higher than second order in $\gamma$-matrices.

To discuss the remaining important properties of the solutions given (7.2), (7.3) we restrict our attention to cubic vertices for the totally symmetric fields.

\subsection{Cubic vertices for totally symmetric one massless bosonic field and two massive fermionic fields with the same mass values}

We proceed with the study of vertices for three totally symmetric fields with the mass values given in (7.1), i.e. the massive fermionic fields having the same values of mass parameter carry external line indices $a=1,2$, while the massless bosonic field carries external line index $a=3$. As before, to consider the totally symmetric fields, it is sufficient to use one sort of oscillators. Therefore we set $\nu=1$ in (7.2), (7.3) and ignore the contribution of the oscillators with $n>1$. Also, to simplify the formulas we drop the oscillator's subscript $n=1$ and use the simplified notation for oscillators: $\alpha^{(a) I} \equiv \alpha_{1}^{(a) I}, \zeta^{(a)} \equiv \zeta_{1}^{(a)}$. In doing so, we obtain, using the solutions given in (7.2), (7.3), the cubic interaction vertices for the totally symmetric fields,

$$
\begin{aligned}
& p_{[3]}^{-}=K^{(12)} V^{\mathrm{K}}\left(L^{(1)}, L^{(2)}, B^{(3)} ; Q^{(12)} ; Z\right), \quad \quad \text { K-vertex; } \\
& p_{[3]}^{-}=F_{0}^{(3)} V^{\mathrm{F}}\left(L^{(1)}, L^{(2)}, B^{(3)} ; Q^{(12)} ; Z\right), \quad \text { F-vertex; }
\end{aligned}
$$

where we use the notation

$$
\begin{aligned}
F_{0}^{(3)} & =\frac{1}{\beta_{1} \beta_{2}} p_{\theta_{1}}\left(\frac{\check{\beta}_{3}}{\beta_{3}} \mathbb{P}^{I}-\gamma^{I J} \mathbb{P}^{J}-\mathrm{m} \beta_{3} \gamma^{I} \gamma_{*}\right) \eta_{2} \alpha^{(3) I}, \\
L^{(1)} & \equiv B^{(1)}-\frac{1}{2} \mathrm{~m} \zeta^{(1)}, \quad L^{(2)} \equiv B^{(2)}+\frac{1}{2} \mathrm{~m} \zeta^{(2)}, \\
B^{(a)} & \equiv \frac{\alpha^{(a) I} \mathbb{P}^{I}}{\beta_{a}}-\frac{\check{\beta}_{a}}{2 \beta_{a}} \mathrm{~m} \zeta^{(a)}, \quad a=1,2 ; \\
B^{(3)} & \equiv \frac{\alpha^{(3) I} \mathbb{P}^{I}}{\beta_{3}},
\end{aligned}
$$




$$
\begin{aligned}
& Q^{(12)} \equiv \alpha^{(12)}-\frac{\zeta^{(2)}}{\mathrm{m}} B^{(1)}+\frac{\zeta^{(1)}}{\mathrm{m}} B^{(2)}, \\
& Z \equiv L^{(1)} \alpha^{(23)}+L^{(2)} \alpha^{(31)}+B^{(3)}\left(\alpha^{(12)}-\zeta^{(1)} \zeta^{(2)}\right),
\end{aligned}
$$

while the quadratic forms $\alpha^{(a b)}$ and the prefactor $K^{(12)}$ are defined in (4.17) and (7.4) respectively. Solutions for vertices given in (7.11),(7.12) are the complete solutions, i.e., vertices given in (7.11), (7.12) provide the complete list of parity invariant cubic vertices for the totally symmetric fields with the mass parameters given in (7.1).

To discuss the remaining important properties of the solutions given in (7.11), (7.12) we restrict our attention to cubic vertices for totally symmetric fields with fixed spin values.

\subsubsection{Cubic vertices for totally symmetric fields with fixed but arbitrary spin values}

In this section, we restrict ourselves to cubic interaction vertices for the totally symmetric fields with fixed but arbitrary spin values and with mass values given in (7.1). This is to say that we consider vertices involving two massive spin $s^{(1)}+\frac{1}{2}$ and $s^{(2)}+\frac{1}{2}$ fermionic fields having the same mass parameter $m$ and one massless spin- $s^{(3)}$ bosonic field,

$$
\begin{array}{lll}
\mathrm{m}_{1}=\mathrm{m}, & \mathrm{m}_{2}=\mathrm{m}, \quad \mathrm{m} \neq 0, & \mathrm{~m}_{3}=0, \\
s^{(1)}+\frac{1}{2}, & s^{(2)}+\frac{1}{2}, & s^{(3)} .
\end{array}
$$

Two massive spin $s^{(1)}+\frac{1}{2}$ and $s^{(2)}+\frac{1}{2}$ fermionic fields are described by the respective ket-vectors $\left|\psi_{s^{(1)}}\right\rangle$ and $\left|\psi_{s^{(2)}}\right\rangle$, while one massless spin- $s^{(3)}$ bosonic field is described by the ket-vector $\left|\phi_{s^{(3)}}\right\rangle$. The ket-vectors of massive fields $\left|\psi_{s^{(a)}}\right\rangle, a=1,2$, can be obtained from (2.23) by the replacement $s \rightarrow s^{(a)}, \alpha^{I} \rightarrow \alpha^{(a) I}, \zeta \rightarrow \zeta^{(a)}, a=1,2$, while the ket-vector of massless field $\left|\phi_{s}(3)\right\rangle$ can be obtained from (2.10) by the replacement $s \rightarrow s^{(3)}, \alpha^{I} \rightarrow \alpha^{(3) I}$. Taking into account that the ketvectors $\left|\psi_{s^{(a)}}\right\rangle, a=1,2$, are the respective degree- $s^{(a)}$ homogeneous polynomials in the oscillators $\alpha^{(a) I}, \zeta^{(a)}$, while the ket-vector $\left|\phi_{s^{(3)}}\right\rangle$ is a degree- $s^{(3)}$ homogeneous polynomial in the oscillator $\alpha^{(3) I}$, it is easy to understand that the vertices we are interested in must satisfy the equations

$$
\begin{aligned}
& \left(\alpha^{(a) I} \bar{\alpha}^{(a) I}+\zeta^{(a)} \bar{\zeta}^{(a)}-s^{(a)}\right)\left|p_{[3]}^{-}\right\rangle=0, \quad a=1,2, \\
& \left(\alpha^{(3) I} \bar{\alpha}^{(3) I}-s^{(3)}\right)\left|p_{[3]}^{-}\right\rangle=0 .
\end{aligned}
$$

These equations tell us that the vertices must be a degree- $s^{(a)}$ homogeneous polynomials in the respective oscillators. Taking into account that the prefactor $F_{0}^{(3)}$ and the forms $L^{(1)}, L^{(2)}, B^{(3)}$ given in (7.13)-(7.16) are degree-1 homogeneous polynomials in the oscillators, while quadratic form $Q^{(12)}(7.17)$ and cubic form $Z$ (7.18) are the respective degree-2 and degree-3 homogeneous polynomials in the oscillators, we find the general solution of Eqs.(7.20), (7.21) as

$$
\begin{aligned}
p_{[3]}^{-}\left(s^{(1)}+\right. & \left.\frac{1}{2}, s^{(2)}+\frac{1}{2}, s^{(3)} ; k_{\min }, k_{\max }\right) \\
& =K^{(12)}\left(L^{(1)}\right)^{w^{(1)}}\left(L^{(2)}\right)^{w^{(2)}}\left(B^{(3)}\right)^{w^{(3)}}\left(Q^{(12)}\right)^{y^{(3)}} Z^{y} \\
& =F_{0}^{(3)}\left(L^{(1)}\right)^{w^{(1)}}\left(L^{(2)}\right)^{w^{(2)}}\left(B^{(3)}\right)^{w^{(3)}}\left(Q^{(12)}\right)^{y^{(3)}} Z^{y}
\end{aligned}
$$

where the parameters $w^{(1)}, w^{(2)}, w^{(3)}, y^{(3)}, y$ are given by

$$
w^{(1)}=k_{\max }-k_{\min }-s^{(2)}-1,
$$




$$
\begin{aligned}
& w^{(2)}=k_{\max }-k_{\min }-s^{(1)}-1, \\
& w^{(3)}=k_{\min } \\
& y^{(3)}=\mathbf{s}-2 s^{(3)}-k_{\max }+2 k_{\min }+1, \\
& y=s^{(3)}-k_{\min }, \\
& w^{(1)}=k_{\max }-k_{\min }-s^{(2)}-1, \\
& w^{(2)}=k_{\max }-k_{\min }-s^{(1)}-1, \\
& w^{(3)}=k_{\min }, \\
& y^{(3)}=\mathbf{s}-2 s^{(3)}-k_{\max }+2 k_{\min }+2, \\
& y=s^{(3)}-k_{\min }-1,
\end{aligned}
$$

and $\mathrm{s}$ is defined in (4.22). Integers $k_{\min }$ and $k_{\max }$ in (7.22)-(7.25) are the freedom in our solution. In general, vertices $(7.22),(7.23)$ are non-homogeneous polynomials in the momentum $\mathbb{P}^{I}$ and the integers $k_{\min }$ and $k_{\max }$ are the respective minimal and maximal numbers of powers of the momentum $\mathbb{P}^{I}$ in $(7.22),(7.23)$. For vertices $(7.22),(7.23)$ to be sensible, we should impose the restrictions

$$
w^{(a)} \geq 0, \quad a=1,2,3 ; \quad y^{(3)} \geq 0, \quad y \geq 0,
$$

which amount to requiring the powers of all forms in $(7.22),(7.23)$ to be non-negative integers. Using (7.24), (7.25), restrictions (7.26) can be rewritten in a more convenient form as

$$
\begin{array}{lr}
k_{\min }+\max _{a=1,2} s^{(a)}+1 \leq k_{\max } \leq \mathbf{s}-2 s^{(3)}+2 k_{\min }+1, \\
0 \leq k_{\min } \leq s^{(3)}, & \text { for K-vertices; } \\
k_{\min }+\max _{a=1,2} s^{(a)}+1 \leq k_{\max } \leq \mathbf{s}-2 s^{(3)}+2 k_{\min }+2, \\
0 \leq k_{\min } \leq s^{(3)}-1, & \text { for F-vertices. }
\end{array}
$$

Cubic vertices in (7.22), (7.23) and restrictions on allowed values of $k_{\max }$ and $k_{\min }$ in (7.27), (7.28) provide the complete list of parity invariant cubic vertices for the totally symmetric fields with spin and mass values given in (7.19).

\subsubsection{Interaction of massive arbitrary spin fermionic field with massless scalar field}

We now use results in Section 7.1.1 to discuss cubic vertices for two fermionic fields and one scalar field. In doing so, we present the list of all parity invariant cubic vertices for the totally symmetric massive arbitrary spin- $\left(s+\frac{1}{2}\right)$ fermionic field interacting with the massless scalar field. This is to say that we consider vertices (7.22), (7.23) with the mass and spin values given by

$$
\begin{array}{lll}
\mathrm{m}_{1}=\mathrm{m}, & \mathrm{m}_{2}=\mathrm{m}, & \mathrm{m}_{3}=0, \\
s^{(1)}+\frac{1}{2}, & s^{(2)}+\frac{1}{2}, & s^{(3)}=0,
\end{array}
$$

where $\mathrm{m} \neq 0, s^{(1)}=s^{(2)}=s$. 
Allowed spin values and powers of derivatives $k_{\min }, k_{\max }$ should satisfy restrictions in (7.27), (7.28). Plugging $s^{(3)}=0$ in the last restriction in (7.28), we learn that F-vertices are not allowed. Plugging $s^{(3)}=0$ in the last restriction in (7.27), we learn that all allowed K-vertices take $k_{\min }=0$. Plugging $k_{\min }=0$ into the 1 st restrictions in (7.27), we summarize the allowed values of $k_{\min }$ and $k_{\max }$ for K-vertices:

$$
k_{\min }=0, \quad s+1 \leq k_{\max } \leq 2 s+1, \quad \text { for K-vertices. }
$$

Using $k_{\min }=0$ in (7.22), (7.24), we get the following cubic interactions K-vertices:

$$
p_{[3]}^{-}\left(s+\frac{1}{2}, s+\frac{1}{2}, 0 ; 0, k_{\max }\right)=K^{(12)}\left(L^{(1)} L^{(2)}\right)^{k_{\max }-s-1}\left(Q^{(12)}\right)^{2 s+1-k_{\max }} .
$$

Vertices (7.31) supplemented with inequalities for allowed values of $k_{\max }$ in (7.30) constitute the complete list of the parity invariant cubic vertices that can be built for two totally symmetric massive spin- $\left(s+\frac{1}{2}\right)$ fermionic fields and one massless scalar field.

For the case of $s=0$, we obtain only one allowed value of $k_{\max }=1$ in (7.30) and the corresponding vertex given by

$$
p_{[3]}^{-}\left(\frac{1}{2}, \frac{1}{2}, 0 ; 0,1\right)=K^{(12)}
$$

describes interaction of spin- $\frac{1}{2}$ fermionic field with scalar field. Appropriate covariant vertex is well known, $\mathcal{L}_{[3]}=\bar{\psi} \psi \phi$.

For the case of $s>0$, we obtain $s+1$ interaction vertices labelled by allowed values of $k_{\max }$ in (7.30). Note that quadratic form $Q^{(12)}(7.17)$ does not have a smooth massless limit $(\mathrm{m} \rightarrow 0)$. This implies that the vertex for the massive spin $s+\frac{1}{2} \geq \frac{3}{2}$ fermionic field (7.31) does not admit a sensible massless limit when $k_{\max }<2 s+1$. In other words, there are $s$ vertices with $s+1 \leq$ $k_{\max }<2 s+1$ which do not have a smooth massless limit and one vertex with $k_{\max }=2 s+1$ which has smooth massless limit. For example, covariant vertex for one scalar field and two spin- $\frac{3}{2}$ fermionic fields which has smooth massless limit takes the form $\mathcal{L}=\bar{\Psi}^{A B} \Psi^{A B} \phi$, where $\Psi^{A B}$ is defined in (4.39).

\subsubsection{Yang-Mills interaction of massive arbitrary spin fermionic field}

We now apply our results in Section 7.1.1 to the discussion of the Yang-Mills interaction of the massive arbitrary spin fermionic fields 11 Since the Yang-Mills interaction of massive fermionic fields are described by F-vertices we restrict our attention to the F-vertices in what follows. We first present the list of all cubic F-vertices for the massive arbitrary spin- $\left(s+\frac{1}{2}\right)$ fermionic field interacting with the massless spin-1 field (Yang-Mills field). This is to say that we consider Fvertices (7.23) with

$$
\begin{array}{lll}
\mathrm{m}_{1}=\mathrm{m}, & \mathrm{m}_{2}=\mathrm{m}, & \mathrm{m}_{3}=0, \\
s^{(1)}+\frac{1}{2}, & s^{(2)}+\frac{1}{2}, & s^{(3)}=1,
\end{array}
$$

where $\mathrm{m} \neq 0, s^{(1)}=s^{(2)}=s$. The 2 nd restrictions in (7.28) lead to one allowed value of $k_{\min }=0$. Plugging such $k_{\min }$ into the 1 st inequalities in (7.28), we summarize the allowed values of $k_{\min }$ and $k_{\max }$ for F-vertices:

$$
k_{\min }=0, \quad s+1 \leq k_{\max } \leq 2 s+1, \quad s \geq 0, \quad \text { for F-vertices }
$$

\footnotetext{
${ }^{11}$ Discussion of gauge invariant formulation of massive arbitrary spin fermionic fields in constant electromagnetic field may be found in [38].
} 
We now discuss those vertices from the list in (7.34) that correspond to the Yang-Mills interaction of the massive arbitrary spin fermionic field. We consider various spin fields in turn.

a) Spin- $\frac{1}{2}$ field $(s=0)$. Plugging $s=0$ into (7.34) we obtain $k_{\max }=1$. Thus we see that the cubic vertex of the Yang-Mills interaction of the massive spin- $\frac{1}{2}$ fermionic field has $k_{\min }=0, k_{\max }=1$. Using such $k_{\min }, k_{\max }$ in (7.23), (7.25), we get the Yang-Mills interaction vertex for the massive spin- $\frac{1}{2}$ fermionic field

$$
p_{[3]}^{-}\left(\frac{1}{2}, \frac{1}{2}, 1 ; 0,1\right)=F_{0}^{(3)} .
$$

b) Spin $s+\frac{1}{2} \geq \frac{3}{2}$ field. All vertices with $k_{\min }, k_{\max }$ as in (7.34) are candidates for the Yang-Mills interaction of the fermionic spin $s+\frac{1}{2} \geq \frac{3}{2}$ field. We impose an additional requirement, which allows us to choose one suitable vertex: given value of $s$, we look for the vertex with the minimal value of $k_{\max }$. It is easy to see that such a vertex is given by $k_{\max }=s+1$. Plugging $k_{\min }=0$, $k_{\max }=s+1$ into (7.23), (7.25), we get the Yang-Mills interaction of the massive spin $s+\frac{1}{2} \geq \frac{3}{2}$ field. 12

$$
p_{[3]}^{-}\left(s+\frac{1}{2}, s+\frac{1}{2}, 1 ; 0, s\right)=F_{0}^{(3)}\left(Q^{(12)}\right)^{s}, \quad s \geq 1 .
$$

A few remarks are in order.

i) The prefactor $F_{0}^{(3)}(7.13)$ has smooth massless limit $(\mathrm{m} \rightarrow 0)$. Therefore, the Yang-Mills interaction of the massive spin- $\frac{1}{2}$ fermionic field given in (7.35) has a smooth massless limit, as this should be. This interaction in the massless limit coincides with the respective interaction of the massless spin- $\frac{1}{2}$ fermionic field in Table I.

ii) The form $Q^{(12)}$ (7.17) does not have a smooth massless limit $(\mathrm{m} \rightarrow 0)$. This implies that the Yang-Mills interaction of the massive spin $s+\frac{1}{2}>\frac{3}{2}$ field (7.36) does not admit the massless limit. In the light-cone approach, it is the contribution of $Q^{(12)}$ that explains why the Yang-Mills interaction of the massive spin $s+\frac{1}{2}>\frac{3}{2}$ field does not admit the massless limit. As was expected, the Yang-Mills interaction of the massive spin $s+\frac{1}{2}>\frac{3}{2}$ field (7.36) involves higher derivatives. The appearance of the higher derivatives in (7.36) can be seen from the expression for $Q^{(12)}(7.17)$.

\subsubsection{Gravitational interaction of massive arbitrary spin fermionic field}

We now apply our results in Section 7.1.1 to discuss the gravitational interaction of massive arbitrary spin fermionic field. We first present the list of all cubic F-vertices for the massive spin- $\left(s+\frac{1}{2}\right)$ fermionic field interacting with massless spin-2 field. This is, we consider F-vertices (7.23) with

$$
\begin{array}{lll}
\mathrm{m}_{1}=\mathrm{m}, & \mathrm{m}_{2}=\mathrm{m}, & \mathrm{m}_{3}=0, \\
s^{(1)}+\frac{1}{2}, & s^{(2)}+\frac{1}{2}, & s^{(3)}=2,
\end{array}
$$

where $\mathrm{m} \neq 0, s^{(1)}=s^{(2)}=s$. The 2 nd restrictions in (7.28) lead to two allowed values of $k_{\min }$ : $k_{\text {min }}=0,1$. Plugging these values of $k_{\min }$ into the 1 st restrictions in (7.28), we obtain two families of F-vertices

$$
\begin{array}{lll}
k_{\min }=1, & s+2 \leq k_{\max } \leq 2 s+2, & s \geq 0 ; \\
k_{\min }=0, & s+1 \leq k_{\max } \leq 2 s, & s \geq 1 ;
\end{array}
$$

\footnotetext{
${ }^{12}$ A gauge invariant description of the electromagnetic interaction of the massive spin-2 field was obtained in Ref.[39]. The derivation of the electromagnetic interaction of massive spin $s=2,3$ fields from string theory is given in Refs. [40]. In these references, the electromagnetic field is treated as an external (non-dynamical) field.
} 
We now discuss those vertices in (7.38), (7.39) that correspond to the gravitational interaction of the massive arbitrary spin fermionic fields. We consider various spin fields in turn.

a) Spin- $\frac{1}{2}$ field $(s=0)$. The gravitational interaction of the massive spin- $\frac{1}{2}$ fermionic field is given by (7.38). Plugging $s=0$ in (7.38), we obtain the well-known relation $k_{\max }=2$, which tells us that the cubic vertex of the gravitational interaction of the massive spin- $\frac{1}{2}$ fermionic field is a degree-2 non-homogeneous polynomial in the derivatives. Formulas (7.23),(7.25) lead to the gravitational interaction of the massive spin- $\frac{1}{2}$ fermionic field,

$$
p_{[3]}^{-}\left(\frac{1}{2}, \frac{1}{2}, 2 ; 1,2\right)=F_{0}^{(3)} B^{(3)} .
$$

b) Spin $\frac{3}{2}$ field $(s=1)$. The gravitational interaction vertex of the massive spin- $\frac{3}{2}$ fermionic field is given in (7.39). Plugging $s=1$ in (7.39), we obtain $k_{\max }=2$. Formulas (7.23), (7.25) then lead to the gravitational interaction of the massive spin- $\frac{3}{2}$ field

$$
p_{[3]}^{-}\left(\frac{3}{2}, \frac{3}{2}, 2 ; 0,2\right)=F_{0}^{(3)} Z \text {. }
$$

c) Higher-spin fermionic fields, $\left(s+\frac{1}{2}\right) \geq \frac{5}{2}$. All vertices given in (7.38),(7.39) are candidates for the gravitational interaction of the higher-spin fermionic fields. We should impose some additional requirement that would allow us to choose one suitable vertex. Our additional requirement is that we look for vertex that has the minimal value of $k_{\max }$. It can be seen that such a vertex is given by (7.39) with $k_{\min }=0, k_{\max }=s+1$. Plugging such $k_{\min }, k_{\max }$ into (7.23), (7.25) we get cubic vertex for the gravitational interaction of the massive higher-spin fermionic field,

$$
p_{[3]}^{-}\left(s+\frac{1}{2}, s+\frac{1}{2}, 2 ; 0, s+1\right)=F_{0}^{(3)} Z\left(Q^{(12)}\right)^{s-1}, \quad s \geq 2 .
$$

A few remarks are in order.

i) Since prefactor $F_{0}^{(3)}(7.13)$, and the forms $B^{(3)}, Z$ (7.16), (7.18) have a smooth massless limit, $\mathrm{m} \rightarrow 0$, the gravitational interactions of the massive spin $-\frac{1}{2},-\frac{3}{2}$ fermionic fields (7.40), (7.41) have smooth massless limit, as they should. These gravitational interactions in the massless limit reduce to the corresponding interactions of the massless spin- $\frac{1}{2},-\frac{3}{2}$ fermionic fields given in Table I.

ii) Since the form $Q^{(12)}(7.17)$ does not have a smooth massless limit $(\mathrm{m} \rightarrow 0)$, the gravitational interaction of the massive higher-spin fermionic field (7.42) does not admit a sensible massless limit; it is the form $Q^{(12)}$ that explains why the gravitational interaction of the massive higher-spin field does not admit the massless limit. Higher derivatives in the gravitational interaction of the massive higher-spin fermionic field are related to the contribution of $Q^{(12)}(7.17) .13$

\subsubsection{Interaction of arbitrary spin massless bosonic field with two massive spin- $\frac{1}{2}$ fermionic fields}

Using results in Section 7.1.1, we now discuss the interaction vertices for massless arbitrary spin$s$ bosonic field and massive spin- $\frac{1}{2}$ fermionic fields 14 This is, we consider K- and F-vertices (7.22),(7.23) with the spin and mass values given by

$$
\begin{array}{lll}
\mathrm{m}_{1}=\mathrm{m}, & \mathrm{m}_{2}=\mathrm{m}, & \mathrm{m}_{3}=0, \\
s^{(1)}+\frac{1}{2}, & s^{(2)}+\frac{1}{2}, & s^{(3)}=s,
\end{array}
$$

\footnotetext{
${ }^{13}$ Gauge invariant formulations of the gravitational interaction of massive fields are studied e.g. in Ref.[41]. Interesting discussion of various aspects of the massive spin 2 field in gravitational background may be found in Refs. [42].

14 Discussion of vertices for arbitrary spin massless bosonic field and two massive scalar fields in framework of BRST invariant approach may be found in Ref.[43].
} 
where $\mathrm{m} \neq 0, s^{(1)}=s^{(2)}=0$. Plugging these spin values into (7.27), (7.28), we find that $k_{\min }$ and $k_{\max }$ are fixed uniquely and therefore there are only one allowed K-vertex and only one allowed F-vertex,

$$
\begin{array}{lll}
k_{\min }=s, & k_{\max }=s+1, & \text { for K-vertices, } \\
k_{\min }=s-1, & k_{\max }=s, & \text { for F-vertices. }
\end{array}
$$

Plugging (7.44) and (7.45) into (7.22)-(7.25) we obtain the respective K- and F- cubic interaction vertices

$$
\begin{aligned}
& p_{[3]}^{-}\left(\frac{1}{2}, \frac{1}{2}, s ; s, s+1\right)=K^{(12)}\left(B^{(3)}\right)^{s}, \\
& p_{[3]}^{-}\left(\frac{1}{2}, \frac{1}{2}, s ; s-1, s\right)=F_{0}^{(3)}\left(B^{(3)}\right)^{s-1} .
\end{aligned}
$$

Vertices (7.46), (7.47) have smooth massless limit.

\section{Cubic vertices for one massless fermionic field, one mas- sive fermionic field and one bosonic field with the same mass value}

As before, we start with vertices for mixed-symmetry fields and consider the cubic interaction vertices for one massless fermionic field and two massive fields (bosonic and fermionic) having the same mass values,

$$
\left.\mathrm{m}_{1}\right|_{B}=\left.\mathrm{m}_{2}\right|_{F} \equiv \mathrm{m} \neq 0,\left.\quad \mathrm{~m}_{3}\right|_{F}=0,
$$

i.e. the massive bosonic field carries external line index $a=1$, one massive fermionic field carries external line index $a=2$, while the massless fermionic field corresponds to $a=3$. We find the following solution for K- and F-vertices:

$$
\begin{array}{ll}
p_{[3]}^{-}=K^{(23)} V^{\mathrm{K}}\left(L_{n}^{(1)}, L_{n}^{(2)}, B_{n}^{(3)} ; Q_{m n}^{(12)} ; Z_{m n q}\right), & \text { K-vertex; } \\
p_{[3]}^{-}=F_{n} V^{\mathrm{F}}\left(L_{n}^{(1)}, L_{n}^{(2)}, B_{n}^{(3)} ; Q_{m n}^{(12)} ; Z_{m n q}\right), & \text { F-vertex; }
\end{array}
$$

where generating functions $V^{\mathrm{K}}, V^{\mathrm{F}}$ are arbitrary polynomials of linear, quadratic, and cubic forms. We use the notation

$$
\begin{aligned}
& K^{(23)}=\frac{1}{\beta_{2} \beta_{3}} p_{\theta_{2}}\left(\mathbb{P}^{I} \gamma^{I} \gamma_{*}-\mathrm{m} \beta_{3}\right) \eta_{3} \\
& F_{n}= \frac{1}{\beta_{2} \beta_{3}} p_{\theta_{2}}\left(\frac{\check{\beta}_{1}}{\beta_{1}} \mathbb{P}^{I}-\gamma^{I J} \mathbb{P}^{J}+\mathrm{m} \beta_{3} \gamma^{I} \gamma_{*}\right) \eta_{3} \alpha_{n}^{(1) I} \\
&+\frac{1}{\beta_{2} \beta_{3}} p_{\theta_{2}}\left(-\mathbb{P}^{I} \gamma^{I} \gamma_{*}+\frac{\check{\beta}_{1}}{\beta_{1}} \beta_{3} \mathrm{~m}\right) \eta_{3} \zeta_{n}^{(1)}, \\
& L_{n}^{(1)} \equiv B_{n}^{(1)}-\frac{1}{2} \mathrm{~m} \zeta_{n}^{(1)}, \quad L_{n}^{(2)} \equiv B_{n}^{(2)}+\frac{1}{2} \mathrm{~m} \zeta_{n}^{(2)}
\end{aligned}
$$




$$
\begin{aligned}
& B_{n}^{(a)} \equiv \frac{\alpha_{n}^{(a) I} \mathbb{P}^{I}}{\beta_{a}}-\frac{\check{\beta}_{a}}{2 \beta_{a}} \mathrm{~m} \zeta_{n}^{(a)}, \quad a=1,2 ; \\
& B_{n}^{(3)} \equiv \frac{\alpha_{n}^{(3) I} \mathbb{P}^{I}}{\beta_{3}} \\
& Q_{m n}^{(12)} \equiv \alpha_{m n}^{(12)}-\frac{\zeta_{n}^{(2)}}{\mathrm{m}} B_{m}^{(1)}+\frac{\zeta_{m}^{(1)}}{\mathrm{m}} B_{n}^{(2)}, \\
& Z_{m n q} \equiv L_{m}^{(1)} \alpha_{n q}^{(23)}+L^{(2)} \alpha^{(31)}+B_{q}^{(3)}\left(\alpha_{m n}^{(12)}-\zeta_{m}^{(1)} \zeta_{n}^{(2)}\right),
\end{aligned}
$$

where the quadratic forms $\alpha_{m n}^{(a b)}$ are defined in (4.8).

Comparing vertices (8.2), (8.3) with the ones in (7.2), (7.3), we see that generating functions $V^{\mathrm{K}}, V^{\mathrm{F}}$ given in (8.2), (8.3) coincide with ones given in (7.2), (7.3) in Section 7 . Vertices in (8.2), (8.3) and the ones in (7.2), (7.3) are distinguished only by prefactors given in (8.4), (8.5) and (7.4), (7.5) respectively. Note however that, as in Section 7 , the prefactors in (8.4), (8.5) are also non-homogeneous polynomials in $\mathbb{P}^{I}$. This implies that, in general, cubic interaction vertices for fields with mass parameters as in (8.1) are also non-homogeneous polynomials in $\mathbb{P}^{I}$. As before, solution for $V^{\mathrm{K}}, V^{\mathrm{F}}$ given in (8.2), (8.3) is the complete solution, while the solution for the prefactors $K^{(23)}, F_{n}$ given in (8.4), (8.5) is not complete solution. Namely, for the vertices of mixed-symmetry fields there are extra solutions for the prefactors that involve contributions of higher than second order in $\gamma$-matrices.

To discuss the remaining important properties of solution $(8.2),(8.3)$ we restrict our attention to cubic vertices for totally symmetric fields.

\subsection{Cubic vertices for totally symmetric fields}

In this section, we study vertices for three totally symmetric fields with the mass values given in (8.1), i.e. the massive bosonic field carries external line index $a=1$, one massive fermionic field carries external line index $a=2$, while the massless fermionic field corresponds to $a=3$. Because for the studying the totally symmetric fields it is sufficient to use one sort of oscillators, we set $\nu=1$ in (8.2), (8.3) and ignore the contribution of the oscillators with $n>1$. To simplify the formulas we drop the oscillator's subscript $n=1$ and use the simplified notation for oscillators: $\alpha^{(a) I} \equiv \alpha_{1}^{(a) I}, \zeta^{(a)} \equiv \zeta_{1}^{(a)}$. In doing so, we obtain, using the solution given in (8.2), (8.3), the cubic interaction vertices for totally symmetric fields,

$$
\begin{array}{ll}
p_{[3]}^{-}=K^{(23)} V^{\mathrm{K}}\left(L^{(1)}, L^{(2)}, B^{(3)} ; Q^{(12)} ; Z\right), & \text { K-vertex; } \\
p_{[3]}^{-}=F V^{\mathrm{F}}\left(L^{(1)}, L^{(2)}, B^{(3)} ; Q^{(12)} ; Z\right), & \text { F-vertex; }
\end{array}
$$

where we use the notation

$$
\begin{aligned}
& K^{(23)} \equiv \frac{1}{\beta_{2} \beta_{3}} p_{\theta_{2}}\left(\mathbb{P}^{I} \gamma^{I} \gamma_{*}-\mathrm{m} \beta_{3}\right) \eta_{3} \\
& F \equiv \frac{1}{\beta_{2} \beta_{3}} p_{\theta_{2}}\left(\frac{\check{\beta}_{1}}{\beta_{1}} \mathbb{P}^{I}-\gamma^{I J} \mathbb{P}^{J}+\mathrm{m} \beta_{3} \gamma^{I} \gamma_{*}\right) \eta_{3} \alpha^{(1) I} \\
& \quad+\frac{1}{\beta_{2} \beta_{3}} p_{\theta_{2}}\left(-\mathbb{P}^{I} \gamma^{I} \gamma_{*}+\frac{\check{\beta}_{1}}{\beta_{1}} \beta_{3} \mathrm{~m}\right) \eta_{3} \zeta^{(1)}
\end{aligned}
$$




$$
\begin{aligned}
& L^{(1)} \equiv B^{(1)}-\frac{1}{2} \mathrm{~m} \zeta^{(1)}, \quad L^{(2)} \equiv B^{(2)}+\frac{1}{2} \mathrm{~m} \zeta^{(2)} \\
& B^{(a)} \equiv \frac{\alpha^{(a) I} \mathbb{P}^{I}}{\beta_{a}}-\frac{\check{\beta}_{a}}{2 \beta_{a}} \mathrm{~m} \zeta^{(a)}, \quad a=1,2 ; \\
& B^{(3)} \equiv \frac{\alpha^{(3) I} \mathbb{P}^{I}}{\beta_{3}}, \\
& Q^{(12)} \equiv \alpha^{(12)}-\frac{\zeta^{(2)}}{\mathrm{m}} B^{(1)}+\frac{\zeta^{(1)}}{\mathrm{m}} B^{(2)}, \\
& Z \equiv L^{(1)} \alpha^{(23)}+L^{(2)} \alpha^{(31)}+B^{(3)}\left(\alpha^{(12)}-\zeta^{(1)} \zeta^{(2)}\right) .
\end{aligned}
$$

Solution in (8.11), (8.12) is the complete solution, i.e., vertices given in $(8.11),(8.12)$ provide the complete list of parity invariant cubic vertices for the totally symmetric fields with the mass parameters given in (8.1). To discuss the remaining important properties of solution in (8.11), (8.12) we restrict our attention to cubic vertices for totally symmetric fields with fixed spin values.

\subsubsection{Cubic vertices for totally symmetric fields with fixed but arbitrary spin values}

We now restrict ourselves to cubic vertices for the totally symmetric fields with fixed but arbitrary spin values and with mass values given in (8.1). Vertices $8.11,(8.12)$ describe interaction of the towers of fermionic and bosonic fields. We next consider the vertices involving one massive spin$s^{(1)}$ bosonic field, one massive spin- $\left(s^{(2)}+\frac{1}{2}\right)$ fermionic field and one massless spin- $\left(s^{(3)}+\frac{1}{2}\right)$ fermionic field, where bosonic and fermionic fields have the same mass parameter $m \neq 0$ :

$$
\begin{array}{lll}
\mathrm{m}_{1}=\mathrm{m}, & \mathrm{m}_{2}=\mathrm{m}, & \mathrm{m}_{3}=0, \\
s^{(1)}, & s^{(2)}+\frac{1}{2}, & s^{(3)}+\frac{1}{2} .
\end{array}
$$

Massive spin- $s^{(1)}$ bosonic field and massive spin- $\left(s^{(2)}+\frac{1}{2}\right)$ fermionic field are described by the respective ket-vectors $\left|\phi_{s^{(1)}}\right\rangle$ and $\left|\psi_{s^{(2)}}\right\rangle$, while the massless spin- $\left(s^{(3)}+\frac{1}{2}\right)$ fermionic field is described by ket-vector $\left|\psi_{s^{(3)}}\right\rangle$. The ket-vectors $\left|\phi_{s^{(1)}}\right\rangle,\left|\psi_{s^{(2)}}\right\rangle$ can be obtained from the respective expressions in (2.11), (2.23) by the replacement $s \rightarrow s^{(a)}, \alpha^{I} \rightarrow \alpha^{(a) I}, \zeta \rightarrow \zeta^{(a)}, a=1$, 2, while the ket-vector $\left|\psi_{s^{(3)}}\right\rangle$ can be obtained from (2.23) by the replacement $s \rightarrow s^{(3)}, \alpha^{I} \rightarrow \alpha^{(3) I}$. Taking into account that the ket-vectors $\left|\phi_{s^{(1)}}\right\rangle,\left|\psi_{s^{(2)}}\right\rangle$ are the respective degree- $s^{(a)}$ homogeneous polynomials in the oscillators $\alpha^{(a) I}, \zeta^{(a)}, a=1,2$, while the ket-vector $\left|\psi_{s}(3)\right\rangle$ is a degree-s $s^{(3)}$ homogeneous polynomial in the oscillator $\alpha^{(3) I}$, we see that the vertices we are interested in must satisfy the equations

$$
\begin{aligned}
& \left(\alpha^{(a) I} \bar{\alpha}^{(a) I}+\zeta^{(a)} \bar{\zeta}^{(a)}-s^{(a)}\right)\left|p_{[3]}^{-}\right\rangle=0, \quad a=1,2, \\
& \left(\alpha^{(3) I} \bar{\alpha}^{(3) I}-s^{(3)}\right)\left|p_{[3]}^{-}\right\rangle=0 .
\end{aligned}
$$

These equations tell us that the vertices must be a degree- $s^{(a)}$ homogeneous polynomial in the respective oscillators. Taking into account that the prefactor $F$ and the forms $L^{(1)}, L^{(2)}, B^{(3)}(8.14)$ (8.17) are degree- 1 homogeneous polynomials in the oscillators, while forms $Q^{(12)}, Z,(8.18$, (8.19) are the respective degree- 2 and degree- 3 homogeneous polynomials in the oscillators we find the general solution of Eqs. (8.21), (8.22) as

$$
p_{[3]}^{-}\left(s^{(1)}, s^{(2)}+\frac{1}{2}, s^{(3)}+\frac{1}{2} ; k_{\min }, k_{\max }\right)
$$




$$
\begin{array}{llr}
=K^{(23)}\left(L^{(1)}\right)^{w^{(1)}}\left(L^{(2)}\right)^{w^{(2)}}\left(B^{(3)}\right)^{w^{(3)}}\left(Q^{(12)}\right)^{y^{(3)}} Z^{y}, & & \text { K-vertex; } \\
=F\left(L^{(1)}\right)^{x^{(1)}}\left(L^{(2)}\right)^{x^{(2)}}\left(B^{(3)}\right)^{x^{(3)}}\left(Q^{(12)}\right)^{y^{(3)}} Z^{y}, & & \text { F-vertex },
\end{array}
$$

where the parameters $w^{(1)}, w^{(2)}, w^{(3)}, y^{(3)}, y$ are given by

$$
\begin{array}{ll}
w^{(1)}=k_{\max }-k_{\min }-s^{(2)}-1, & \\
w^{(2)}=k_{\max }-k_{\min }-s^{(1)}-1, & \\
w^{(3)}=k_{\min } & \\
y^{(3)}=\mathbf{s}-2 s^{(3)}-k_{\max }+2 k_{\min }+1, & \\
y=s^{(3)}-k_{\min }, & \\
w^{(1)}=k_{\max }-k_{\min }-s^{(2)}-1, & \\
w^{(2)}=k_{\max }-k_{\min }-s^{(1)}, & \\
w^{(3)}=k_{\min }, & \\
y^{(3)}=\mathbf{s}-2 s^{(3)}-k_{\max }+2 k_{\min }, & \\
y=s^{(3)}-k_{\min }, & \text { for F-vertices; }
\end{array}
$$

and $\mathrm{s}$ is defined in (4.22). Integers $k_{\min }$ and $k_{\max }$ in (8.23)-(8.26) are the freedom in our solution. In general, vertices (8.23), (8.24) are non-homogeneous polynomials in the momentum $\mathbb{P}^{I}$ and the integers $k_{\min }$ and $k_{\max }$ are the respective minimal and maximal numbers of powers of the momentum $\mathbb{P}^{I}$ in (8.23), 8.24). For vertices (8.23), (8.24) to be sensible, we should impose the restrictions

$$
x^{(a)} \geq 0, \quad a=1,2,3 ; \quad y^{(3)} \geq 0, \quad y \geq 0,
$$

which amount to requiring the powers of all forms in 8.23 , (8.24) to be non-negative integers. Using (8.25), (8.26), restrictions (8.27) can be rewritten in a more convenient form as

$$
\begin{array}{ll}
k_{\min }+\max _{a=1,2} s^{(a)}+1 \leq k_{\max } \leq \mathbf{s}-2 s^{(3)}+2 k_{\min }+1, & \text { for K-vertices; } \\
0 \leq k_{\min } \leq s^{(3)}, & \text { for F-vertices } . \\
k_{\min }+\max \left(s^{(1)}-1, s^{(2)}\right)+1 \leq k_{\max } \leq \mathbf{s}-2 s^{(3)}+2 k_{\min }, \\
0 \leq k_{\min } \leq s^{(3)},
\end{array}
$$

Cubic vertices in (8.23), (8.24) and restrictions on the allowed values of $k_{\max }$ and $k_{\min }$ in (8.28), (8.29) provide the complete list of parity invariant cubic vertices for the totally symmetric fields with spin and mass values given in (8.20).

\section{Cubic vertices for one massless bosonic field and two massive fermionic fields with different mass values}

We now consider the cubic vertices for mixed-symmetry fields with the following mass values:

$$
\left.\mathrm{m}_{1}\right|_{F} \neq 0,\left.\quad \mathrm{~m}_{2}\right|_{F} \neq 0, \quad \mathrm{~m}_{1} \neq \mathrm{m}_{2},\left.\quad \mathrm{~m}_{3}\right|_{B}=0,
$$


i.e. the massive fermionic fields carry external line indices $a=1,2$, while the massless bosonic field corresponds to $a=3$. Equations for the vertex involving one massless field can be obtained from Eqs. (3.31) in the limit as $\mathrm{m}_{3} \rightarrow 0$. The $\mathrm{K}$ - and F-vertices we found take the form

$$
\begin{array}{ll}
p_{[3]}^{-}=K^{(12)} V^{\mathrm{K}}\left(L_{n}^{(1)}, L_{n}^{(2)} ; Q_{m n}^{(a a+1)}\right), & \text { K-vertex; } \\
p_{[3]}^{-}=F_{n} V^{\mathrm{F}}\left(L_{n}^{(1)}, L_{n}^{(2)} ; Q_{m n}^{(a a+1)}\right), & \text { F-vertex; }
\end{array}
$$

where generating functions $V^{\mathrm{K}}, V^{\mathrm{F}}$ are arbitrary polynomials of linear and quadratic forms. We use the notation

$$
\begin{aligned}
K^{(12)} \equiv \frac{1}{\beta_{1} \beta_{2}} p_{\theta_{1}}\left(\mathbb{P}^{I} \gamma^{I} \gamma_{*}-\mathrm{m}_{1} \beta_{2}+\mathrm{m}_{2} \beta_{1}\right) \eta_{2} \\
F_{n} \equiv \frac{1}{\beta_{1} \beta_{2}} p_{\theta_{1}}\left(\frac{\check{\beta}_{3}}{\beta_{3}} \mathbb{P}^{I}-\gamma^{I J} \mathbb{P}^{J}+\left(\mathrm{m}_{1} \beta_{2}+\mathrm{m}_{2} \beta_{1}\right) \gamma^{I} \gamma_{*}\right) \eta_{2} \alpha_{n}^{(3) I}-\frac{2}{\mathrm{~m}_{1}+\mathrm{m}_{2}} K^{(12)} B_{n}^{(3)} \\
L_{n}^{(1)} \equiv B_{n}^{(1)}-\frac{\mathrm{m}_{2}^{2}}{2 \mathrm{~m}_{1}} \zeta^{(1)}, \quad L_{n}^{(2)} \equiv B_{n}^{(2)}+\frac{\mathrm{m}_{1}^{2}}{2 \mathrm{~m}_{2}} \zeta_{n}^{(2)} \\
B_{n}^{(a)} \equiv \frac{\alpha_{n}^{(a) I} \mathbb{P}^{I}}{\beta_{a}}-\frac{\check{\beta}_{a}}{2 \beta_{a}} \mathrm{~m}_{a} \zeta_{n}^{(a)}, \\
B_{n}^{(3)} \equiv \frac{\alpha_{n}^{(3) I} \mathbb{P}^{I}}{\beta_{3}}, \\
Q_{m n}^{(12)} \equiv \alpha_{m n}^{(12)}-\frac{\zeta_{n}^{(2)}}{\mathrm{m}_{2}} B_{m}^{(1)}+\frac{\zeta_{m}^{(1)}}{\mathrm{m}_{1}} B_{n}^{(2)} \\
Q_{m n}^{(23)} \equiv \alpha_{m n}^{(23)}-\frac{\mathrm{m}_{2} \zeta_{m}^{(2)}}{\mathrm{m}_{1}^{2}-\mathrm{m}_{2}^{2}} B_{n}^{(3)}-\frac{2}{\mathrm{~m}_{1}^{2}-\mathrm{m}_{2}^{2}} B_{m}^{(2)} B_{n}^{(3)} \\
Q_{m n}^{(31)} \equiv \alpha_{m n}^{(31)}-\frac{\mathrm{m}_{1} \zeta_{n}^{(1)}}{\mathrm{m}_{1}^{2}-\mathrm{m}_{2}^{2}} B_{m}^{(3)}+\frac{2}{\mathrm{~m}_{1}^{2}-\mathrm{m}_{2}^{2}} B_{m}^{(3)} B_{n}^{(1)}
\end{aligned}
$$

and the quadratic forms and $\alpha_{m n}^{(a b)}$ are defined in (4.8).

The following remarks are in order.

i) We note appearance of expressions like $\mathrm{m}_{1}^{2}-\mathrm{m}_{2}^{2}$ in the denominators of quadratic forms $Q_{m n}^{(23)}$, $Q_{m n}^{(31)}$, 9.10), (9.11), i.e., we see that the quadratic forms $Q_{m n}^{(23)}, Q_{m n}^{(31)}$ are singular as $\mathrm{m}_{1} \rightarrow \mathrm{m}_{2}$. For this reason, we considered the case of $\mathrm{m}_{1}=\mathrm{m}_{2}$ separately in Section 7 .

ii) From (9.4)-(9.11), we see that the prefactors $K^{(12)}, F_{n}$, the linear forms $L_{n}^{(1)}, L_{n}^{(2)}$, and the quadratic forms $Q_{m n}^{(a a+1)}$ are non-homogeneous polynomials in $\mathbb{P}^{I}$. This implies that, in general, the cubic vertices given in (9.2), (9.3) are a non-homogeneous polynomials in $\mathbb{P}^{I}$.

iii) Solution for generating functions $V^{\mathrm{K}}, V^{\mathrm{F}}$ given in (9.2), (9.3) is complete solution, while the solution for the prefactors $K^{(12)}, F_{n}$ given in (9.4), (9.5) is not complete solution. Namely, for the vertices of mixed-symmetry fields there are extra solutions for the prefactors that involve contribution of higher than second order in $\gamma$-matrices.

To understand the remaining characteristic properties of the solution obtained, we consider the vertices for totally symmetric fields. 


\subsection{Cubic vertices for totally symmetric one massless bosonic field and two massive fermionic fields with different mass values}

We proceed with the study of vertices for three totally symmetric fields with the mass values given in (9.1), i.e. the massive fermionic fields carry external line indices $a=1$, 2 , while the massless bosonic field corresponds to $a=3$. Since for the studying the totally symmetric fields it is sufficient to use one sort of oscillators, we set $\nu=1$ in (9.2), (9.3) and ignore the contribution of the oscillators with $n>1$. To simplify the formulas we drop the oscillator's subscript $n=1$ and use the simplified notation for oscillators: $\alpha^{(a) I} \equiv \alpha_{1}^{(a) I}, \zeta^{(a)} \equiv \zeta_{1}^{(a)}$. In doing so, we obtain, using the solution given in (9.2), (9.3), the cubic interaction vertices for totally symmetric fields,

$$
\begin{array}{ll}
p_{[3]}^{-}=K^{(12)} V^{\mathrm{K}}\left(L^{(1)}, L^{(2)} ; Q^{(a a+1)}\right), & \text { K-vertex; } \\
p_{[3]}^{-}=F V^{\mathrm{F}}\left(L^{(1)}, L^{(2)} ; Q^{(a a+1)}\right), & \text { F-vertex; }
\end{array}
$$

where we use the notation

$$
\begin{gathered}
F \equiv \frac{1}{\beta_{1} \beta_{2}} p_{\theta_{1}}\left(\frac{\check{\beta}_{3}}{\beta_{3}} \mathbb{P}^{I}-\gamma^{I J} \mathbb{P}^{J}+\left(\mathrm{m}_{1} \beta_{2}+\mathrm{m}_{2} \beta_{1}\right) \gamma^{I} \gamma_{*}\right) \eta_{2} \alpha^{(3) I}-\frac{2}{\mathrm{~m}_{1}+\mathrm{m}_{2}} K^{(12)} B^{(3)} \\
L^{(1)} \equiv B^{(1)}-\frac{\mathrm{m}_{2}^{2}}{2 \mathrm{~m}_{1}} \zeta^{(1)}, \quad L^{(2)} \equiv B^{(2)}+\frac{\mathrm{m}_{1}^{2}}{2 \mathrm{~m}_{2}} \zeta^{(2)} \\
B^{(a)} \equiv \frac{\alpha^{(a) I} \mathbb{P}^{I}}{\beta_{a}}-\frac{\check{\beta}_{a}}{2 \beta_{a}} \mathrm{~m}_{a} \zeta^{(a)}, \quad a=1,2 \\
B^{(3)} \equiv \frac{\alpha^{(3)} I \mathbb{P}^{I}}{\beta_{3}}, \\
Q^{(12)} \equiv \alpha^{(12)}-\frac{\zeta^{(2)}}{\mathrm{m}_{2}} B^{(1)}+\frac{\zeta^{(1)}}{\mathrm{m}_{1}} B^{(2)}, \\
Q^{(23)} \equiv \alpha^{(23)}-\frac{\mathrm{m}_{2} \zeta^{(2)}}{\mathrm{m}_{1}^{2}-\mathrm{m}_{2}^{2}} B^{(3)}-\frac{2}{\mathrm{~m}_{1}^{2}-\mathrm{m}_{2}^{2}} B^{(2)} B^{(3)} \\
Q^{(31)} \equiv \alpha^{(31)}-\frac{\mathrm{m}_{1} \zeta^{(1)}}{\mathrm{m}_{1}^{2}-\mathrm{m}_{2}^{2}} B^{(3)}+\frac{2}{\mathrm{~m}_{1}^{2}-\mathrm{m}_{2}^{2}} B^{(3)} B^{(1)}
\end{gathered}
$$

The quadratic forms $\alpha^{(a b)}$ and prefactor $K^{(12)}$ are defined in (4.17) and (9.4) respectively. Solution given in (9.12), (9.13) is the complete solution. In other words, solution given in (9.12), (9.13) provides the complete list of parity invariant cubic interaction for totally symmetric field with mass parameters given in (9.1). To understand the remaining characteristic properties of the solution obtained, we consider the vertices for the totally symmetric fields with fixed spin values.

\subsubsection{Cubic vertices for totally symmetric fields with fixed but arbitrary mass values}

Discussion of cubic interaction vertices for two massive fermionic fields with different mass values and one massless bosonic field is similar the one in Section 7 , This, we consider vertices involving 
two massive spin- $\left(s^{(1)}+\frac{1}{2}\right)$ and spin- $\left(s^{(2)}+\frac{1}{2}\right)$ fermionic fields and one massless spin- $s^{(3)}$ bosonic field, where the fermionic fields have different values of the mass parameter:

$$
\begin{array}{lll}
\mathrm{m}_{1} \neq 0, & \mathrm{~m}_{2} \neq 0, \quad \mathrm{~m}_{1} \neq \mathrm{m}_{2}, & \mathrm{~m}_{3}=0, \\
s^{(1)}+\frac{1}{2}, & s^{(2)}+\frac{1}{2}, & s^{(3)} .
\end{array}
$$

The vertices for totally symmetric spin- $\left(s^{(1)}+\frac{1}{2}\right)$ and spin- $\left(s^{(2)}+\frac{1}{2}\right)$ fermionic fields $\left|\psi_{s^{(1)}}\right\rangle,\left|\psi_{s^{(2)}}\right\rangle$ with different mass values and one massless spin- $s^{(3)}$ bosonic field $\left|\phi_{s^{(3)}}\right\rangle$ can be obtained by solving Eqs.(7.20), (7.21) with $p_{[3]}^{-}$given in (9.12), (9.13). We then obtain the cubic vertices

$$
\begin{array}{rll}
p_{[3]}^{-}\left(s^{(1)}+\frac{1}{2}, s^{(2)}+\frac{1}{2}, s^{(3)} ; w^{(1)}, w^{(2)}\right) & \\
=K^{(12)}\left(L^{(1)}\right)^{w^{(1)}}\left(L^{(2)}\right)^{w^{(2)}} \prod_{a=1}^{3}\left(Q^{(a a+1)}\right)^{y^{(a+2)}}, & \text { K-vertex; } \\
=F\left(L^{(1)}\right)^{w^{(1)}}\left(L^{(2)}\right)^{w^{(2)}} \prod_{a=1}^{3}\left(Q^{(a a+1)}\right)^{y^{(a+2)}}, & \text { F-vertex; }
\end{array}
$$

where the parameters $y^{(a)}$ are given by

$$
\begin{array}{ll}
y^{(1)}=\frac{1}{2}\left(s^{(2)}+s^{(3)}-s^{(1)}+w^{(1)}-w^{(2)}\right), & \\
y^{(2)}=\frac{1}{2}\left(s^{(1)}+s^{(3)}-s^{(2)}-w^{(1)}+w^{(2)}\right), & \text { for K-vertex; } \\
y^{(3)}=\frac{1}{2}\left(s^{(1)}+s^{(2)}-s^{(3)}-w^{(1)}-w^{(2)}\right), & \\
y^{(1)}=\frac{1}{2}\left(s^{(2)}+s^{(3)}-s^{(1)}+w^{(1)}-w^{(2)}-1\right), & \\
y^{(2)}= & \frac{1}{2}\left(s^{(1)}+s^{(3)}-s^{(2)}-w^{(1)}+w^{(2)}-1\right), \\
y^{(3)}= & \frac{1}{2}\left(s^{(1)}+s^{(2)}-s^{(3)}-w^{(1)}-w^{(2)}+1\right),
\end{array}
$$

Two integers $w^{(1)}, w^{(2)}$ are the freedom of our solution. For fixed spin values $s^{(1)}, s^{(2)}, s^{(3)}$, these integers label all possible cubic interaction vertices that can be built for the fields under consideration. For vertices (9.22), (9.23) to be sensible we impose the restrictions

$$
\begin{array}{lll}
w^{(1)} \geq 0, \quad w^{(2)} \geq 0, \quad y^{(a)} \geq 0, & a=1,2,3 ; & \\
\mathbf{s}-w^{(1)}-w^{(2)} \quad \text { even integer }, & \text { for K-vertex; } \\
w^{(1)} \geq 0, \quad w^{(2)} \geq 0, \quad y^{(a)} \geq 0, & a=1,2,3 ; & \\
\mathbf{s}-w^{(1)}-w^{(2)} \text { odd integer }, & \text { for F-vertex }
\end{array}
$$

which amount to the requirement that the powers of all forms in (9.22), (9.23) be non-negative integers. The maximal and minimal number of powers of $\mathbb{P}^{I}$ in $(9.22),(9.23)$, which we denote by 
$k_{\max }$ and $k_{\min }$ respectively, are given by 15

$$
\begin{array}{lll}
k_{\max }=\frac{1}{2}\left(s^{(1)}+s^{(2)}+3 s^{(3)}+w^{(1)}+w^{(2)}\right)+1, & k_{\min }=0, & \text { for K-vertex; } \\
k_{\max }=\frac{1}{2}\left(s^{(1)}+s^{(2)}+3 s^{(3)}+w^{(1)}+w^{(2)}+1\right), & k_{\min }=0, & \text { for F-vertex. }
\end{array}
$$

We note that using (9.24), (9.25) allows rewriting restrictions (9.26), (9.27) in the equivalent form 16

$$
\begin{array}{ll}
\left|s^{(1)}-s^{(2)}-w^{(1)}+w^{(2)}\right| \leq s^{(3)} \leq s^{(1)}+s^{(2)}-w^{(1)}-w^{(2)}, & \text { for K-vertex; } \\
\left|s^{(1)}-s^{(2)}-w^{(1)}+w^{(2)}\right|+1 \leq s^{(3)} \leq s^{(1)}+s^{(2)}-w^{(1)}-w^{(2)}+1, & \text { for F-vertex; }
\end{array}
$$

\section{Cubic vertices for one massless fermionic field, one massive fermionic field and one massive bosonic field with different mass values}

We now consider the cubic interaction vertices for mixed-symmetry fields with the following mass values:

$$
\left.\mathrm{m}_{1}\right|_{B} \neq 0,\left.\quad \mathrm{~m}_{2}\right|_{F} \neq 0, \quad \mathrm{~m}_{1} \neq \mathrm{m}_{2},\left.\quad \mathrm{~m}_{3}\right|_{F}=0
$$

i.e. the massive bosonic field carries external line index $a=1$, the massive fermionic field carries external line index $a=2$, while the massless fermionic field corresponds to $a=3$. Equations for the vertex involving one massless field can be obtained from Eqs.(3.31) in the limit as $\mathrm{m}_{3} \rightarrow 0$. The solution for $\mathrm{K}$ - and F-vertices we found is given by

$$
\begin{array}{ll}
p_{[3]}^{-}=K^{(23)} V^{\mathrm{K}}\left(L_{n}^{(1)}, L_{n}^{(2)} ; Q_{m n}^{(a a+1)}\right), & \text { K-vertex; } \\
p_{[3]}^{-}=F_{n} V^{\mathrm{F}}\left(L_{n}^{(1)}, L_{n}^{(2)} ; Q_{m n}^{(a a+1)}\right), & \text { F-vertex; }
\end{array}
$$

where generating functions $V^{\mathrm{K}}, V^{\mathrm{F}}$ are arbitrary polynomials of linear and quadratic forms. We use the notation

$$
\begin{aligned}
K^{(23)} & \equiv \frac{1}{\beta_{2} \beta_{3}} p_{\theta_{2}}\left(\mathbb{P}^{I} \gamma^{I} \gamma_{*}-\mathrm{m}_{2} \beta_{3}\right) \eta_{3}, \\
F_{n} \equiv & \frac{1}{\beta_{2} \beta_{3}} p_{\theta_{2}}\left(\frac{\check{\beta}_{1}}{\beta_{1}} \mathbb{P}^{I}-\gamma^{I J} \mathbb{P}^{J}+\beta_{3} \mathrm{~m}_{2} \gamma^{I} \gamma_{*}\right) \eta_{3} \alpha_{n}^{(1) I} \\
& +\frac{1}{\beta_{2} \beta_{3}} p_{\theta_{2}}\left(-\mathrm{m}_{2} \mathbb{P}^{I} \gamma^{I} \gamma_{*}+\beta_{3} \mathrm{~m}_{2}^{2}+\frac{2 \beta_{2} \beta_{3}}{\beta_{1}} \mathrm{~m}_{1}^{2}\right) \eta_{3} \frac{\zeta_{n}^{(1)}}{\mathrm{m}_{1}} \\
L_{n}^{(1)} & \equiv B_{n}^{(1)}-\frac{\mathrm{m}_{2}^{2}}{2 \mathrm{~m}_{1}} \alpha_{n}^{(1)}, \quad L_{n}^{(2)} \equiv B_{n}^{(2)}+\frac{\mathrm{m}_{1}^{2}}{2 \mathrm{~m}_{2}} \zeta_{n}^{(2)},
\end{aligned}
$$

\footnotetext{
${ }^{15}$ Expressions for $K^{(12)}$ (9.4) and $F, L^{(a)}, Q^{(a a+1)}$ (9.14)-(9.20) imply that $k_{\max }=w^{(1)}+w^{(2)}+2 y^{(1)}+2 y^{(2)}+$ $y^{(3)}+1$ for K-vertex and $k_{\max }=w^{(1)}+w^{(2)}+2 y^{(1)}+2 y^{(2)}+y^{(3)}+2$ for F-vertex. Relations for $y^{(a)}$ (9.24), (9.25) then lead to $k_{\max }$ given in 9.28 , 9.29 .

${ }^{16}$ If $w^{(1)}=w^{(2)}=0$, then restrictions (9.30) becomes the restrictions well known in the angular momentum theory: $\left|s^{(1)}-s^{(2)}\right| \leq s^{(3)} \leq s^{(1)}+s^{(2)}$, while restriction (9.31) takes the form $\left|s^{(1)}-s^{(2)}\right| \leq s^{(3)}-1 \leq s^{(1)}+s^{(2)}$.
} 


$$
\begin{aligned}
B_{n}^{(a)} & \equiv \frac{\alpha_{n}^{(a) I} \mathbb{P}^{I}}{\beta_{a}}-\frac{\check{\beta}_{a}}{2 \beta_{a}} \mathrm{~m}_{a} \zeta_{n}^{(a)}, \quad a=1,2 \\
B_{n}^{(3)} & \equiv \frac{\alpha_{n}^{(3) I} \mathbb{P}^{I}}{\beta_{3}} \\
Q_{m n}^{(12)} & \equiv \alpha_{m n}^{(12)}-\frac{\zeta_{n}^{(2)}}{\mathrm{m}_{2}} B_{m}^{(1)}+\frac{\zeta_{m}^{(1)}}{\mathrm{m}_{1}} B_{n}^{(2)}, \\
Q_{m n}^{(23)} & \equiv \alpha_{m n}^{(23)}-\frac{\mathrm{m}_{2} \zeta_{m}^{(2)}}{\mathrm{m}_{1}^{2}-\mathrm{m}_{2}^{2}} B_{n}^{(3)}-\frac{2}{\mathrm{~m}_{1}^{2}-\mathrm{m}_{2}^{2}} B_{m}^{(2)} B_{n}^{(3)} \\
Q_{m n}^{(31)} & \equiv \alpha_{m n}^{(31)}-\frac{\mathrm{m}_{1} \zeta_{n}^{(1)}}{\mathrm{m}_{1}^{2}-\mathrm{m}_{2}^{2}} B_{m}^{(3)}+\frac{2}{\mathrm{~m}_{1}^{2}-\mathrm{m}_{2}^{2}} B_{m}^{(3)} B_{n}^{(1)}
\end{aligned}
$$

and the quadratic forms $\alpha_{m n}^{(a b)}$ are defined in (4.8). Comparing vertices (10.2),(10.3) with the ones in (9.2), (9.3), we see that generating functions $V^{\mathrm{K}}, V^{\mathrm{F}}$ given in (10.2),(10.3) coincide with the ones discussed in (9.2), (9.3) in Section 9. Vertices in (10.2), (10.3) and the ones in (9.2), (9.3) are distinguished only by the prefactors given in (10.4), (10.5) and (9.4), (9.5) respectively. Note however that, as in Section 9, the prefactors in (10.4), (10.5) are also non-homogeneous polynomials in $\mathbb{P}^{I}$. This implies that, in general, cubic vertices for fields with mass parameters given in (10.1) are also non-homogeneous polynomials in $\mathbb{P}^{I}$. As before, solution for $V^{\mathrm{K}}, V^{\mathrm{F}}$ given in (10.2), (10.3) is complete solution, while the solution for the prefactors $K^{(23)}, F_{n}$ given in (10.4), (10.5) is not complete solution. Namely, for the vertices of mixed-symmetry fields there are extra solutions for the prefactors that involve contributions of higher than second order in $\gamma$-matrices.

\subsection{Cubic vertices for totally symmetric arbitrary spin fields}

We now consider cubic vertices for totally symmetric fields with the mass values given in (10.1) i.e., the massive bosonic field carries external line index $a=1$, the massive fermionic field carries external line index $a=2$, while the massless fermionic field corresponds to $a=3$. To study the totally symmetric fields we set $\nu=1$ in (10.2), (10.3) and ignore the contribution of the oscillators with $n>1$. To simplify the formulas we drop the oscillator's subscript $n=1$ and use the simplified notation for oscillators: $\alpha^{(a) I} \equiv \alpha_{1}^{(a) I}, \zeta^{(a)} \equiv \zeta_{1}^{(a)}$. In doing so, we obtain, using the solution given (10.2), (10.3), the cubic interaction vertices for the totally symmetric fields,

$$
\begin{array}{ll}
p_{[3]}^{-}=K^{(23)} V^{\mathrm{K}}\left(L^{(1)}, L^{(2)} ; Q^{(a a+1)}\right), & \text { K-vertex; } \\
p_{[3]}^{-}=F V^{\mathrm{F}}\left(L^{(1)}, L^{(2)} ; Q^{(a a+1)}\right), & \text { F-vertex; }
\end{array}
$$

where we use the notation

$$
\begin{aligned}
F \equiv & \frac{1}{\beta_{2} \beta_{3}} p_{\theta_{2}}\left(\frac{\check{\beta}_{1}}{\beta_{1}} \mathbb{P}^{I}-\gamma^{I J} \mathbb{P}^{J}+\beta_{3} \mathrm{~m}_{2} \gamma^{I} \gamma_{*}\right) \eta_{3} \alpha^{(1) I} \\
& +\frac{1}{\beta_{2} \beta_{3}} p_{\theta_{2}}\left(-\mathrm{m}_{2} \mathbb{P}^{I} \gamma^{I} \gamma_{*}+\beta_{3} \mathrm{~m}_{2}^{2}+\frac{2 \beta_{2} \beta_{3}}{\beta_{1}} \mathrm{~m}_{1}^{2}\right) \eta_{3} \frac{\zeta^{(1)}}{\mathrm{m}_{1}} \\
& L^{(1)} \equiv B^{(1)}-\frac{\mathrm{m}_{2}^{2}}{2 \mathrm{~m}_{1}} \zeta^{(1)}, \quad L^{(2)} \equiv B^{(2)}+\frac{\mathrm{m}_{1}^{2}}{2 \mathrm{~m}_{2}} \zeta^{(2)}
\end{aligned}
$$




$$
\begin{aligned}
& B^{(a)} \equiv \frac{\alpha^{(a) I} \mathbb{P}^{I}}{\beta_{a}}-\frac{\check{\beta}_{a}}{2 \beta_{a}} \mathrm{~m}_{a} \zeta^{(a)}, \quad a=1,2 ; \\
& B^{(3)} \equiv \frac{\alpha^{(3) I} \mathbb{P}^{I}}{\beta_{3}}, \\
& Q^{(12)} \equiv \alpha^{(12)}-\frac{\zeta^{(2)}}{\mathrm{m}_{2}} B^{(1)}+\frac{\zeta^{(1)}}{\mathrm{m}_{1}} B^{(2)}, \\
& Q^{(23)} \equiv \alpha^{(23)}-\frac{\mathrm{m}_{2} \zeta^{(2)}}{\mathrm{m}_{1}^{2}-\mathrm{m}_{2}^{2}} B^{(3)}-\frac{2}{\mathrm{~m}_{1}^{2}-\mathrm{m}_{2}^{2}} B^{(2)} B^{(3)} \\
& Q^{(31)} \equiv \alpha^{(31)}-\frac{\mathrm{m}_{1} \zeta^{(1)}}{\mathrm{m}_{1}^{2}-\mathrm{m}_{2}^{2}} B^{(3)}+\frac{2}{\mathrm{~m}_{1}^{2}-\mathrm{m}_{2}^{2}} B^{(3)} B^{(1)} .
\end{aligned}
$$

The quadratic forms $\alpha^{(a b)}$ and the prefactor $K^{(23)}$ are defined in (4.17) and (10.4) respectively. Solution given in (10.12), (10.13) provides the complete list of parity invariant cubic interaction for totally symmetric field with mass parameters given in (10.1). We now turn to vertices for the totally symmetric fields with fixed spin values.

\subsubsection{Cubic vertices for totally symmetric fields with fixed but arbitrary spin values}

Discussion of cubic vertices for one massless fermionic field, one massive fermionic field and one massive bosonic field with different mass values is similar to the one in Section 8.1.1. This, we consider vertices involving one massive spin- $s^{(1)}$ bosonic field, one massive spin- $\left(s^{(2)}+\frac{1}{2}\right)$ fermionic field and one massless spin- $\left(s^{(3)}+\frac{1}{2}\right)$ fermionic field, where the massive fermionic and massive bosonic fields have different values of the mass parameter:

$$
\begin{array}{lll}
\mathrm{m}_{1} \neq 0, & \mathrm{~m}_{2} \neq 0, \quad \mathrm{~m}_{1} \neq \mathrm{m}_{2}, & \mathrm{~m}_{3}=0, \\
s^{(1)}, & s^{(2)}+\frac{1}{2}, & s^{(3)}+\frac{1}{2} .
\end{array}
$$

The cubic vertices for fields with the spin and mass values as in (10.21) can be obtained by solving Eqs. 8.21), (8.22) with $p_{[3]}^{-}$given in (10.12), (10.13). We then obtain the cubic vertices

$$
\begin{array}{rlrl}
p_{[3]}^{-}\left(s^{(1)}, s^{(2)}+\frac{1}{2},\right. & \left.s^{(3)}+\frac{1}{2} ; w^{(1)}, w^{(2)}\right) & \\
& =K^{(23)}\left(L^{(1)}\right)^{w^{(1)}}\left(L^{(2)}\right)^{w^{(2)}} \prod_{a=1}^{3}\left(Q^{(a a+1)}\right)^{y^{(a+2)}}, & & \text { K-vertex; } \\
& =F\left(L^{(1)}\right)^{w^{(1)}}\left(L^{(2)}\right)^{w^{(2)}} \prod_{a=1}^{3}\left(Q^{(a a+1)}\right)^{y^{(a+2)}}, & \text { F-vertex; }
\end{array}
$$

where the parameters $y^{(a)}$ are given by

$$
\begin{array}{ll}
y^{(1)}=\frac{1}{2}\left(s^{(2)}+s^{(3)}-s^{(1)}+w^{(1)}-w^{(2)}\right), \\
y^{(2)}=\frac{1}{2}\left(s^{(1)}+s^{(3)}-s^{(2)}-w^{(1)}+w^{(2)}\right), \\
y^{(3)}=\frac{1}{2}\left(s^{(1)}+s^{(2)}-s^{(3)}-w^{(1)}-w^{(2)}\right), & \text { for K-vertex; }
\end{array}
$$




$$
\begin{aligned}
& y^{(1)}=\frac{1}{2}\left(s^{(2)}+s^{(3)}-s^{(1)}+w^{(1)}-w^{(2)}+1\right), \\
& y^{(2)}=\frac{1}{2}\left(s^{(1)}+s^{(3)}-s^{(2)}-w^{(1)}+w^{(2)}-1\right), \\
& y^{(3)}=\frac{1}{2}\left(s^{(1)}+s^{(2)}-s^{(3)}-w^{(1)}-w^{(2)}-1\right), \quad \text { for F-vertex. }
\end{aligned}
$$

Two integers $w^{(1)}, w^{(2)}$ are the freedom of our solution. For fixed spin values $s^{(1)}, s^{(2)}, s^{(3)}$, these integers label all possible cubic interaction vertices that can be built for the fields under consideration. For vertices (10.22), (10.23) to be sensible we impose the restrictions

$$
\begin{array}{lll}
w^{(1)} \geq 0, \quad w^{(2)} \geq 0, \quad y^{(a)} \geq 0, & a=1,2,3 ; & \\
\mathbf{s}-w^{(1)}-w^{(2)} \text { even integer, } & \text { for K-vertex; } \\
w^{(1)} \geq 0, \quad w^{(2)} \geq 0, \quad y^{(a)} \geq 0, & a=1,2,3 ; & \\
\mathbf{s}-w^{(1)}-w^{(2)} \text { odd integer }, & \text { for F-vertex. }
\end{array}
$$

which amount to the requirement that the powers of all forms in (10.22), (10.23) be non-negative integers. The maximal and minimal number of powers of $\mathbb{P}^{I}$ in 10.22), 10.23), denoted by $k_{\max }$ and $k_{\min }$ respectively, are given by 17

$$
\begin{array}{lll}
k_{\max }=\frac{1}{2}\left(s^{(1)}+s^{(2)}+3 s^{(3)}+w^{(1)}+w^{(2)}\right)+1, & k_{\min }=0, & \text { for K-vertex; } \\
k_{\max }=\frac{1}{2}\left(s^{(1)}+s^{(2)}+3 s^{(3)}+w^{(1)}+w^{(2)}+1\right), & k_{\min }=0, & \text { for F-vertex. }
\end{array}
$$

We note that using (10.24),(10.25) allows rewriting restrictions (10.26), (10.27) in the equivalent form 18

$$
\begin{array}{ll}
\left|s^{(1)}-s^{(2)}-w^{(1)}+w^{(2)}\right| \leq s^{(3)} \leq s^{(1)}+s^{(2)}-w^{(1)}-w^{(2)}, & \text { for K-vertex; } \\
\left|s^{(1)}-s^{(2)}-w^{(1)}+w^{(2)}-1\right| \leq s^{(3)} \leq s^{(1)}+s^{(2)}-w^{(1)}-w^{(2)}-1, & \text { for F-vertex; }
\end{array}
$$

\section{Cubic vertices for two massive fermionic fields and one massive bosonic field}

We finally consider the cubic interaction vertex for three mixed-symmetry massive fields with mass parameters given by

$$
\left.\mathrm{m}_{1}\right|_{F} \neq 0,\left.\quad \mathrm{~m}_{2}\right|_{F} \neq 0,\left.\quad \mathrm{~m}_{3}\right|_{B} \neq 0 .
$$

The solution we found for $\mathrm{K}$ - and F-vertices is given by

$$
p_{[3]}^{-}=K^{(12)} V^{\mathrm{K}}\left(L_{n}^{(a)} ; Q_{m n}^{(a a+1)}\right), \quad \quad \text { K-vertex; }
$$

\footnotetext{
${ }^{17}$ Expressions for $K^{(12)}$ (10.4) and $F, L^{(a)}, Q^{(a a+1)}$ (10.14)-(10.20) imply that $k_{\max }=w^{(1)}+w^{(2)}+2 y^{(1)}+2 y^{(2)}+$ $y^{(3)}+1$ for K-vertex and $k_{\max }=w^{(1)}+w^{(2)}+2 y^{(1)}+2 y^{(2)}+y^{(3)}+1$ for F-vertex. Relations for $y^{(a)}$ (10.24), (10.25) then lead to $k_{\max }$ given in (10.28),(10.29).

${ }^{18}$ If $w^{(1)}=w^{(2)}=0$, then restriction (10.30) becomes the restrictions well known in the angular momentum theory, $\left|s^{(1)}-s^{(2)}\right| \leq s^{(3)} \leq s^{(1)}+s^{(2)}$, while restriction (10.31) takes the form $\left|s^{(1)}-s^{(2)}-1\right| \leq s^{(3)} \leq s^{(1)}+s^{(2)}-1$.
} 


$$
p_{[3]}^{-}=F_{n} V^{\mathrm{F}}\left(L_{n}^{(a)} ; Q_{m n}^{(a a+1)}\right), \quad \text { F-vertex; }
$$

where $V^{\mathrm{K}}, V^{\mathrm{F}}$ are arbitrary polynomials of linear and quadratic forms and we use the notation

$$
\begin{aligned}
& K^{(12)} \equiv \frac{1}{\beta_{1} \beta_{2}} p_{\theta_{1}}\left(\mathbb{P}^{I} \gamma^{I} \gamma_{*}-\mathrm{m}_{12}\right) \eta_{2} \\
& F_{n} \equiv \frac{1}{\beta_{1} \beta_{2}} p_{\theta_{1}}\left(\frac{\check{\beta}_{3}}{\beta_{3}} \mathbb{P}^{I}-\gamma^{I J} \mathbb{P}^{J}+\left(\mathrm{m}_{1} \beta_{2}+\mathrm{m}_{2} \beta_{1}\right) \gamma^{I} \gamma_{*}\right) \eta_{2} \zeta_{n}^{(3) I} \\
&+\frac{1}{\beta_{1} \beta_{2}} p_{\theta_{1}}\left(-\check{\mathrm{m}}_{3} \mathbb{P}^{I} \gamma^{I} \gamma_{*}+\check{\mathrm{m}}_{3} \mathrm{~m}_{12}+\frac{2 \beta_{1} \beta_{2}}{\beta_{3}} \mathrm{~m}_{3}^{2}\right) \eta_{2} \frac{\zeta_{n}^{(3)}}{\mathrm{m}_{3}} \\
& L_{n}^{(a)} \equiv B_{n}^{(a)}-\frac{\mathrm{m}_{a+1}^{2}-\mathrm{m}_{a+2}^{2}}{2 \mathrm{~m}_{a}} \zeta_{n}^{(a)} \\
& B_{n}^{(a)} \equiv \frac{\alpha_{n}^{(a) I} \mathbb{P}^{I}}{\beta_{a}}-\frac{\check{\beta}_{a}}{2 \beta_{a}} \mathrm{~m}_{a} \zeta_{n}^{(a)}, \\
& Q_{m n}^{(a a+1)} \equiv \alpha_{m n}^{(a a+1)}-\frac{\zeta_{n}^{(a+1)}}{\mathrm{m}_{a+1}} B_{m}^{(a)}+\frac{\zeta_{m}^{(a)}}{\mathrm{m}_{a}} B_{n}^{(a+1)}-\frac{\mathrm{m}_{a+2}^{2}}{2 \mathrm{~m}_{a} \mathrm{~m}_{a+1}} \zeta_{m}^{(a)} \zeta_{n}^{(a+1)} \\
& \mathrm{m}_{a b} \equiv \mathrm{m}_{a} \beta_{b}-\mathrm{m}_{b} \beta_{a}, \quad \check{\mathrm{m}}_{a} \equiv \mathrm{m}_{a+1}-\mathrm{m}_{a+2} \cdot
\end{aligned}
$$

The quadratic forms $\alpha_{m n}^{(a b)}$ are defined in (4.8). From (11.5),(11.6), (11.8), we see that the prefactors $F_{n}$, the linear forms $L_{n}^{(a)}$, and the quadratic forms $Q_{m n}^{(a a+1)}$ are singular in the massless limit, $\mathrm{m}_{a} \rightarrow 0$, $a=1,2,3$. This implies that, in general, the cubic vertices for massive fields are also singular as $\mathrm{m}_{a} \rightarrow 0, a=1,2,3$. Note that, in contrast to the prefactors $F_{n}$, the prefactor $K^{(12)}$ has smooth massless limit. Solution for $V^{\mathrm{K}}, V^{\mathrm{F}}$ in (11.2), (11.3) is complete solution, while the solution for the prefactors $K^{(12)}, F_{n}$ in (11.4), (11.5) is not complete solution. Namely, for the vertices of mixedsymmetry fields there are extra solutions for the prefactors that involve contribution of higher than second order in $\gamma$-matrices.

\subsection{Cubic vertices for totally symmetric two massive fermionic fields and one massive bosonic field}

We now consider cubic vertices for totally symmetric fields with the mass values given in (11.1), i.e., the massive fermionic fields carry external line indices $a=1,2$, while the massive bosonic field carries external line index $a=3$. Because for the studying the totally symmetric fields it is sufficient to use one sort of oscillators, we set $\nu=1$ in (11.2), (11.3) and ignore the contribution of the oscillators with $n>1$. To simplify the formulas we drop the oscillator's subscript $n=1$ and use the simplified notation for oscillators: $\alpha^{(a) I} \equiv \alpha_{1}^{(a) I}, \zeta^{(a)} \equiv \zeta_{1}^{(a)}$. In doing so, we obtain, using the solution given in (11.2) (11.3), the cubic interaction vertices for totally symmetric fields,

$$
\begin{aligned}
& p_{[3]}^{-}=K^{(12)} V^{\mathrm{K}}\left(L^{(a)} ; Q^{(a a+1)}\right), \quad \text { K-vertex; } \\
& p_{[3]}^{-}=F V^{\mathrm{F}}\left(L^{(a)} ; Q^{(a a+1)}\right), \quad \quad \text { F-vertex; }
\end{aligned}
$$

where we use the notation

$$
F \equiv \frac{1}{\beta_{1} \beta_{2}} p_{\theta_{1}}\left(\frac{\check{\beta}_{3}}{\beta_{3}} \mathbb{P}^{I}-\gamma^{I J} \mathbb{P}^{J}+\left(\mathrm{m}_{1} \beta_{2}+\mathrm{m}_{2} \beta_{1}\right) \gamma^{I} \gamma_{*}\right) \eta_{2} \alpha^{(3) I}
$$




$$
\begin{aligned}
& +\frac{1}{\beta_{1} \beta_{2}} p_{\theta_{1}}\left(-\check{\mathrm{m}}_{3} \mathbb{P}^{I} \gamma^{I} \gamma_{*}+\check{\mathrm{m}}_{3} \mathrm{~m}_{12}+\frac{2 \beta_{1} \beta_{2}}{\beta_{3}} \mathrm{~m}_{3}^{2}\right) \eta_{2} \frac{\zeta^{(3)}}{\mathrm{m}_{3}} \\
& L^{(a)} \equiv B^{(a)}-\frac{\mathrm{m}_{a+1}^{2}-\mathrm{m}_{a+2}^{2}}{2 \mathrm{~m}_{a}} \zeta^{(a)}, \\
& B^{(a)} \equiv \frac{\alpha^{(a) I} \mathbb{P}^{I}}{\beta_{a}}-\frac{\check{\beta}_{a}}{2 \beta_{a}} \mathrm{~m}_{a} \zeta^{(a)}, \\
& Q^{(a a+1)} \equiv \alpha^{(a a+1)}-\frac{\zeta^{(a+1)}}{\mathrm{m}_{a+1}} B^{(a)}+\frac{\zeta^{(a)}}{\mathrm{m}_{a}} B^{(a+1)}-\frac{\mathrm{m}_{a+2}^{2}}{2 \mathrm{~m}_{a} \mathrm{~m}_{a+1}} \zeta^{(a)} \zeta^{(a+1)} \\
& \mathrm{m}_{a b} \equiv \mathrm{m}_{a} \beta_{b}-\mathrm{m}_{b} \beta_{a}, \quad \check{\mathrm{m}}_{a} \equiv \mathrm{m}_{a+1}-\mathrm{m}_{a+2},
\end{aligned}
$$

while $\alpha^{(a b)}$ and $K^{(12)}$ are defined in (4.17) and (11.4) respectively. We note that solution for vertices given in (11.10), (11.11) is the complete solution. In other words, vertices in (11.10), (11.11) constitute the complete list of parity invariant cubic vertices for massive totally symmetric fields. We now restrict our attention to vertices for totally symmetric fields with fixed spin values.

\subsubsection{Cubic vertices for totally symmetric fields with fixed but arbitrary spin values}

Vertices (11.10), (11.11) describe interaction of the towers of massive totally symmetric fields (2.16), (2.30). We next obtain vertices for massive spin $s^{(1)}+\frac{1}{2}, s^{(2)}+\frac{1}{2}$ and $s^{(3)}$ fields. Namely, we consider vertices involving two spin $s^{(1)}+\frac{1}{2}$ and $s^{(2)}+\frac{1}{2}$ fermionic fields having the respective mass parameters $m_{1}$ and $m_{2}$ and one spin- $s^{(3)}$ bosonic field having the mass parameter $m_{3}$ :

$$
\begin{array}{lll}
\left.\mathrm{m}_{1}\right|_{F} \neq 0, & \left.\mathrm{~m}_{2}\right|_{F} \neq 0, & \left.\mathrm{~m}_{3}\right|_{B} \neq 0, \\
s^{(1)}+\frac{1}{2}, & s^{(2)}+\frac{1}{2}, & s^{(3)} .
\end{array}
$$

The massive spin- $\left(s^{(1)}+\frac{1}{2}\right)$ and $-\left(s^{(2)}+\frac{1}{2}\right)$ fermionic fields are described by the respective ketvectors $\left|\psi_{s^{(1)}}\right\rangle,\left|\psi_{s^{(2)}}\right\rangle$, while the massive spin- $s^{(3)}$ bosonic field is described by ket-vector $\left|\phi_{s^{(3)}}\right\rangle$. The ket-vectors of massive fields $\left|\psi_{s(a)}\right\rangle, a=1,2$ and $\left|\phi_{s(3)}\right\rangle$, can be obtained from the respective expressions in (2.23) and (2.11) by replacing $s \rightarrow s^{(a)}, \alpha^{I} \rightarrow \alpha^{(a) I}, \zeta \rightarrow \zeta^{(a)}$. Because $\left|\psi_{s^{(1)}}\right\rangle$, $\left|\psi_{s}(2)\right\rangle,\left|\phi_{s^{(3)}}\right\rangle$ are respective degree- ${ }^{(a)}$ homogeneous polynomials in $\alpha^{(a) I}, \zeta^{(a)}$, it is obvious that the vertices we are interested in must satisfy the equations

$$
\left(\alpha^{(a) I} \bar{\alpha}^{(a) I}+\zeta^{(a)} \bar{\zeta}^{(a)}-s^{(a)}\right)\left|p_{[3]}^{-}\right\rangle=0, \quad a=1,2,3,
$$

which tell us that the vertices must be degree- $s^{(a)}$ homogeneous polynomials in the oscillators $\alpha^{(a) I}, \zeta^{(a)}$. Taking into account that the prefactor $F$ and the linear forms $L^{(a)}$ are degree-1 homogeneous polynomials in oscillators, while the quadratic forms $Q^{(a a+1)}$ are degree-2 homogeneous polynomials in the oscillators we obtain the general solution of Eqs.(11.18) as

$$
\begin{array}{ll}
p_{[3]}^{-}\left(s^{(1)}+\frac{1}{2}, s^{(2)}+\frac{1}{2}, s^{(3)} ; w^{(1)}, w^{(2)}, w^{(3)}\right)=K^{(12)} \prod_{a=1}^{3}\left(L^{(a)}\right)^{w^{(a)}}\left(Q^{(a a+1)}\right)^{y^{(a+2)}}, \quad \text { K-vertex; } \\
p_{[3]}^{-}\left(s^{(1)}+\frac{1}{2}, s^{(2)}+\frac{1}{2}, s^{(3)} ; w^{(1)}, w^{(2)}, w^{(3)}\right)=F \prod_{a=1}^{3}\left(L^{(a)}\right)^{w^{(a)}}\left(Q^{(a a+1)}\right)^{y^{(a+2)}}, & \text { F-vertex; }
\end{array}
$$


where integers $y^{(a)}$ are expressible in terms of $s^{(a)}$ and three integers $w^{(a)}$ labeling the freedom of our solution,

$$
\begin{aligned}
& y^{(a)}=\frac{1}{2}\left(\mathbf{s}+w^{(a)}-w^{(a+1)}-w^{(a+2)}\right)-s^{(a)}, \quad a=1,2,3, \quad \text { for K-vertex; } \\
& y^{(a)}=\frac{1}{2}\left(\mathbf{s}+w^{(a)}-w^{(a+1)}-w^{(a+2)}-1\right)-s^{(a)}, \quad a=1,2 ; \\
& y^{(3)}=\frac{1}{2}\left(\mathbf{s}+w^{(3)}-w^{(1)}-w^{(2)}+1\right)-s^{(3)}, \quad \text { for F-vertex; }
\end{aligned}
$$

and $s$ is given in (4.22). The maximal and minimal numbers of powers of $\mathbb{P}^{I}$ in (11.19), (11.20), denoted by $k_{\max }$ and $k_{\min }$ respectively, are given by 19

$$
\begin{array}{lll}
k_{\max }=\frac{1}{2}\left(\mathbf{s}+\sum_{a=1}^{3} w^{(a)}\right)+1, & k_{\min }=0, & \text { for K-vertex; } \\
k_{\max }=\frac{1}{2}\left(\mathbf{s}+\sum_{a=1}^{3} w^{(a)}+1\right), & k_{\min }=0, & \text { for F-vertex. }
\end{array}
$$

Requiring the powers of the forms $L^{(a)}$ and $Q^{(a a+1)}$ in (11.19), (11.20) to be non-negative integers gives the restrictions

$$
\begin{array}{lll}
w^{(a)} \geq 0, & y^{(a)} \geq 0, \quad a=1,2,3 ; & \\
\mathbf{s}+\sum_{a=1}^{3} w^{(a)} & \text { even integer, } & \text { for K-vertex; } \\
w^{(a)} \geq 0, \quad y^{(a)} \geq 0, \quad a=1,2,3 ; & \\
\mathbf{s}+\sum_{a=1}^{3} w^{(a)} & \text { odd integer }, & \text { for F-vertex. }
\end{array}
$$

Using relations (11.21), (11.22) allows rewriting restrictions (11.25),(11.26) as

$$
\begin{aligned}
& s^{(3)}-s^{(1)}-s^{(2)}+w^{(1)}+w^{(2)} \leq w^{(3)} \leq s^{(3)}-\left|s^{(1)}-s^{(2)}-w^{(1)}+w^{(2)}\right|, \quad \text { for K-vertex; } \\
& s^{(3)}-s^{(1)}-s^{(2)}+w^{(1)}+w^{(2)}-1 \leq w^{(3)} \leq s^{(3)}-\left|s^{(1)}-s^{(2)}-w^{(1)}+w^{(2)}\right|-1,
\end{aligned}
$$

for F-vertex.

Examples of light-cone cubic vertices and their counterparts in manifestly Lorentz invariant approach. We now give examples of vertices for particular spin values and make comment concerning hermitian properties of our vertices.

Cubic vertices for two massive spin- $\frac{1}{2}$ and spin- $\frac{1}{2}$ fermionic fields having the respective mass parameters $\mathrm{m}_{1}, \mathrm{~m}_{2}$ and one massive spin- $s$ bosonic field with the mass parameter $\mathrm{m}_{3}$ are given by

$$
p_{[3]}^{-}\left(\frac{1}{2}, \frac{1}{2}, s ; 0,0, s\right)=K^{(12)}\left(L^{(3)}\right)^{s},
$$

\footnotetext{
${ }^{19}$ Expressions for $K^{(12)}$ 11.4 and $F, L^{(a)}, Q^{(a a+1)}$ 11.12)-11.15 imply that $k_{\max }=1+\sum_{a=1}^{3}\left(w^{(a)}+y^{(a)}\right)$. Taking $y^{(a)}$ 11.21, (11.22) into account we then find $k_{\max }$ given in (11.23), 11.24).

${ }^{20}$ If $w^{(a)}=0, a=1,2,3$, then restrictions 11.27 becomes the restrictions well known in the angular momentum theory: $\left|s^{(1)}-s^{(2)}\right| \leq s^{(3)} \leq s^{(1)}+s^{(2)}$, while restriction 11.28) takes the form $\left|s^{(1)}-s^{(2)}\right| \leq s^{(3)}-1 \leq s^{(1)}+s^{(2)}$.
} 


$$
p_{[3]}^{-}\left(\frac{1}{2}, \frac{1}{2}, s ; 0,0, s-1\right)=F\left(L^{(3)}\right)^{s-1} .
$$

Consider vertices (11.29), (11.30) for $s=1$. Manifestly Lorentz invariant K-vertex corresponding to our light-cone K-vertex (11.29) with $s=1$ takes the form

$$
\mathcal{L}=\bar{\psi}_{1} \gamma^{A B} \psi_{2} F^{A B}, \quad F^{A B} \equiv \partial^{A} \phi^{B}-\partial^{B} \phi^{A}
$$

while the manifestly Lorentz invariant F-vertex corresponding to our light-cone F-vertex (11.30) with $s=1$ is given by

$$
\mathcal{L}=\bar{\psi}_{1} \gamma^{A} \psi_{2}\left(\phi^{A}+\frac{1}{\mathrm{~m}_{3}} \partial^{A} \phi\right)
$$

In (11.32), $\phi^{A}$ and $\phi$ are vector and scalar fields of the Lorentz algebra which are used in gauge invariant Stueckelberg formulation of spin-1 massive field having mass parameter $m_{3}$. We recall that the scalar field $\phi$ is the Stueckelberg field.

Light-cone gauge vertex (11.29) and manifestly Lorentz invariant vertex (11.31) are not hermitian in general. We can obtain hermitian light-cone gauge and covariant vertices in the standard way: by adding or subtracting appropriate hermitian conjugated expression. In this way, we obtain the two well-known covariant vertices

$$
\begin{aligned}
\mathcal{L} & =\left(\bar{\psi}_{1} \gamma^{A B} \psi_{2}+\bar{\psi}_{2} \gamma^{A B} \psi_{1}\right) F^{A B} \\
\mathrm{i} \mathcal{L} & =\left(\bar{\psi}_{1} \gamma^{A B} \psi_{2}-\bar{\psi}_{2} \gamma^{A B} \psi_{1}\right) F^{A B} .
\end{aligned}
$$

In the same way we can consider vertices in (11.30) and (11.32). Namely, light-cone gauge vertex (11.30) and covariant vertex (11.32) are not hermitian when $\mathrm{m}_{1} \neq \mathrm{m}_{2}$, i.e., when $\psi_{1} \neq$ $\psi_{2}$. The hermitian light-cone gauge and covariant vertices are obtained by adding or subtracting appropriate hermitian conjugated expression,

$$
\begin{aligned}
\mathcal{L} & =\left(\bar{\psi}_{1} \gamma^{A} \psi_{2}+\bar{\psi}_{2} \gamma^{A} \psi_{1}\right)\left(\phi^{A}+\frac{1}{\mathrm{~m}_{3}} \partial^{A} \phi\right), \\
\mathrm{i} \mathcal{L} & =\left(\bar{\psi}_{1} \gamma^{A} \psi_{2}-\bar{\psi}_{2} \gamma^{A} \psi_{1}\right)\left(\phi^{A}+\frac{1}{\mathrm{~m}_{3}} \partial^{A} \phi\right) .
\end{aligned}
$$

To summarize, in order to obtain hermitian vertices we should supplement our vertices by appropriate hermitian conjugated expressions. Note also that, for the space-time dimensions when Dirac fields allow restriction to Majorana fields, our vertices are automatically hermitian.

Vertices for massive spin- $\frac{3}{2}$ fermionic field and massive spin- $s$ bosonic field. For the reader convenience we worked out the explicit expressions for cubic K- and F-vertices, (11.19), (11.20) involving two massive spin- $\frac{3}{2}$ fermionic fields and one massive spin- $s$ bosonic field,

$$
\begin{aligned}
& p_{[3]}^{-}\left(\frac{3}{2}, \frac{3}{2}, s ; 0,0, s-2\right)=K^{(12)}\left(L^{(3)}\right)^{s-2} Q^{(23)} Q^{(31)} \\
& p_{[3]}^{-}\left(\frac{3}{2}, \frac{3}{2}, s ; 0,0, s\right)=K^{(12)}\left(L^{(3)}\right)^{s} Q^{(12)} \\
& p_{[3]}^{-}\left(\frac{3}{2}, \frac{3}{2}, s ; 0,1, s-1\right)=K^{(12)} L^{(2)}\left(L^{(3)}\right)^{s-1} Q^{(31)} \\
& p_{[3]}^{-}\left(\frac{3}{2}, \frac{3}{2}, s ; 1,1, s\right)=K^{(12)} L^{(1)} L^{(2)}\left(L^{(3)}\right)^{s}
\end{aligned}
$$




$$
\begin{aligned}
& p_{[3]}^{-}\left(\frac{3}{2}, \frac{3}{2}, s ; 0,0, s-3\right)=F\left(L^{(3)}\right)^{s-3} Q^{(23)} Q^{(31)}, \\
& p_{[3]}^{-}\left(\frac{3}{2}, \frac{3}{2}, s ; 0,0, s-1\right)=F\left(L^{(3)}\right)^{s-1} Q^{(12)}, \\
& p_{[3]}^{-}\left(\frac{3}{2}, \frac{3}{2}, s ; 0,1, s-2\right)=F L^{(2)}\left(L^{(3)}\right)^{s-2} Q^{(31)} \\
& p_{[3]}^{-}\left(\frac{3}{2}, \frac{3}{2}, s ; 1,1, s-1\right)=F L^{(1)} L^{(2)}\left(L^{(3)}\right)^{s-1} .
\end{aligned}
$$

Acknowledgments. This work was supported by the INTAS project 03-51-6346, by the RFBR Grant No.05-02-17654, RFBR Grant for Leading Scientific Schools, Grant No. LSS-4401.2006.2 and the Alexander von Humboldt Foundation Grant PHYS0167.

\section{Appendix A Notation}

Notation in basis of Lorentz algebra $s o(d-1,1)$. Our conventions are as follows. $x^{A}$ denotes coordinates in $d$-dimensional flat space-time, while $\partial_{A}$ denotes derivatives with respect to $x^{A}$, $\partial_{A} \equiv \partial / \partial x^{A}$. Vector indices of the Lorentz algebra $s o(d-1,1)$ take the values $A, B, C, E=$ $0,1, \ldots, d-1$. We use the mostly positive flat metric tensor $\eta^{A B}$. For simplicity, we drop $\eta_{A B}$ in the scalar products, i.e., we use $X^{A} Y^{A} \equiv \eta_{A B} X^{A} Y^{B}$.

$\gamma^{A}$ and $\Gamma_{*}$-matrices for even $d$. For $d=2 \mathrm{n}, \gamma^{A}$-matrices are $2^{\mathrm{n}} \times 2^{\mathrm{n}}$ Dirac matrices in $d$ dimensional Minkowski space, $\left\{\gamma^{A}, \gamma^{B}\right\}=2 \eta^{A B}$, while the matrix $\Gamma_{*}$ is defined as

$$
\Gamma_{*} \equiv \epsilon \gamma^{0} \gamma^{1} \ldots \gamma^{d-1}, \quad \Gamma_{*}^{2}=1
$$

$\gamma^{A}$ and $\Gamma_{*}$-matrices for odd $d$. For $d=2 \mathrm{n}+1$, we use matrices $\gamma^{A}, \Gamma_{*}$ which are the $2^{\mathrm{n}+1} \times 2^{\mathrm{n}+1}$ Dirac matrices in $d+1$ dimensional Minkowski space,

$$
\left\{\gamma^{A}, \gamma^{B}\right\}=2 \eta^{A B}, \quad\left\{\gamma^{A}, \Gamma_{*}\right\}=0, \quad \Gamma_{*}^{2}=1
$$

The use of such matrices implies that Lagrangian for $2^{\mathrm{n}+1}$ th component Dirac field $\Psi$ given by

$$
{ }_{1} \mathcal{L}=\bar{\Psi}\left(\gamma^{A} \partial^{A}+\mathrm{m}\right) \Psi
$$

describes two massive spin- $\frac{1}{2}$ Dirac fields, when $d$ is odd. In order to obtain description of massive spin- $\frac{1}{2}$ field in terms of $2^{n}$ th component Dirac fields we introduce the decomposition

$$
\begin{aligned}
& \Psi=\Psi_{+}+\Psi_{-}, \quad \Psi_{ \pm} \equiv \frac{1}{2}\left(1 \pm \sigma_{*}\right) \Psi, \\
& \sigma_{*} \equiv 1^{\mathrm{n}+1} \gamma^{0} \ldots \gamma^{d-1}, \quad\left[\gamma^{A}, \sigma_{*}\right]=0, \quad \sigma_{*}^{2}=1, \quad \sigma_{*}^{\dagger}=\sigma_{*} .
\end{aligned}
$$

In terms of $2^{\mathrm{n}}$ th component Dirac fields $\Psi_{ \pm}$, Lagrangian (A.3) takes the form

$$
{ }_{1} \mathcal{L}=\bar{\Psi}_{+}\left(\gamma^{A} \partial^{A}+\mathrm{m}\right) \Psi_{+}+\bar{\Psi}_{-}\left(\gamma^{A} \partial^{A}+\mathrm{m}\right) \Psi_{-} .
$$

Thus, for odd $d$, our description of massive fields involves reducible set of massive fields. We use such description in order to treat the fermionic fields in odd and even dimensions on an equal 
footing. For the case of odd $d$, the description for single massive field can be obtained by using decomposition given in (A.4). We note also that, for odd $d$, use of the matrix $\Gamma_{*}$ is motivated by the desire to get constraints for the fermionic field in the form given in (2.21) (for details, see below).

We use matrices constructed out of $\gamma^{A}$ and $\Gamma_{*}$ matrices,

$$
\gamma^{A B} \equiv \frac{1}{2} \gamma^{A} \gamma^{B}-(A \leftrightarrow B), \quad U \equiv \frac{1}{\sqrt{2}}\left(1+\mathrm{i} \Gamma_{*}\right)
$$

The $\gamma^{A}$-matrices satisfy the hermitian conjugation rule given by $\gamma^{A \dagger}=\gamma^{0} \gamma^{A} \gamma^{0}$.

Notation in basis of $s o(d-2)$ algebra. We decompose the Lorentz frame space-time coordinates $x^{A}$ into light-cone frame space-time coordinates $x^{ \pm}, x^{I}$ defined by

$$
x^{ \pm} \equiv \frac{1}{\sqrt{2}}\left(x^{d-1} \pm x^{0}\right), \quad x^{I}, \quad I=1, \ldots, d-2,
$$

and treat $x^{+}$as an evolution parameter. Vector indices of the $s o(d-2)$ algebra take values $I, J, K=1, \ldots, d-2$. In the light-cone frame, vector of the Lorentz algebra $X^{A}$ is decomposed as $X^{+}, X^{-}, X^{I}$ and a scalar product of two vectors is then represented as

$$
\eta_{A B} X^{A} Y^{B}=X^{+} Y^{-}+X^{-} Y^{+}+X^{I} Y^{I}
$$

where the covariant and contravariant components of vectors are related as $X^{+}=X_{-}, X^{-}=X_{+}$, $X^{I}=X_{I}$. This leads to the following conventions for the derivatives:

$$
\partial^{I}=\partial_{I} \equiv \partial / \partial x^{I}, \quad \partial^{ \pm}=\partial_{\mp} \equiv \partial / \partial x^{\mp} .
$$

In the light-cone frame, we introduce the matrix $\gamma_{*}$ and projectors $\Pi^{\oplus}, \Pi^{\ominus}$ defined by

$$
\begin{array}{lll}
\Gamma_{*} \equiv \gamma^{+-} \gamma_{*}, & \Pi^{\oplus} \equiv \frac{1}{2} \gamma^{-} \gamma^{+}, & \Pi^{\ominus} \equiv \frac{1}{2} \gamma^{+} \gamma^{-}, \\
\Pi^{\oplus} \Pi^{\oplus}=\Pi^{\oplus}, & \Pi^{\ominus} \Pi^{\ominus}=\Pi^{\ominus}, & \Pi^{\oplus} \Pi^{\ominus}=0,
\end{array}
$$

Bosonic oscillators. We use a set of the bosonic creation operators $\alpha_{n}^{I}, \zeta_{n}$, and the respective set of annihilation operators $\bar{\alpha}_{n}^{I}, \bar{\zeta}_{n}, m, n=1, \ldots \nu$, where $\nu$ is arbitrary integer $\nu \geq 1$. These operators are referred to as oscillators. Commutation relations, the vacuum, and hermitian conjugation rules are defined as

$$
\begin{array}{ll}
{\left[\bar{\alpha}_{m}^{I}, \alpha_{n}^{J}\right]=\eta^{I J} \delta_{m n},} & {\left[\bar{\zeta}_{m}, \zeta_{n}\right]=\delta_{m n}, \quad \bar{\alpha}_{m}^{I}|0\rangle=0, \quad \bar{\zeta}_{m}|0\rangle=0,} \\
\alpha_{n}^{I \dagger}=\bar{\alpha}_{n}^{I}, & \zeta_{n}^{\dagger}=\bar{\zeta}_{n}, \quad m, n=1, \ldots \nu .
\end{array}
$$

To discuss totally symmetric field, it is sufficient to use one sort of oscillators $\alpha^{I}, \zeta, \bar{\alpha}^{I}, \bar{\zeta}$. Commutation relations, the vacuum, and hermitian conjugation rules are defined as

$$
\left[\bar{\alpha}^{I}, \alpha^{J}\right]=\eta^{I J}, \quad[\bar{\zeta}, \zeta]=1, \quad \bar{\alpha}^{I}|0\rangle=0, \quad \bar{\zeta}|0\rangle=0, \quad \alpha^{I \dagger}=\bar{\alpha}^{I}, \quad \zeta^{\dagger}=\bar{\zeta}
$$

Fermionic oscillators. We introduce fermionic oscillators $\theta^{\mathrm{a}}, \eta^{\mathrm{a}}, p_{\theta \mathrm{a}}, p_{\eta \mathrm{a}}$ subject to the algebraic constraints

$$
\Pi^{\oplus} \theta=\theta, \quad \Pi^{\oplus} \eta=\eta, \quad p_{\theta} \Pi^{\oplus}=p_{\theta}, \quad p_{\eta} \Pi^{\oplus}=p_{\eta},
$$


where the projector $\Pi^{\oplus}$ is defined in A.11). Anticommutation relations of the fermionic oscillators, the vacuum, and hermitian conjugation rules are defined as follows,

$$
\left\{\theta^{\mathrm{a}}, p_{\theta \mathrm{b}}\right\}=\Pi_{\mathrm{b}}^{\oplus \mathrm{a}}, \quad\left\{\eta^{\mathrm{a}}, p_{\eta \mathrm{b}}\right\}=\Pi_{\mathrm{b}}^{\oplus \mathrm{a}}, \quad \theta|0\rangle=0, \quad p_{\eta}|0\rangle=0, \quad \theta^{\dagger}=p_{\theta}, \quad \eta^{\dagger}=p_{\eta},
$$

where, in (A.16), the spinor indices a, b are shown explicitly.

Fourier transform of fields. Throughout this paper we use ket-vectors depending on the lightcone time $x^{+}$and momenta $p=\left\{\beta, p^{I}\right\}$. For the reader convenience we now explain how our ket-vectors are related to the one depending on space-time coordinates $x=\left\{x^{+}, x^{-}, x^{I}\right\}$. This is to say that for bosonic ket-vector $|\phi\rangle$ (2.9) and fermionic ket-vector $|\psi\rangle$ (2.22) we assume the following Fourier transform

$$
\begin{aligned}
|\phi(x, \alpha)\rangle= & \int \frac{d^{d-1} p}{(2 \pi)^{(d-1) / 2}} e^{\mathrm{i}\left(x^{+} p^{-}+x^{-} \beta+x^{I} p^{I}\right)} \mathrm{i}^{-N_{\zeta}}\left|\phi\left(x^{+}, p, \alpha\right)\right\rangle, \\
|\psi(x, \alpha)\rangle= & \int \frac{d^{d-1} p}{(2 \pi)^{(d-1) / 2}} e^{\mathrm{i}\left(x^{+} p^{-}+x^{-} \beta+x^{I} p^{I}\right)} \mathrm{i}^{-N_{\zeta}} \widehat{U}|\psi(p, \alpha)\rangle, \\
& \widehat{U} \equiv p_{\theta} U \theta+\left(U^{\dagger}\right)^{\mathrm{a}}{ }_{\mathrm{b}} \eta^{\mathrm{b}} \cdot p_{\eta \mathrm{a}}, \quad N_{\zeta} \equiv \sum_{n=1}^{\nu} \zeta_{n} \bar{\zeta}_{n},
\end{aligned}
$$

where $p^{-}$is given in (2.35), while the matrix $U$ is defined in (A.7). We assume that the ket-vector $|\psi(x, \alpha)\rangle$ is decomposed into the fermionic oscillators as in (2.17),

$$
|\psi(x, \alpha)\rangle=\left(p_{\theta} \psi(x, \alpha)+\psi^{\dagger}(x, \alpha) \eta\right)|0\rangle,
$$

while fields $\psi(x, \alpha), \psi^{\dagger}(x, \alpha)$ are decomposed into the bosonic oscillators as in (2.18), (2.19). The component fields entering $\psi(x, \alpha), \psi^{\dagger}(x, \alpha)$ are hermitian conjugated,

$$
\psi^{I_{1} \ldots I_{n}}(x)=\left(\psi^{\dagger I_{1} \ldots I_{n}}(x)\right)^{\dagger} .
$$

In terms of the Fourier modes, relation (A.21) implies

$$
\begin{array}{ll}
\psi_{s_{1} \ldots s_{\nu}}^{I_{1}^{1} \ldots I_{s_{1}}^{1} \ldots I_{1}^{\nu} \ldots I_{s_{\nu}}^{\nu}}(-p)=\left(\psi_{s_{1} \ldots s_{\nu}}^{\dagger I_{I_{1}}^{1} \ldots I_{1}^{1} \ldots I_{1}^{\nu} \ldots I_{s_{\nu}}^{\nu}}(p)\right)^{\dagger}, & \text { for massless field }, \\
\psi_{s_{1} \ldots s_{\nu}}^{I_{1}^{1} \ldots I_{t_{1}}^{1} \ldots I_{1}^{\nu} \ldots I_{t_{\nu}}^{\nu}}(-p)=(-)^{\sum_{n=1}^{\nu} t_{n}}\left(\psi_{s_{1} \ldots s_{\nu}}^{\dagger I_{1}^{1} \ldots I_{t_{1}}^{1} \ldots I_{1}^{\nu} \ldots I_{t_{\nu}}^{\nu}}(p)\right)^{\dagger}, & \text { for massive field } .
\end{array}
$$

We now make comment on the Fourier transform in (A.18). Using representation for ket-vector $|\psi(x, \alpha)\rangle$ in (A.20), we note that the ket-vector $\psi(x, \alpha)|0\rangle$ satisfies the standard constraint

$$
\left(\gamma^{I} \bar{\alpha}_{n}^{I}-\bar{\zeta}_{n}\right) \psi(x, \alpha)|0\rangle=0
$$

commonly used in the literature. It is the desire to get constraints in the form given in (2.21) that motivates us to use the Fourier transform given in (A.18).

Note also that fermionic fields in (2.18), (2.19) satisfy the algebraic constraint

$$
\Pi^{\oplus} \psi_{s_{1} \ldots s_{\nu}}(p, \alpha)=\psi_{s_{1} \ldots s_{\nu}}(p, \alpha)
$$




\section{References}

[1] P. A. M. Dirac, Rev. Mod. Phys. 21, 392 (1949).

[2] S. Weinberg, Phys. Rev. 150, 1313 (1966).

J. B. Kogut and D. E. Soper, Phys. Rev. D 1, 2901 (1970).

P. Goddard, J. Goldstone, C. Rebbi and C. B. Thorn, Nucl. Phys. B 56, 109 (1973).

[3] M. Kaku and K. Kikkawa, Phys. Rev. D 10, 1110 (1974).

J. F. L. Hopkinson, R. W. Tucker and P. A. Collins, Phys. Rev. D 12, 1653 (1975).

M. B. Green and J. H. Schwarz, Nucl. Phys. B 218, 43 (1983); Nucl. Phys. B 243, 475 (1984).

[4] M. B. Green, J. H. Schwarz and L. Brink, Nucl. Phys. B 219, 437 (1983).

[5] L. Brink, O. Lindgren and B. E. W. Nilsson, Nucl. Phys. B 212, 401 (1983).

S. Mandelstam, Nucl. Phys. B 213, 149 (1983).

L. Brink and A. Tollsten, Nucl. Phys. B 249, 244 (1985).

[6] M. B. Green and J. H. Schwarz, Phys. Lett. B 122, 143 (1983).

L. Brink, M. B. Green and J. H. Schwarz, Nucl. Phys. B 223, 125 (1983).

[7] R. R. Metsaev, Phys. Rev. D 71, 085017 (2005) [arXiv:hep-th/0410239].

[8] S. Ananth, L. Brink and P. Ramond, JHEP 0505, 003 (2005) [arXiv:hep-th/0501079].

[9] H.Hata et al., Phys.Rev.D34, 2360 (1986); Phys.Rev.D35, 1318 (1987).

W. Siegel and B. Zwiebach, Nucl. Phys. B 282, 125 (1987); Phys. Lett. B 184, 325 (1987).

W. Siegel, "Introduction To String Field Theory," arXiv:hep-th/0107094.

[10] A. K. H. Bengtsson, I. Bengtsson and L. Brink, Nucl. Phys. B 227, 31 (1983).

A. K. H. Bengtsson, I. Bengtsson and L. Brink, Nucl. Phys. B 227, 41 (1983).

A. K. H. Bengtsson, I. Bengtsson and N. Linden, Class. Quant. Grav. 4, 1333 (1987).

[11] E. S. Fradkin and R. R. Metsaev, Class. Quant. Grav. 8, L89 (1991).

[12] R. R. Metsaev, Mod. Phys. Lett. A 8, 2413 (1993).

[13] R. R. Metsaev, Class. Quant. Grav. 10, L39 (1993).

[14] R. R. Metsaev, Phys. Lett. B 309, 39 (1993).

[15] R. R. Metsaev, Nucl. Phys. B 563, 295 (1999) [arXiv:hep-th/9906217].

[16] R. C. Brower, C. I. Tan and C. B. Thorn, Phys. Rev. D 73, 124037 (2006) [arXiv:hep-th/0603256].

S. J. Brodsky and G. F. de Teramond, arXiv:0709.2072 [hep-ph]; arXiv:0707.3859 [hep-ph].

[17] B. de Wit, J. Hoppe and H. Nicolai, Nucl. Phys. B 305, 545 (1988).

E. Bergshoeff, E. Sezgin, Y. Tanii and P. K. Townsend, Annals Phys. 199, 340 (1990).

[18] O. Bergman and C. B. Thorn, Phys. Rev. D 52, 5980 (1995) [arXiv:hep-th/9506125].

[19] E. S. Fradkin and M. A. Vasiliev, Phys. Lett. B 189, 89 (1987).

[20] M. A. Vasiliev, Phys. Lett. B 243, 378 (1990).

M. A. Vasiliev, Phys. Lett. B 567, 139 (2003) [arXiv:hep-th/0304049].

[21] M. A. Vasiliev, Comptes Rendus Physique 5, 1101 (2004) [arXiv:hep-th/0409260].

X. Bekaert, S. Cnockaert, C. Iazeolla and M. A. Vasiliev, arXiv:hep-th/0503128.

[22] K. B. Alkalaev and M. A. Vasiliev, Nucl. Phys. B 655, 57 (2003) [arXiv:hep-th/0206068].

K. Alkalaev, JHEP 1103, 031 (2011) [arXiv:1011.6109 [hep-th]].

N. Boulanger, E. D. Skvortsov, JHEP 1109, 063 (2011). [arXiv:1107.5028 [hep-th]].

N. Boulanger, E.D. Skvortsov, Y.M. Zinoviev, J.Phys. A A44, 415403 (2011). [1107.1872 [hep-th]].

M. Vasilev, arXiv:1108.5921 [hep-th].

E. Joung and M. Taronna, arXiv:1110.5918 [hep-th].

[23] N. Boulanger and P. Sundell, J. Phys. A 44, 495402 (2011) [arXiv:1102.2219 [hep-th]].

[24] M. A. Vasiliev, Annals Phys. 190, 59 (1989).

[25] R. R. Metsaev, Phys. Lett. B 531, 152 (2002) [arXiv:hep-th/0201226]. 
[26] R. R. Metsaev, Phys. Lett. B 590, 95 (2004) [arXiv:hep-th/0312297].

[27] R. R. Metsaev, Nucl. Phys. B 759, 147 (2006) [arXiv:hep-th/0512342].

[28] X. Bekaert, N. Boulanger and S. Cnockaert, JHEP 0601, 052 (2006) [arXiv:hep-th/0508048].

N. Boulanger, S. Leclercq and S. Cnockaert, Phys. Rev. D 73, 065019 (2006) [arXiv:hep-th/0509118].

M. Porrati and R. Rahman, Nucl. Phys. B 814, 370 (2009) [arXiv:0812.4254 [hep-th]].

X. Bekaert, N. Boulanger and S. Leclercq, J. Phys. A 43, 185401 (2010) [arXiv:1002.0289[hep-th]].

A. Sagnotti and M. Taronna, Nucl. Phys. B 842, 299 (2011) [arXiv:1006.5242 [hep-th]].

R. Manvelyan, K. Mkrtchyan and W. Ruhl, Nucl. Phys. B 826, 1 (2010) [arXiv:0903.0243 [hep-th]]; Phys. Lett. B 696, 410 (2011) [arXiv:1009.1054 [hep-th]].

J. K. Barrett and G. Savvidy, Phys. Lett. B 652, 141 (2007) [arXiv:0704.3164 [hep-th]].

D. Polyakov, Int. J. Mod. Phys. A 25, 4623 (2010); [arXiv:1005.5512 [hep-th]]. Phys. Rev. D 83, 046005 (2011) [arXiv:1011.0353 [hep-th]].

[29] S. Weinberg, Phys.Rev.181, 1893 (1969). Phys.Rev.133, B1318 (1964); Phys.Rev.134, B882 (1964);

F.A.Berends, G.J.H.Burgers and H.Van Dam, Z.Phys.C 24, 247 (1984); Nucl.Phys.B 260, 295 (1985);

Nucl. Phys. B 271, 429 (1986).

I. G. Koh and S. Ouvry, Phys. Lett. B 179, 115 (1986) [Erratum-ibid. 183B, 434 (1987)].

S. Deser and Z. Yang, Class. Quant. Grav. 7, 1491 (1990).

[30] X. Bekaert, N. Boulanger and P. Sundell, arXiv:1007.0435 [hep-th].

[31] J. E. Paton and H. M. Chan, Nucl. Phys. B 10, 516 (1969).

[32] R. R. Metsaev, Mod. Phys. Lett. A 6, 2411 (1991).

[33] M. Goroff and J. H. Schwarz, Phys. Lett. B 127, 61 (1983).

T. Hori, Nucl. Phys. B 274, 401 (1986).

C. Aragone and A. Khoudeir, Class. Quant. Grav. 7, 1291 (1990).

[34] A. A. Tseytlin, Phys. Lett. B 185, 59 (1987).

[35] R. R. Metsaev, Phys. Rev. D 77, 025032 (2008) [arXiv:hep-th/0612279].

R. R. Metsaev, Phys. Lett. B 643, 205 (2006) [arXiv:hep-th/0609029].

[36] R. R. Metsaev and A. A. Tseytlin, Phys. Lett. B 185, 52 (1987).

[37] S. N. Gupta and W. W. Repko, Phys. Rev. 165, 1415 (1968)

S. Ferrara, M. Porrati and V. L. Telegdi, Phys. Rev. D 46, 3529 (1992).

[38] S. M. Klishevich, Int. J. Mod. Phys. A 15, 609 (2000) [arXiv:hep-th/9811030].

[39] S.M.Klishevich and Y.M.Zinovev, Phys.Atom.Nucl. 61, 1527 (1998); hep-th/9708150.

[40] P. C. Argyres and C. R. Nappi, Phys. Lett. B 224, 89 (1989).

S. M. Klishevich, Int. J. Mod. Phys. A 15, 395 (2000) [arXiv:hep-th/9805174].

[41] A. Cucchieri, M. Porrati and S. Deser, Phys. Rev. D 51, 4543 (1995) [arXiv:hep-th/9408073].

[42] I.L.Buchbinder, D.Gitman, V.Krykhtin, V.Pershin, Nucl.Phys.B 584, 615 (2000) [hep-th/9910188].

I. L. Buchbinder, D. M. Gitman and V. D. Pershin, Phys. Lett. B 492, 161 (2000) hep-th/0006144

[43] A.Fotopoulos, N.Irges, A.C.Petkou and M.Tsulaia, JHEP 0710, 021 (2007) [arXiv:0708.1399]. 